\title{
Mild and Expeditious Synthesis of Sulfenyl Enaminones of L-a- Amino Esters and Aryl/Alkyl Amines through NCS-Mediated Sulfenylation
}

Sayan Mukherjee and Animesh Pramanik*

Department of Chemistry, University of Calcutta, 92, A. P. C. Road, Kolkata 700009, India

Fax: +91-33-2351-9755; Tel: +919830107470

E-mail: pramanikanimesh61@gmail.com

\section{$\underline{\text { Supporting Information }}$}

\begin{tabular}{cc}
\hline Contents & Page No. \\
\hline $\begin{array}{c}\text { NMR spectra of L- } \alpha \text {-amino ester embedded } \\
\text { sulfenyl enaminone derivatives 5 (Figure S1- } \\
\text { S58) }\end{array}$ & S2-S30 \\
NMR spectra of L- $\alpha$-amino ester embedded \\
Selenyl enaminone derivatives 7 (Figure S59- \\
$\begin{array}{c}\text { NMR spectra of } \beta \text {-amino sulfide/selenide } \\
\text { derivatives 9 (Figure S67-S88) }\end{array}$ \\
$\begin{array}{c}\text { NMR spectra of isolated intermediates for } \\
\text { mechanistic study (Figure S89-S96) }\end{array}$ \\
$\begin{array}{c}\text { LC-MS spectra of intermediate I (Figure S97- } \\
\text { S98) }\end{array}$ \\
\hline
\end{tabular}




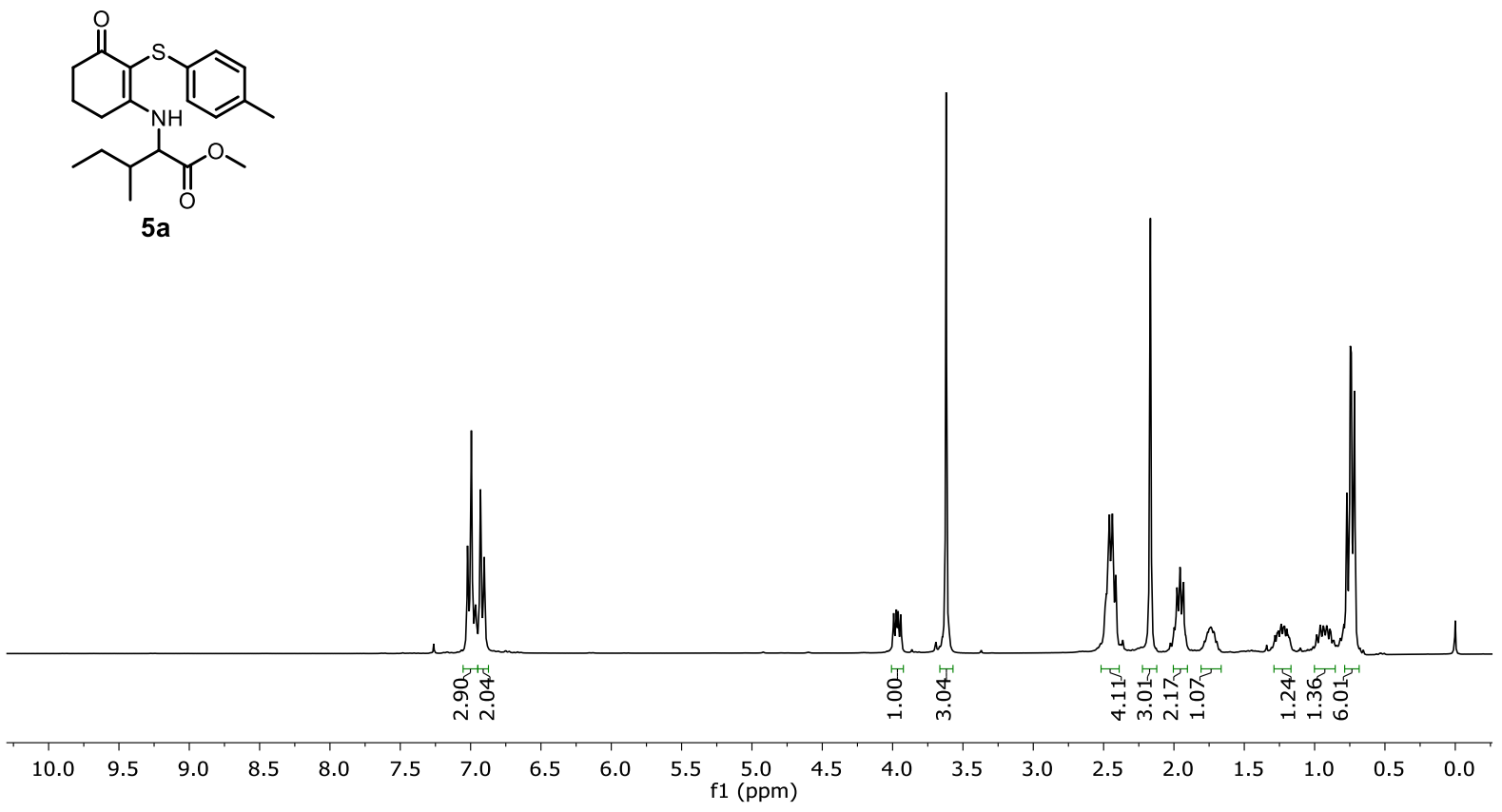

Figure S1: ${ }^{1} \mathrm{H}$ NMR spectrum of compound 5a in $\mathrm{CDCl}_{3}(300 \mathrm{MHz})$

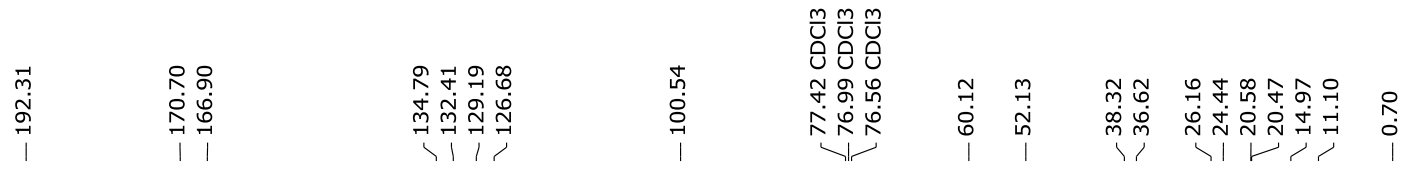<smiles>CCC(C)C(NC1=C(Sc2ccc(C)cc2)C(=O)CCC1)C(=O)OC</smiles>

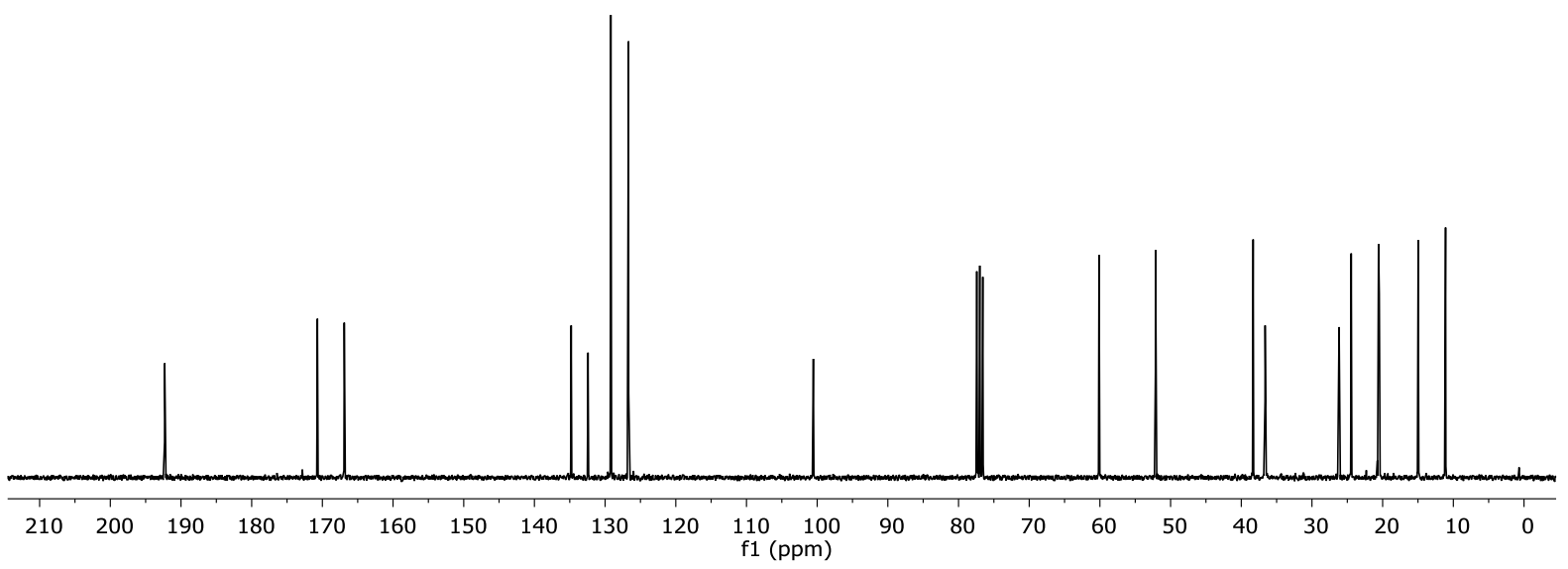

Figure S2: ${ }^{13} \mathrm{C}\left\{{ }^{1} \mathrm{H}\right\}$ NMR spectrum of compound 5a in $\mathrm{CDCl}_{3}(75 \mathrm{MHz})$ 
ญั

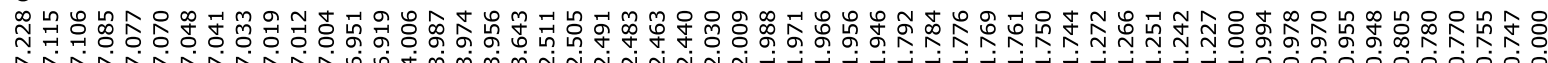

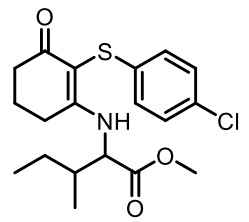

$5 b$

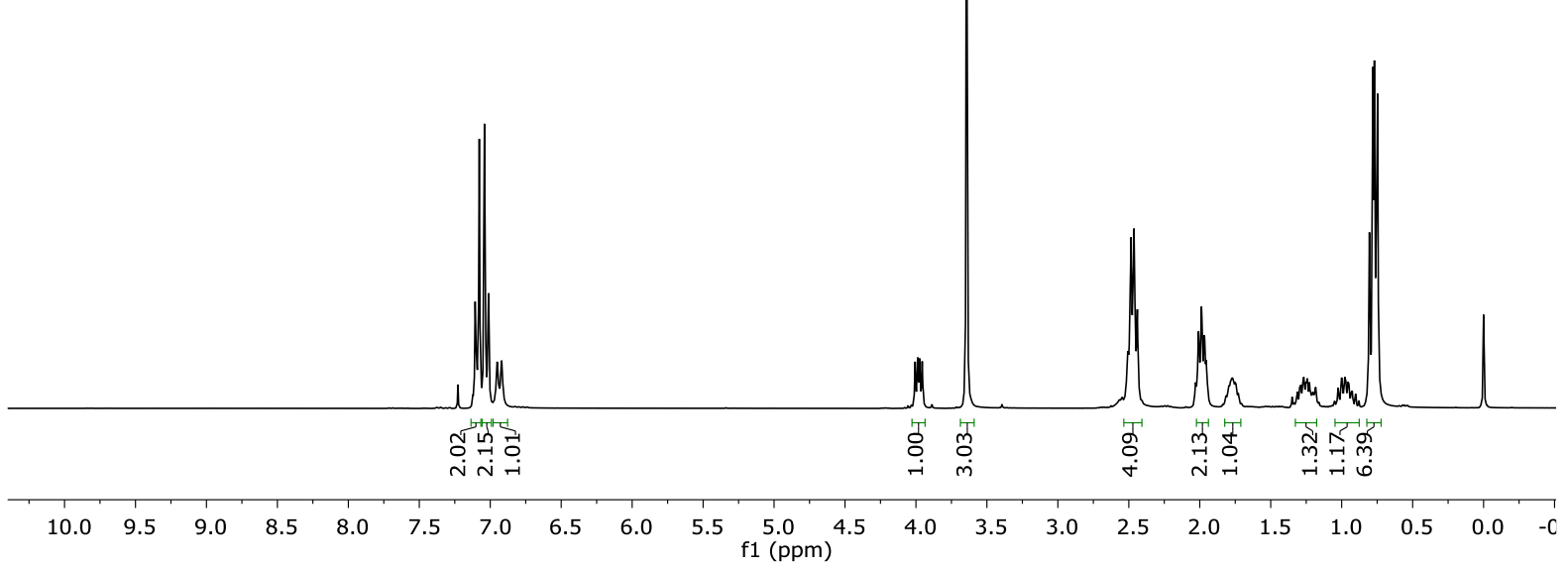

Figure S3: ${ }^{1} \mathrm{H}$ NMR spectrum of compound $\mathbf{5 b}$ in $\mathrm{CDCl}_{3}(300 \mathrm{MHz})$

\begin{tabular}{|c|c|c|c|c|c|c|c|}
\hline 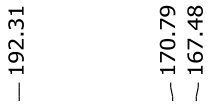 & 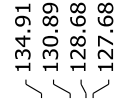 & $\begin{array}{l}\stackrel{0}{2} \\
\dot{\alpha} \\
1\end{array}$ & 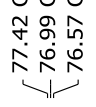 & $\begin{array}{l}\text { Nิ } \\
\stackrel{0}{0} \\
1\end{array}$ & $\underset{\substack{\mathfrak{Z} \\
\text { กิ } \\
1}}{1}$ & 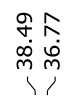 & 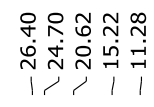 \\
\hline
\end{tabular}<smiles>CCC(C)C(NC1=C(Sc2ccc(Cl)cc2)C(=O)CCC1)C(=O)OC</smiles>

$5 \mathbf{b}$

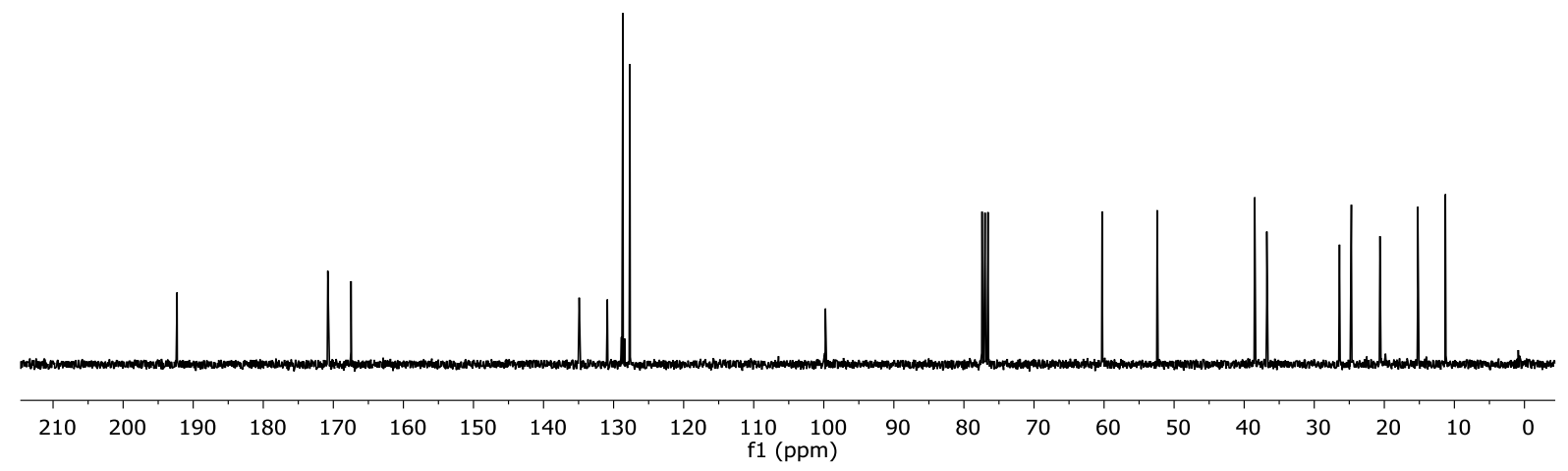

Figure S4: ${ }^{13} \mathrm{C}\left\{{ }^{1} \mathrm{H}\right\}$ NMR spectrum of compound $\mathbf{5 b}$ in $\mathrm{CDCl}_{3}(75 \mathrm{MHz})$ 
<smiles>CCC(C)C(NC1=C(Sc2c(Br)cccc2Br)C(=O)CCC1)C(=O)OC</smiles>

$5 c$

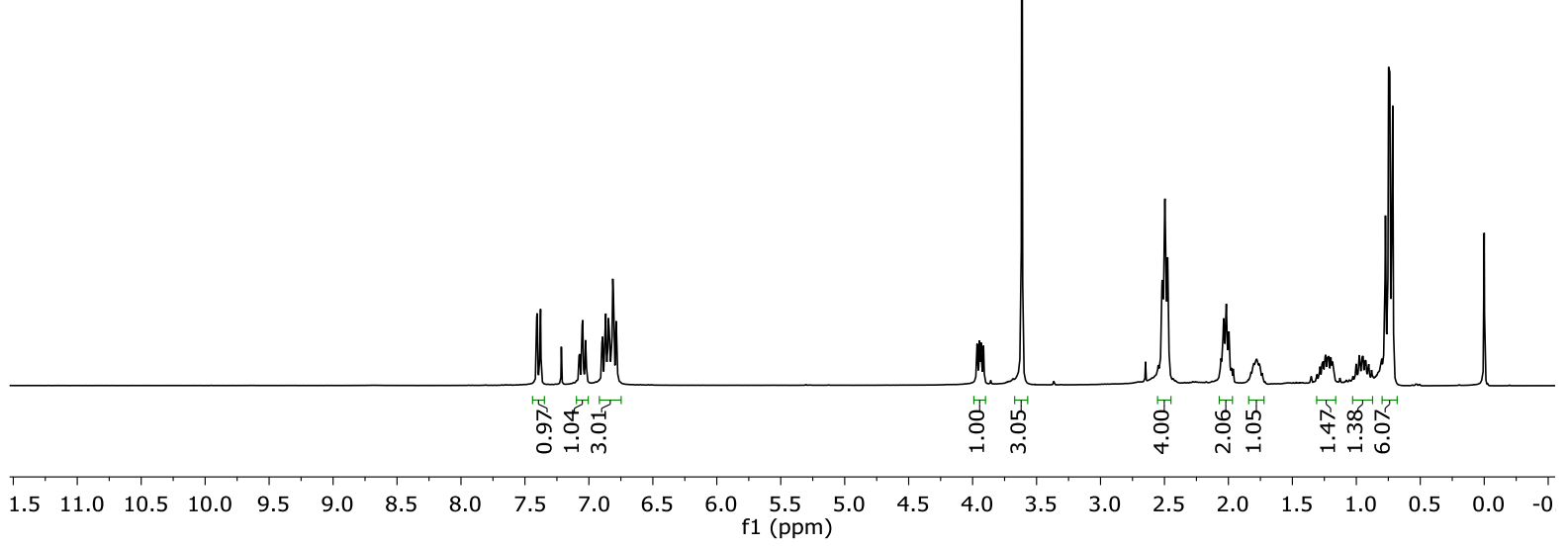

Figure S5: ${ }^{1} \mathrm{H}$ NMR spectrum of compound $\mathbf{5 c}$ in $\mathrm{CDCl}_{3}(300 \mathrm{MHz})$

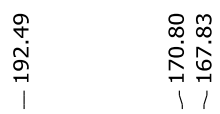

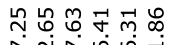<smiles>CCC(C)C(NC1=C(Sc2ccccc2Br)C(=O)CCC1)C(=O)OC</smiles>

5c

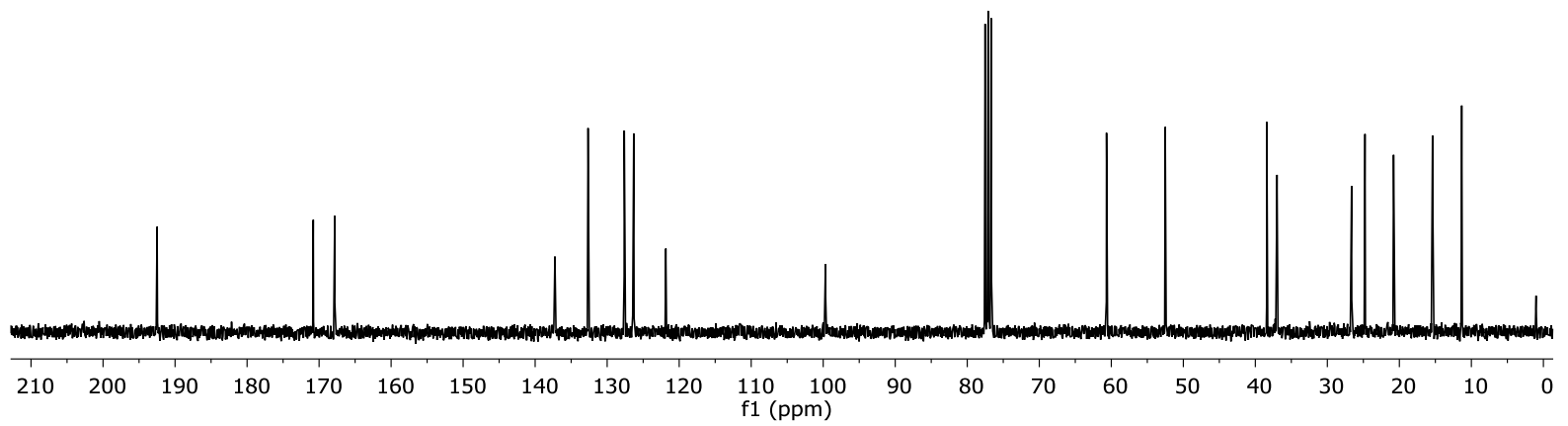

Figure S6: ${ }^{13} \mathrm{C}\left\{{ }^{1} \mathrm{H}\right\}$ NMR spectrum of compound $\mathbf{5 c}$ in $\mathrm{CDCl}_{3}(75 \mathrm{MHz})$ 
<smiles>CCC(C)C(NC1=C(Sc2ccc(C)cc2)C(=O)CC(C)(C)C1)C(=O)OC</smiles>

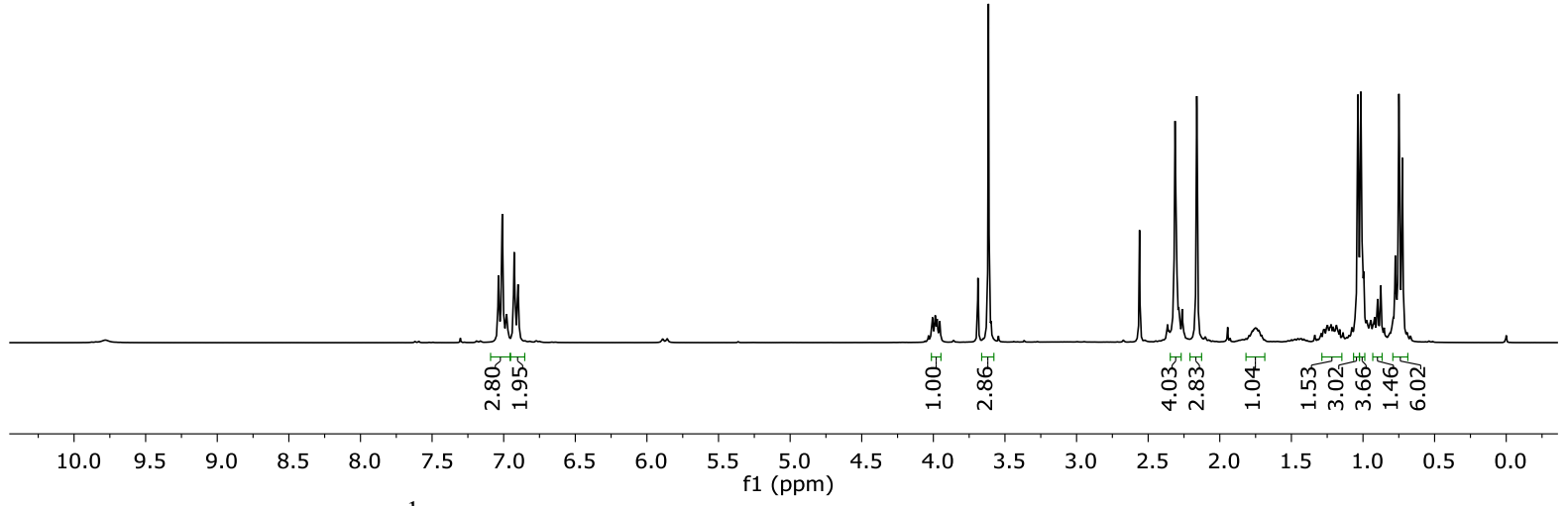

Figure S7: ${ }^{1} \mathrm{H}$ NMR spectrum of compound $\mathbf{5 d}$ in $\mathrm{CDCl}_{3}(300 \mathrm{MHz})$<smiles>CCC(C)C(NC1=C(Sc2ccc(C)cc2)C(=O)CC(C)(C)C1)C(=O)OC</smiles>

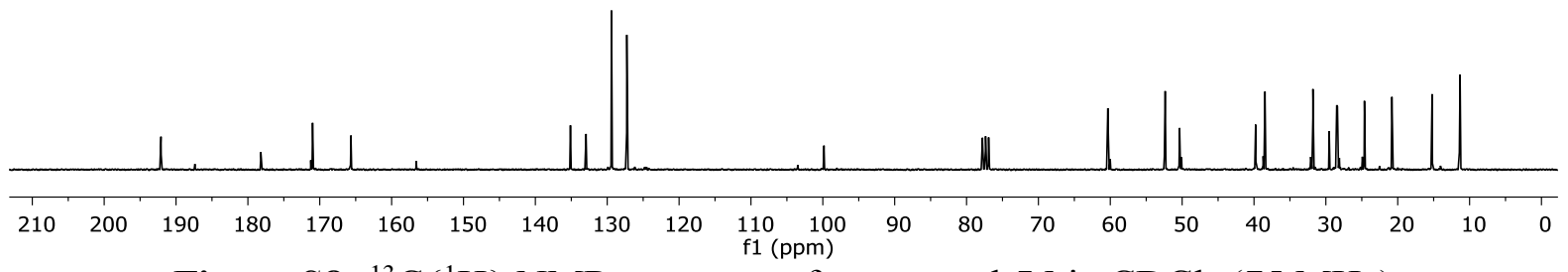

Figure S8: ${ }^{13} \mathrm{C}\left\{{ }^{1} \mathrm{H}\right\}$ NMR spectrum of compound 5d in $\mathrm{CDCl}_{3}(75 \mathrm{MHz})$ 
<smiles>CCC(C)C(NC1=C(Sc2ccc(F)cc2)C(=O)CC(C)(C)C1)C(=O)O</smiles>

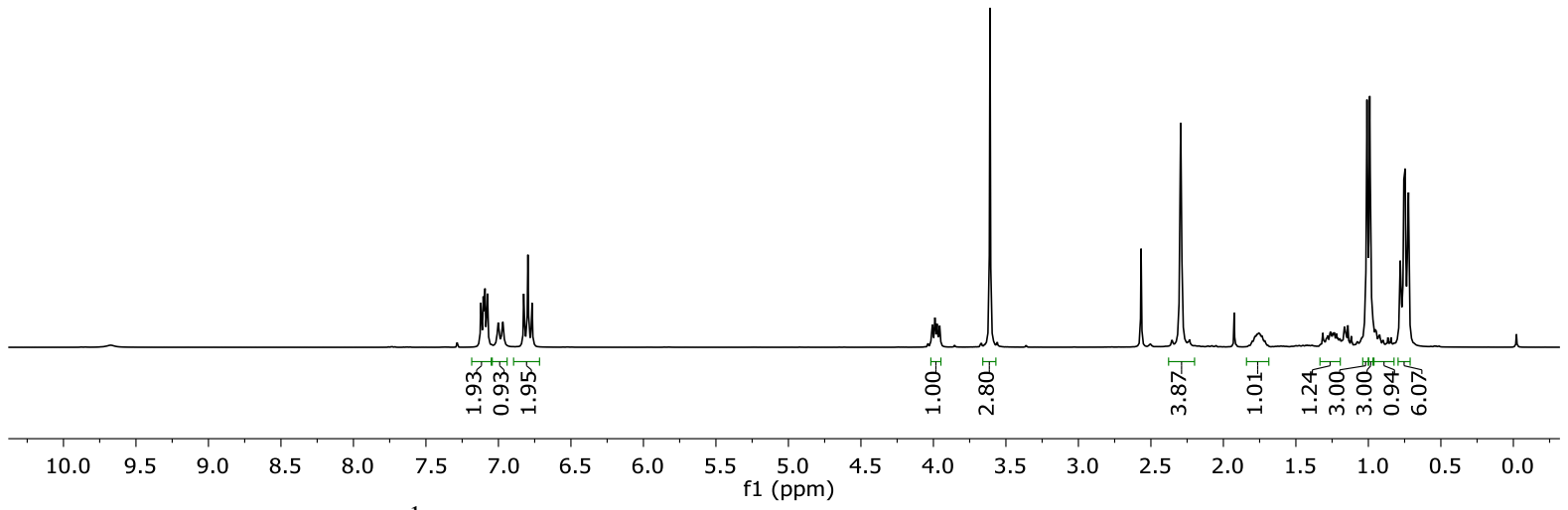

Figure S9: ${ }^{1} \mathrm{H}$ NMR spectrum of compound $5 \mathbf{e}$ in $\mathrm{CDCl}_{3}(300 \mathrm{MHz})$<smiles>CCC(C)C(=O)C(Nc1ccc(F)cc1)Sc1ccc(F)cc1</smiles>

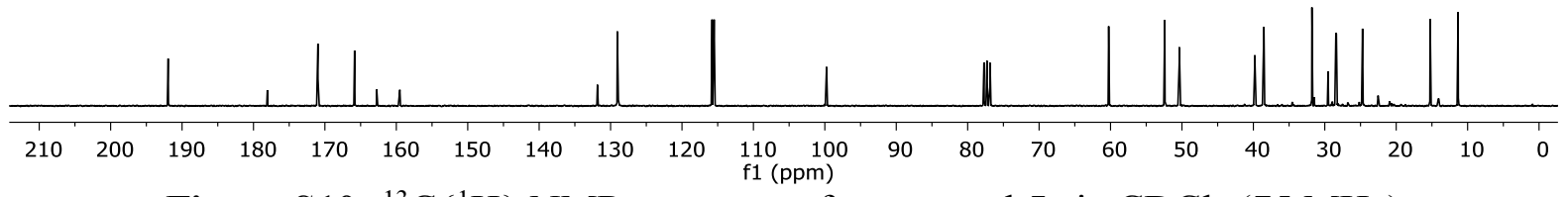

Figure S10: ${ }^{13} \mathrm{C}\left\{{ }^{1} \mathrm{H}\right\}$ NMR spectrum of compound $5 \mathbf{e}$ in $\mathrm{CDCl}_{3}(75 \mathrm{MHz})$ 
商<smiles>COC(=O)C(Cc1ccccc1)NC1=C(Sc2ccccc2)C(=O)CC(C)(C)C1</smiles>

Figure S11: ${ }^{1} \mathrm{H}$ NMR spectrum of compound $\mathbf{5} f$ in $\mathrm{CDCl}_{3}(500 \mathrm{MHz})$<smiles>COC(=O)C(Cc1ccccc1)NC1=C(Sc2ccccc2)C(=O)CC(C)(C)C1</smiles>

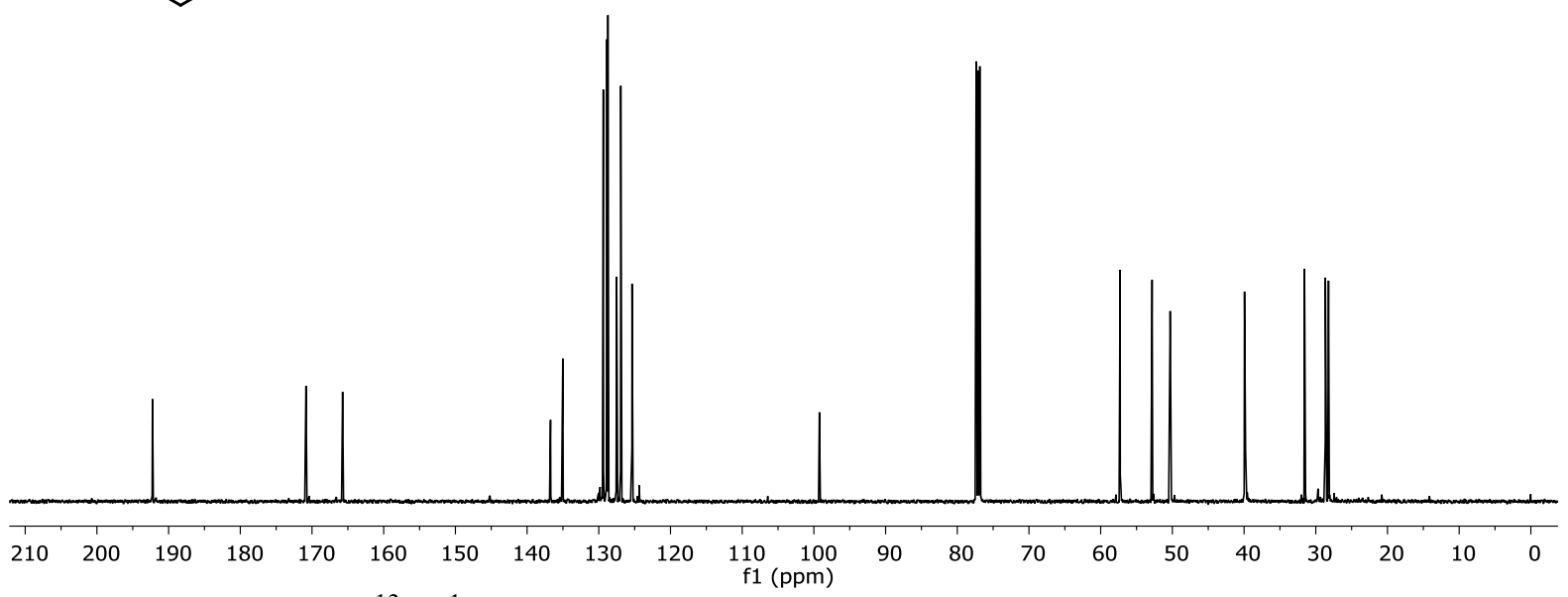

Figure S12: ${ }^{13} \mathrm{C}\left\{{ }^{1} \mathrm{H}\right\}$ NMR spectrum of compound $\mathbf{5 f}$ in $\mathrm{CDCl}_{3}(126 \mathrm{MHz})$ 
<smiles>COC(=O)C(Cc1ccc[13c]c1)NC1=C(Sc2ccc(C)cc2)C(=O)CC(C)(C)C1</smiles>

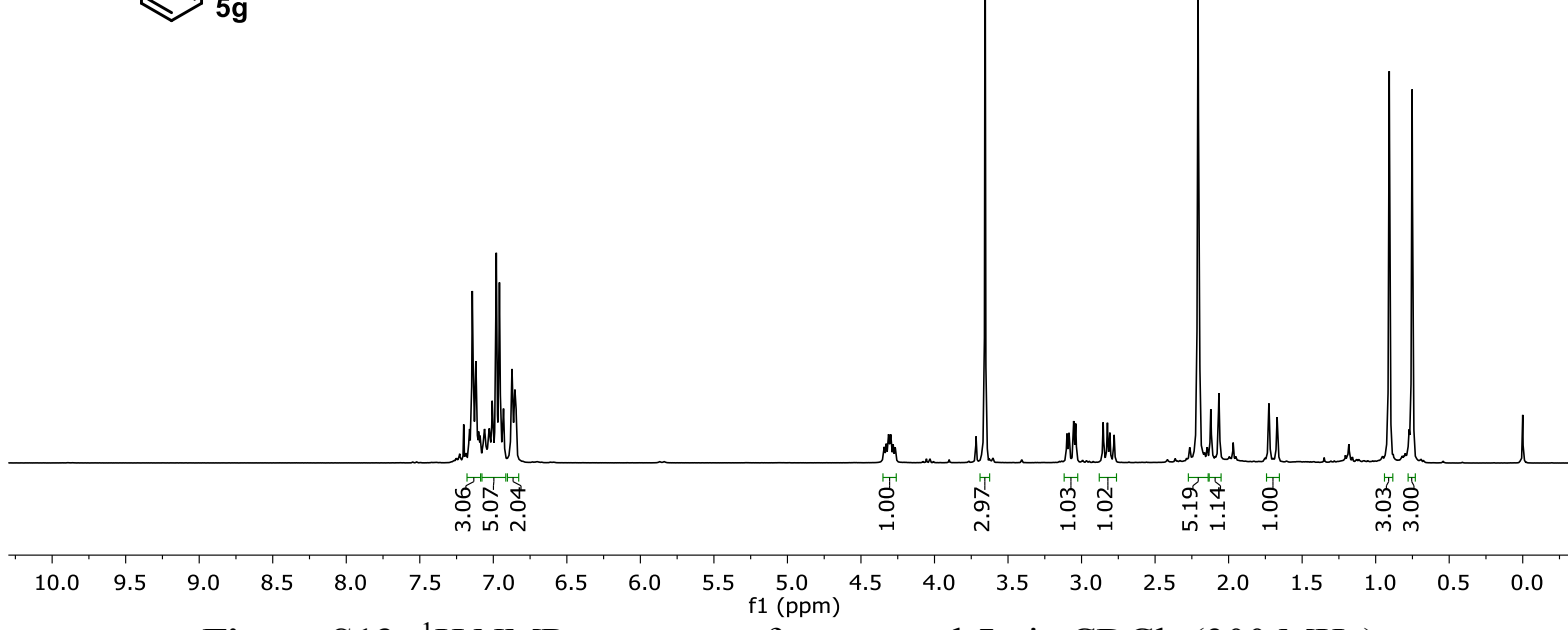

Figure S13: ${ }^{1} \mathrm{H}$ NMR spectrum of compound $\mathbf{5 g}$ in $\mathrm{CDCl}_{3}(300 \mathrm{MHz})$<smiles>COC(=O)C(Cc1ccc[13c]c1)NC1=C(Sc2ccc(C)cc2)C(=O)CC(C)(C)C1</smiles>

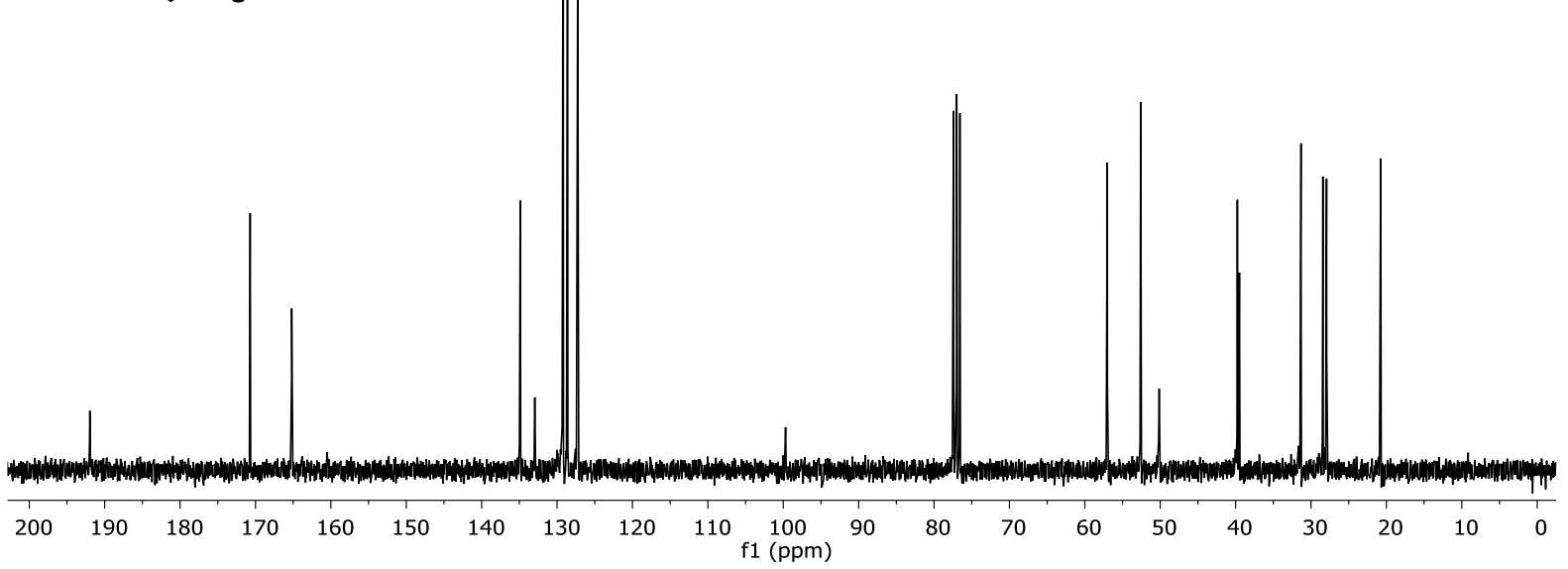

Figure S14: ${ }^{13} \mathrm{C}\left\{{ }^{1} \mathrm{H}\right\}$ NMR spectrum of compound $\mathbf{5 g}$ in $\mathrm{CDCl}_{3}(75 \mathrm{MHz})$ 
<smiles>COC(=O)C(Cc1ccc[se]1)NC1=C(Sc2ccc(Cl)cc2)C(=O)CC(C)(C)C1</smiles>

$5 \mathrm{~h}$

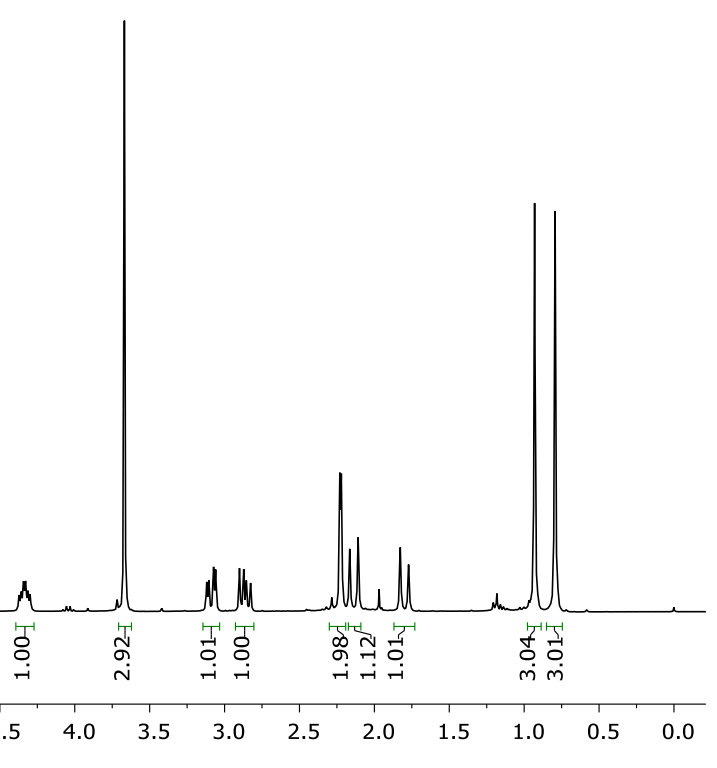

Figure S15: ${ }^{1} \mathrm{H}$ NMR spectrum of compound $\mathbf{5 h}$ in $\mathrm{CDCl}_{3}(300 \mathrm{MHz})$<smiles></smiles>

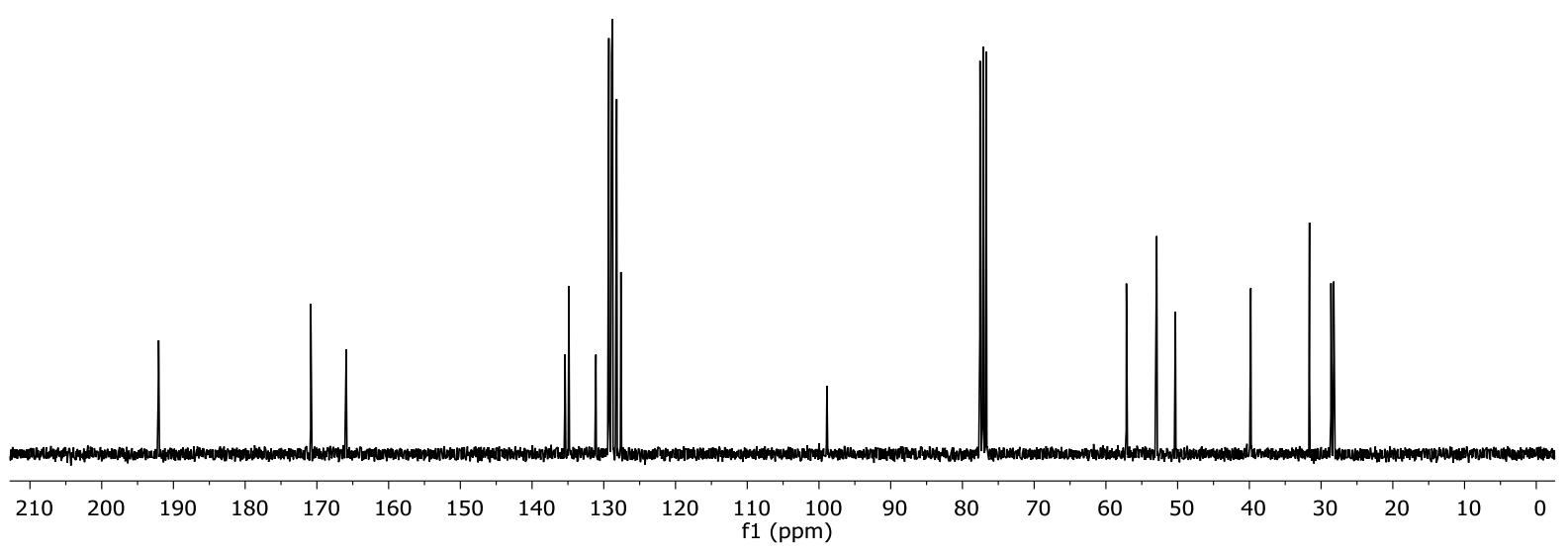

Figure S16: ${ }^{13} \mathrm{C}\left\{{ }^{1} \mathrm{H}\right\}$ NMR spectrum of compound $\mathbf{5 h}$ in $\mathrm{CDCl}_{3}(75 \mathrm{MHz})$ 
ֻั

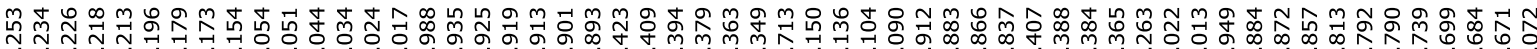

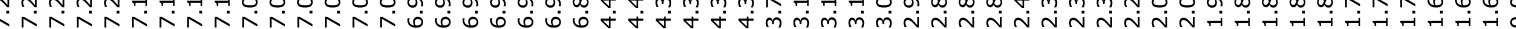<smiles>COC(=O)C(Cc1ccccc1)NC1=C(Sc2ccc(C)cc2)C(=O)CCC1</smiles>

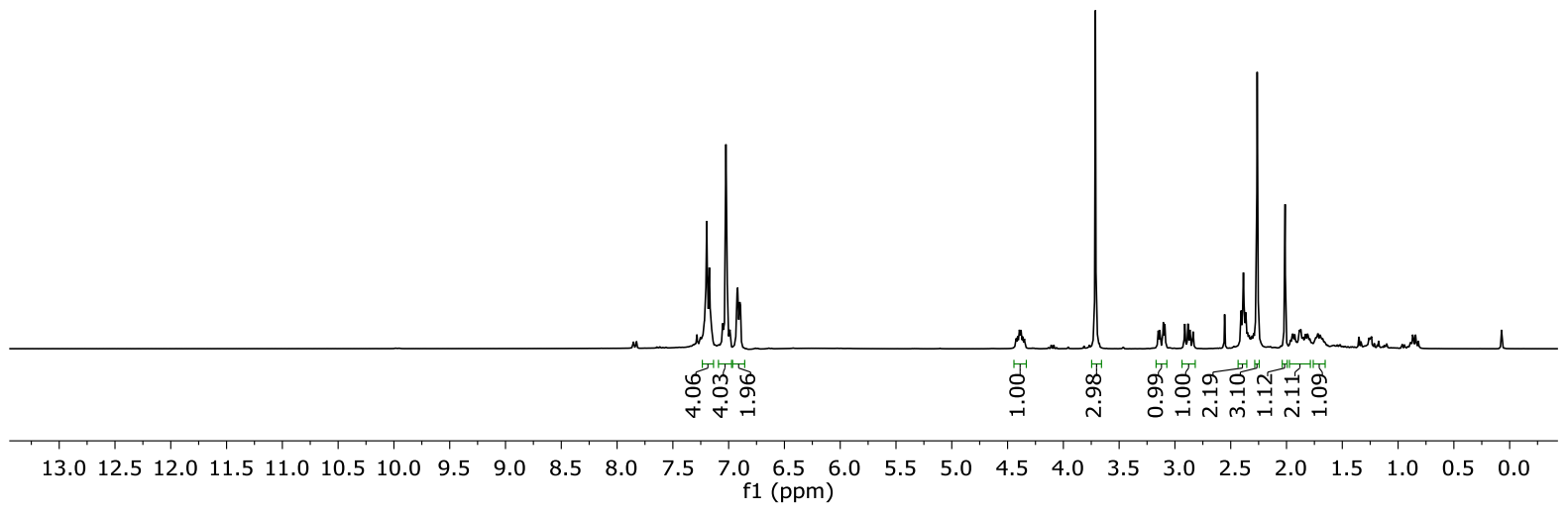

Figure S17: ${ }^{1} \mathrm{H}$ NMR spectrum of compound $\mathbf{5 i}$ in $\mathrm{CDCl}_{3}(300 \mathrm{MHz})$<smiles>COC(=O)C(Cc1ccccc1)Nc1ccc(C)cc1Sc1ccccc1</smiles>

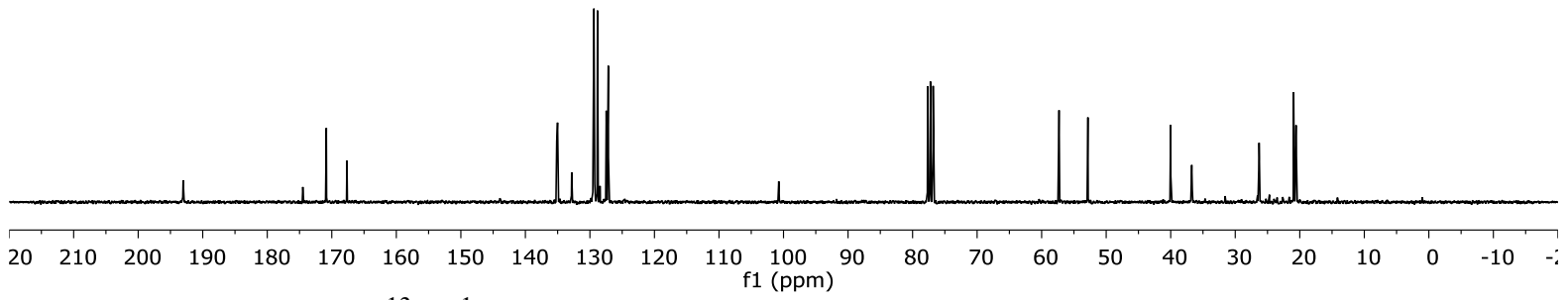

Figure S18: ${ }^{13} \mathrm{C}\left\{{ }^{1} \mathrm{H}\right\}$ NMR spectrum of compound $5 \mathbf{i}$ in $\mathrm{CDCl}_{3}(75 \mathrm{MHz})$ 


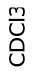

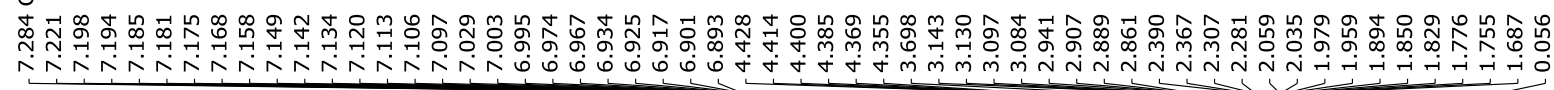<smiles></smiles>

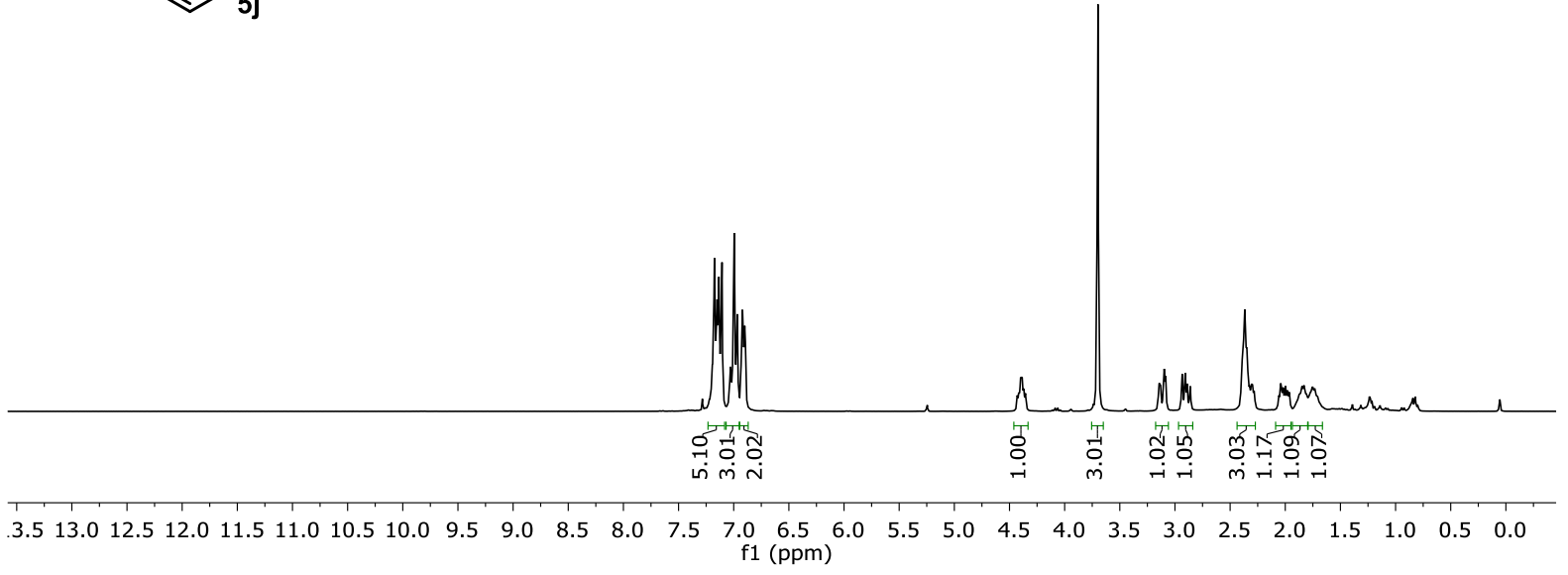

Figure S19: ${ }^{1} \mathrm{H}$ NMR spectrum of compound $\mathbf{5 j}$ in $\mathrm{CDCl}_{3}(300 \mathrm{MHz})$

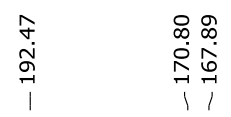<smiles></smiles>

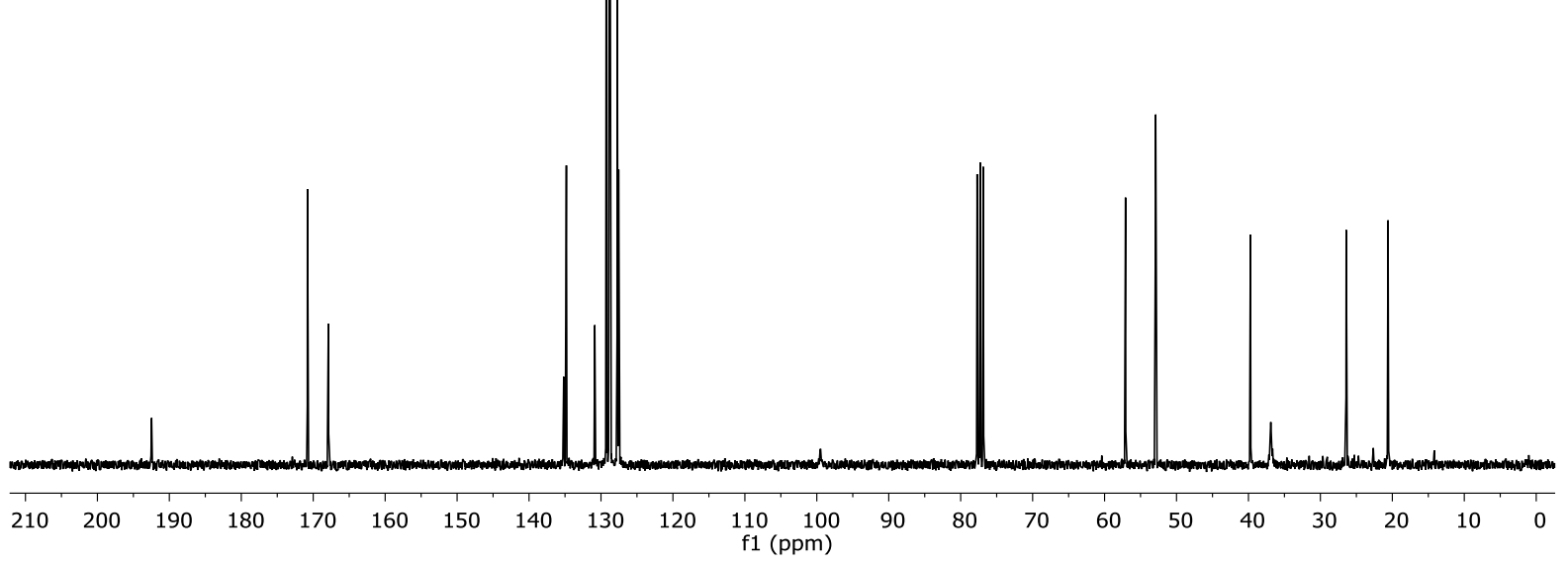

Figure S20: ${ }^{13} \mathrm{C}\left\{{ }^{1} \mathrm{H}\right\}$ NMR spectrum of compound $\mathbf{5 j}$ in $\mathrm{CDCl}_{3}(75 \mathrm{MHz})$ 


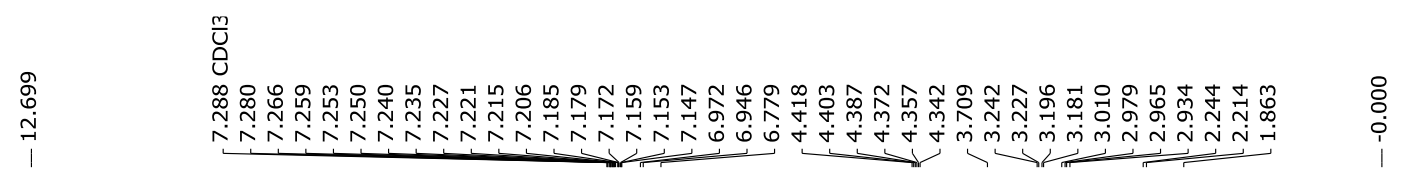<smiles>COC(=O)C(Cc1ccccc1)NC(C(=O)O)=C(Sc1ccc(C)cc1)C(C)=O</smiles>

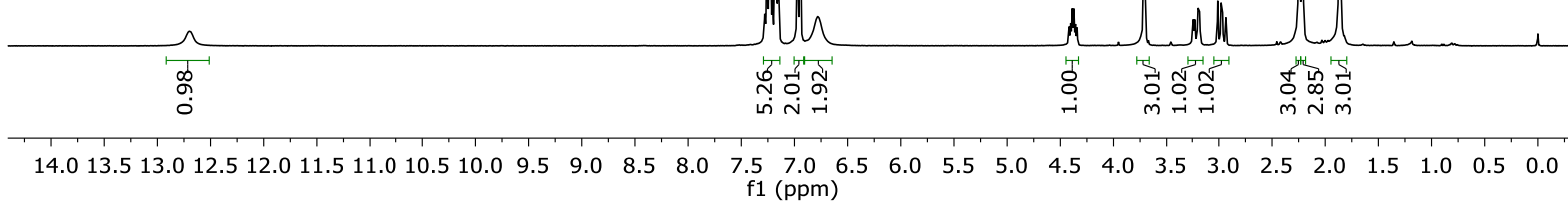

Figure S21: ${ }^{1} \mathrm{H}$ NMR spectrum of compound 5k in $\mathrm{CDCl}_{3}(300 \mathrm{MHz})$

ก̂ก

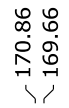

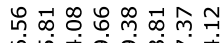

弚

$m$
$\overline{\mathrm{U}}$
$\mathrm{O}$
$\mathrm{O}$

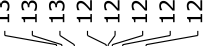

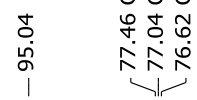

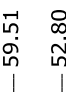

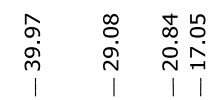<smiles>COC(=O)C(Cc1ccccc1)N/C(C)=C(/Sc1ccc(C)cc1)C(C)=O</smiles>

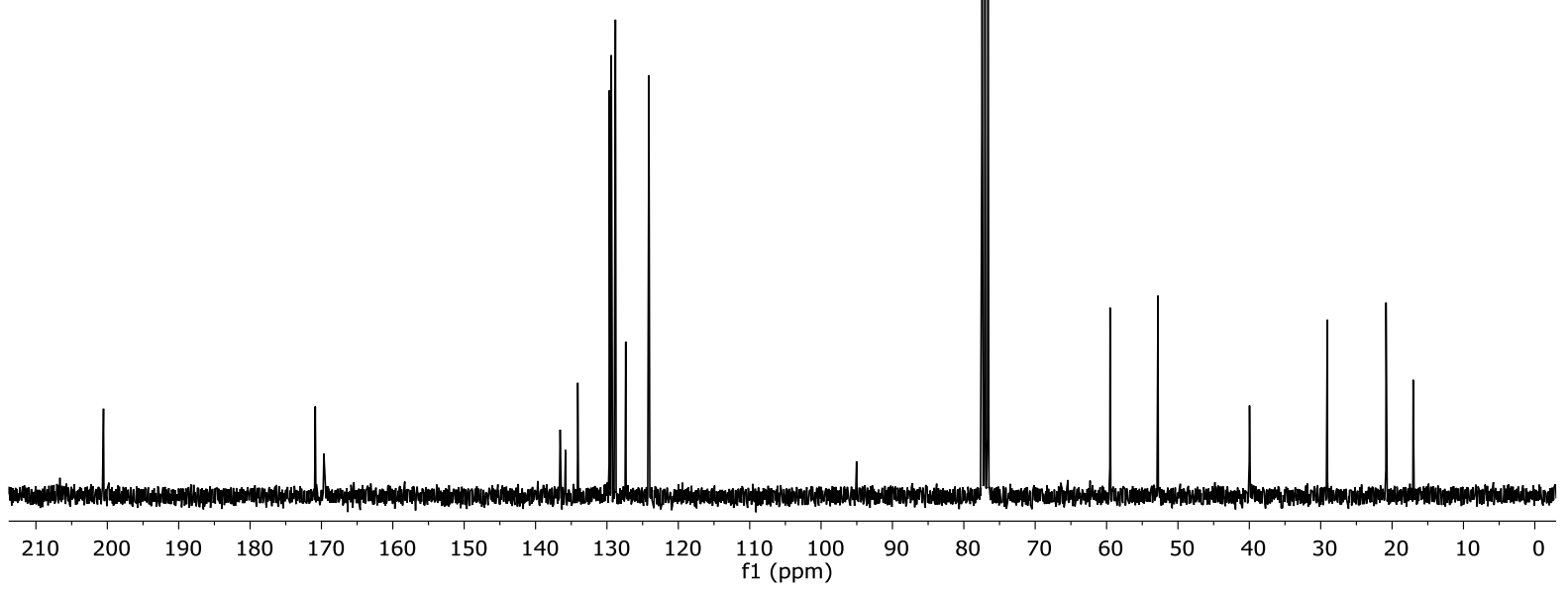

Figure S22: ${ }^{13} \mathrm{C}\left\{{ }^{1} \mathrm{H}\right\}$ NMR spectrum of compound 5k in $\mathrm{CDCl}_{3}(75 \mathrm{MHz})$ 


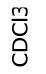

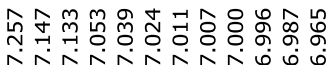

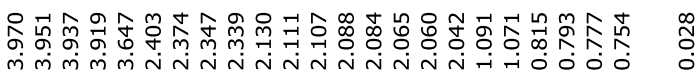

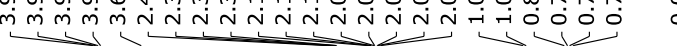<smiles>COC(=O)C(NC1=C(Sc2ccccc2)C(=O)CC(C)(C)C1)C(C)C</smiles>

5 I

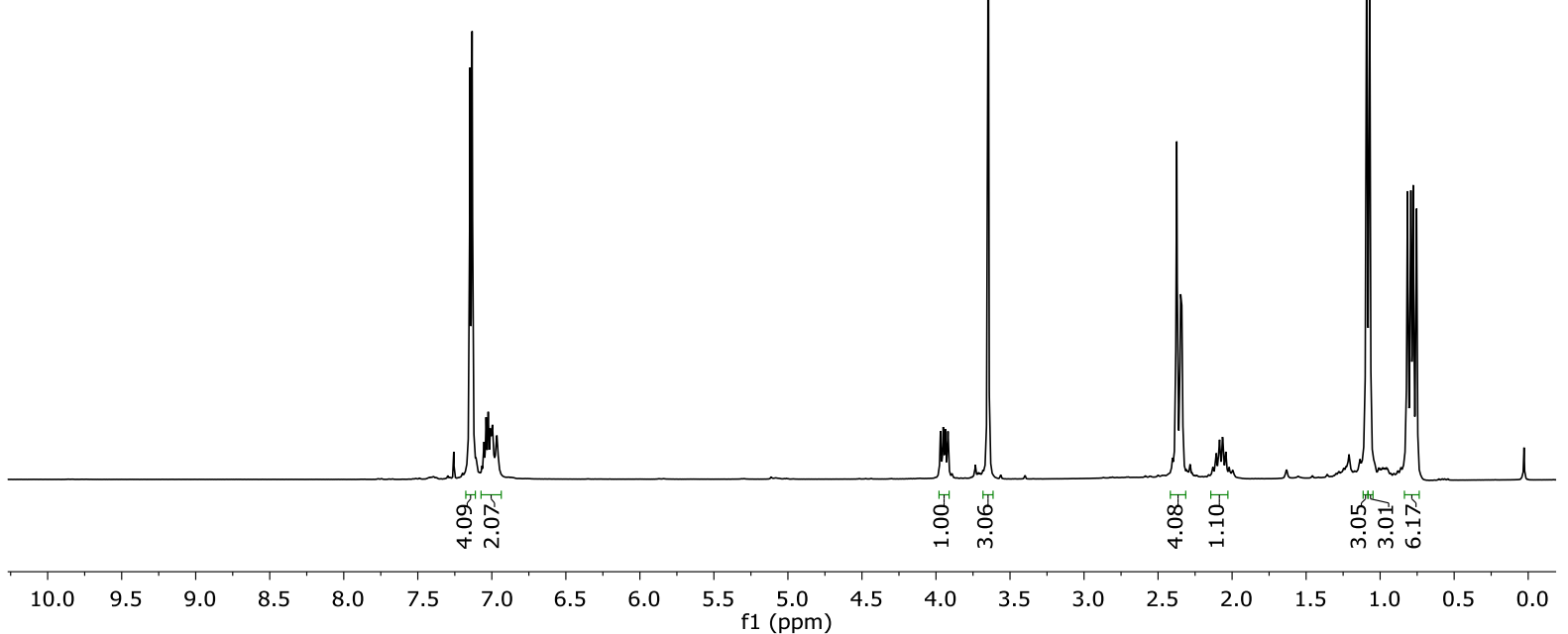

Figure S23: ${ }^{1} \mathrm{H}$ NMR spectrum of compound $\mathbf{5 l}$ in $\mathrm{CDCl}_{3}(300 \mathrm{MHz})$

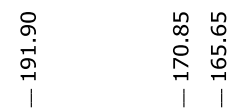

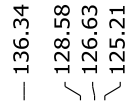

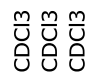

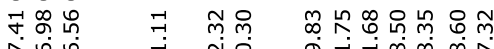

송

$\dot{m} \dot{m} \stackrel{\infty}{N} \stackrel{\infty}{\sim}$<smiles>COC(=O)C(NC1=CC(=O)CC(C)(C)C1)C(C)C</smiles>

$5 \mathbf{1}$

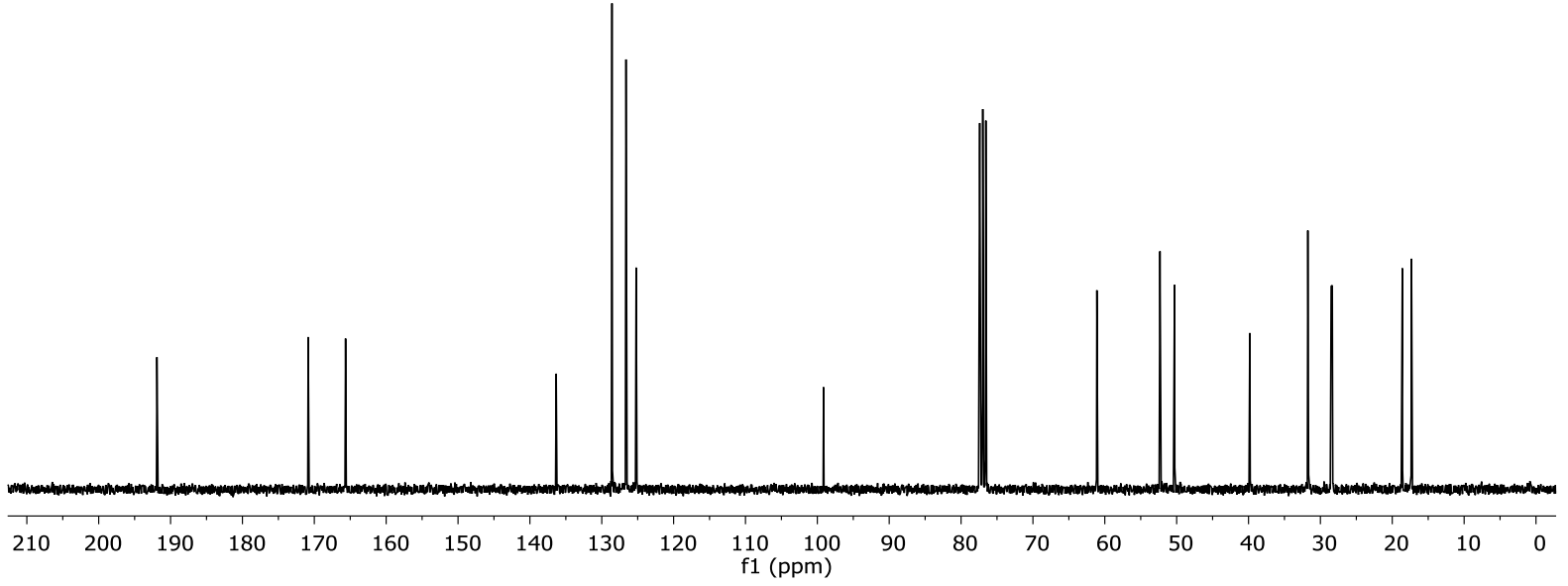

Figure S24: ${ }^{13} \mathrm{C}\left\{{ }^{1} \mathrm{H}\right\}$ NMR spectrum of compound $\mathbf{5 l}$ in $\mathrm{CDCl}_{3}(75 \mathrm{MHz})$ 

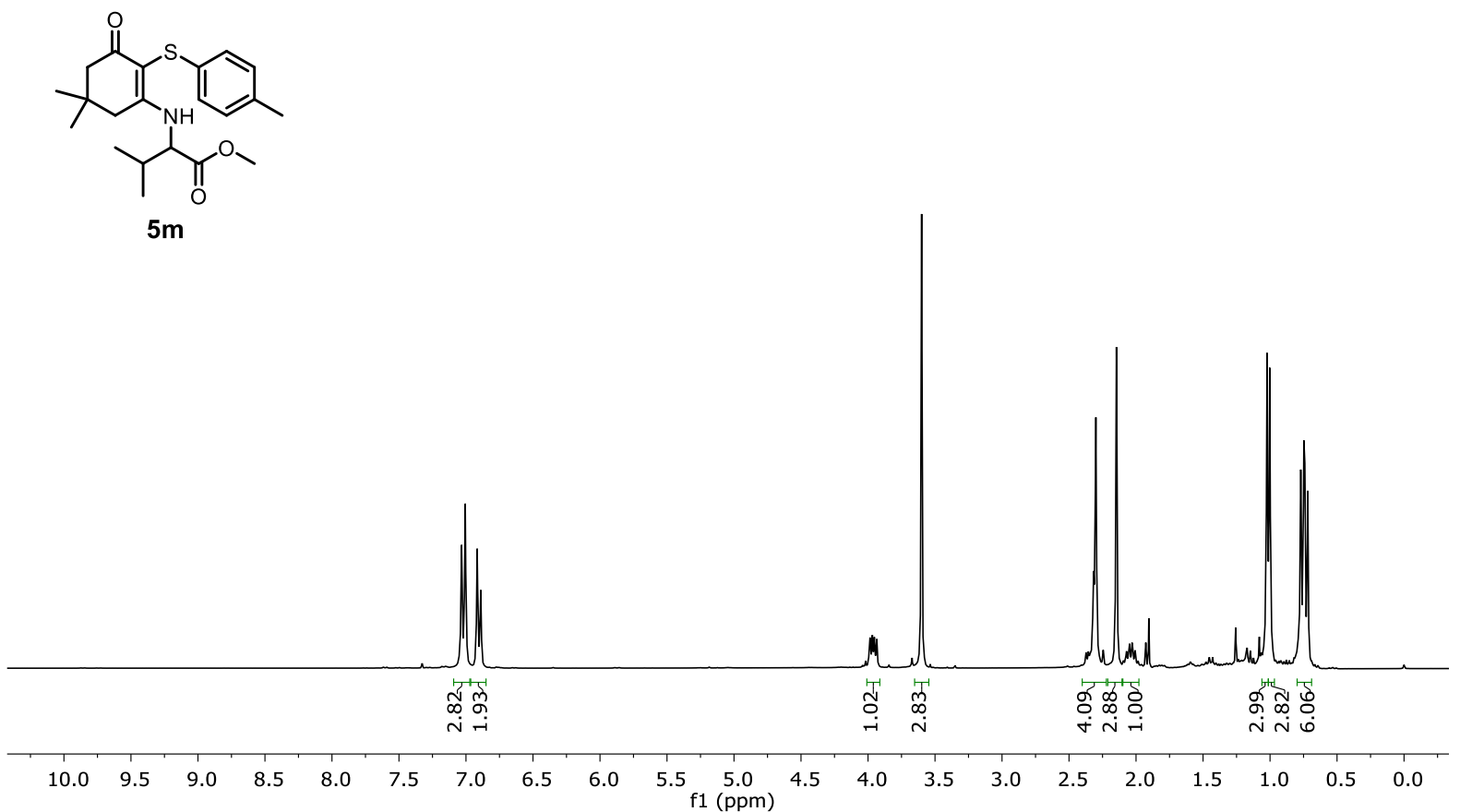

Figure S25: ${ }^{1} \mathrm{H}$ NMR spectrum of compound $\mathbf{5 m}$ in $\mathrm{CDCl}_{3}(300 \mathrm{MHz})$<smiles>COC(=O)C(NC1=C(Sc2ccc(C)cc2)C(=O)CC(C)(C)C1)C(C)C</smiles>

$5 \mathrm{~m}$

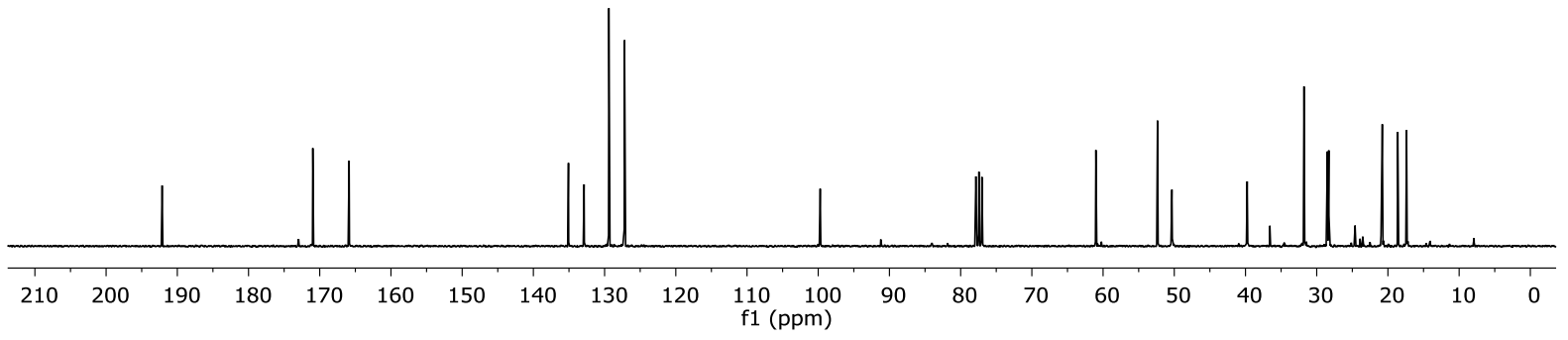

Figure S26: ${ }^{13} \mathrm{C}\left\{{ }^{1} \mathrm{H}\right\}$ NMR spectrum of compound $\mathbf{5 m}$ in $\mathrm{CDCl}_{3}(75 \mathrm{MHz})$ 
రัญ
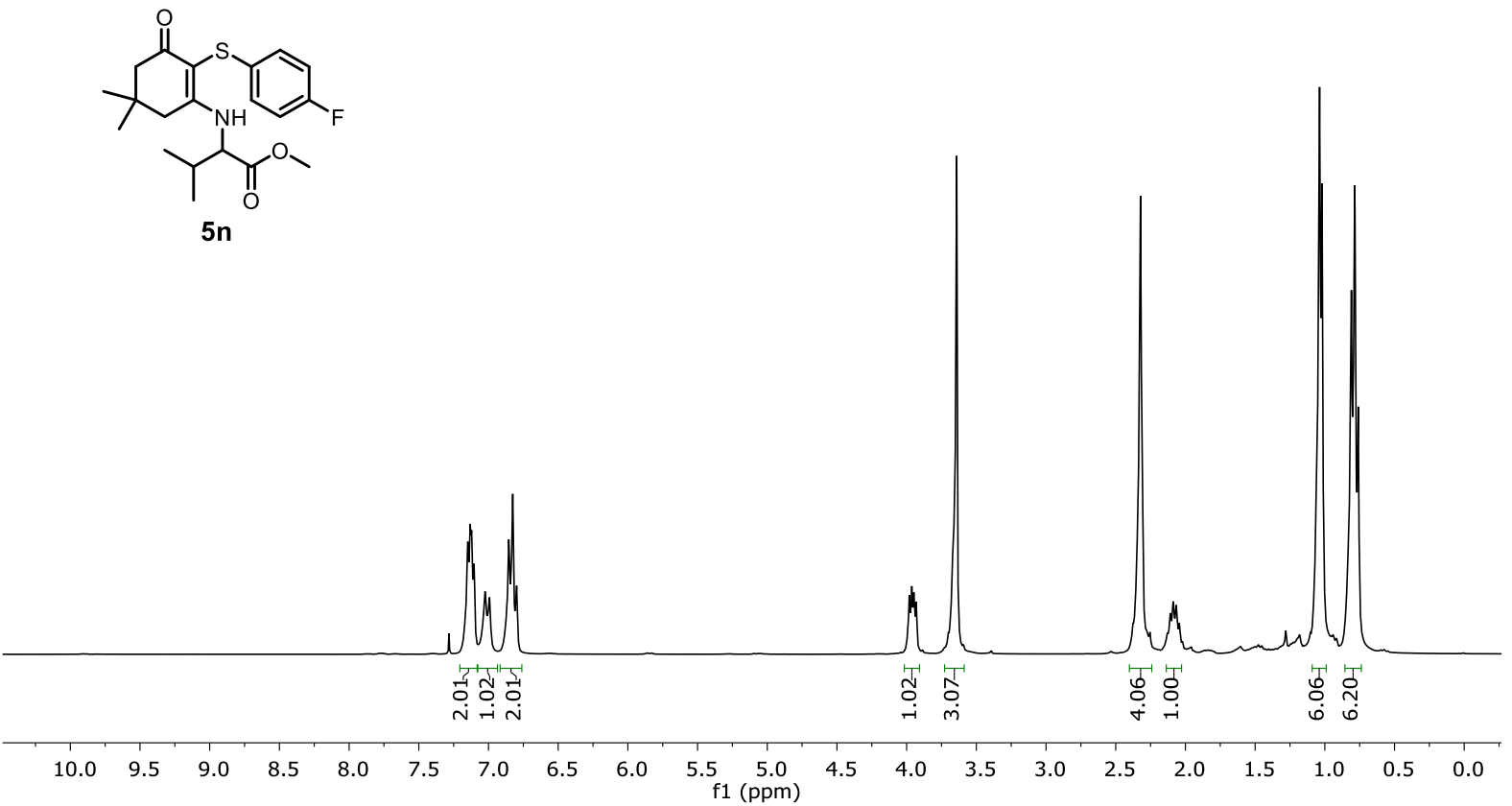

Figure S27: ${ }^{1} \mathrm{H}$ NMR spectrum of compound $\mathbf{5 n}$ in $\mathrm{CDCl}_{3}(300 \mathrm{MHz})$
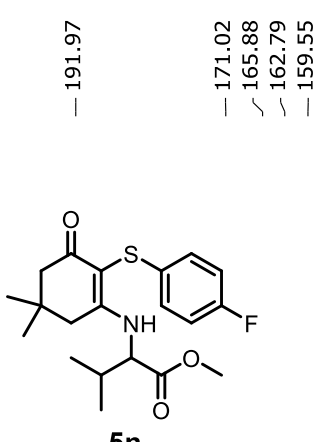

$5 n$

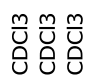

ํำ

송

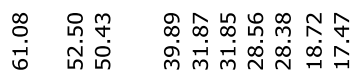

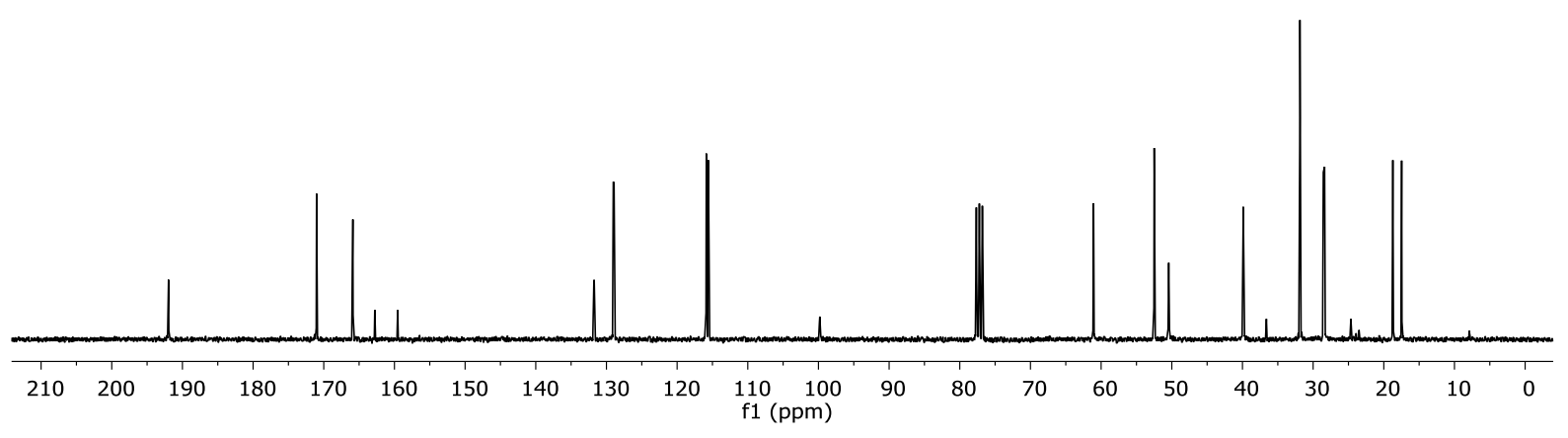

Figure S28: ${ }^{13} \mathrm{C}\left\{{ }^{1} \mathrm{H}\right\}$ NMR spectrum of compound $\mathbf{5 n}$ in $\mathrm{CDCl}_{3}(75 \mathrm{MHz})$ 
啻

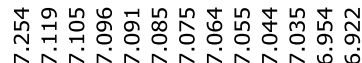

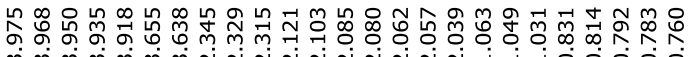

-i<smiles>COC(=O)C(Nc1ccc(Cl)cc1)Sc1ccc(Cl)cc1</smiles>

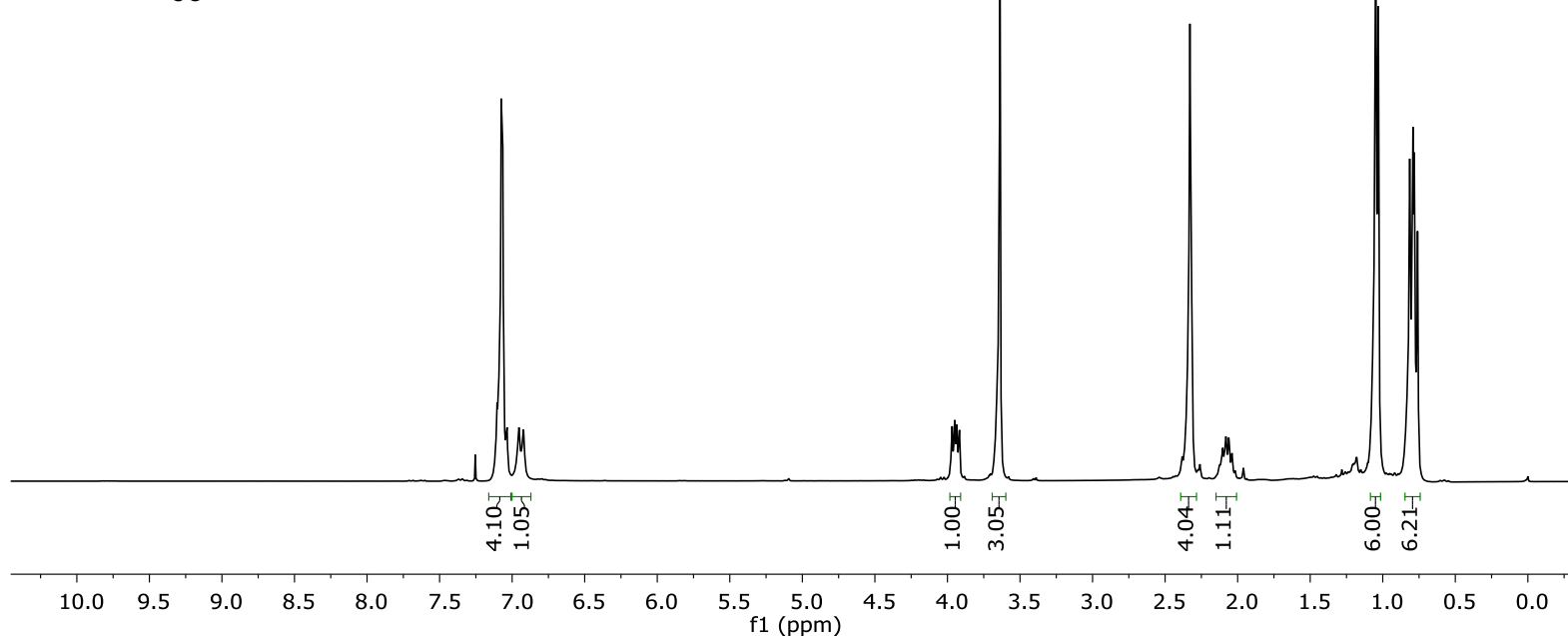

Figure S29: ${ }^{1} \mathrm{H}$ NMR spectrum of compound 50 in $\mathrm{CDCl}_{3}(300 \mathrm{MHz})$

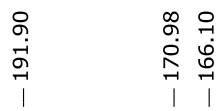

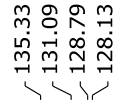

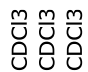

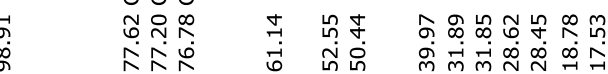<smiles>COC(=O)C(NC1=C(Sc2ccc(Cl)cc2)C(=O)CC(C)(C)C1)C(C)C</smiles>

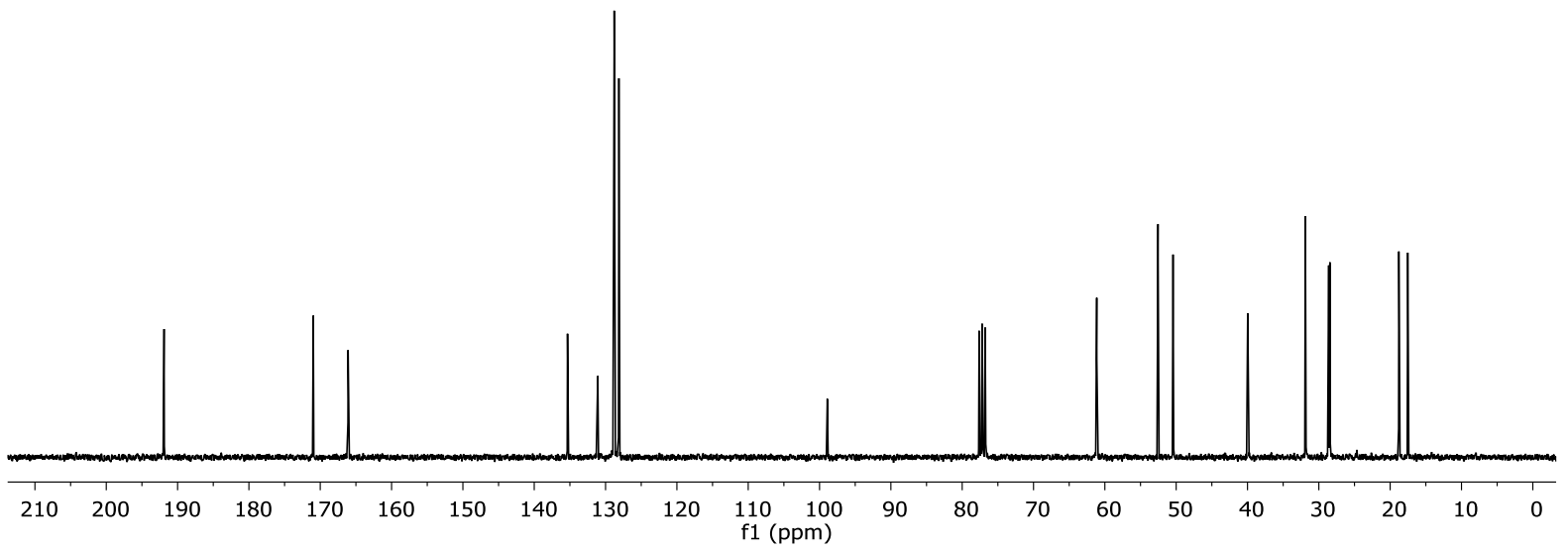

Figure S30: ${ }^{13} \mathrm{C}\left\{{ }^{1} \mathrm{H}\right\}$ NMR spectrum of compound 50 in $\mathrm{CDCl}_{3}(75 \mathrm{MHz})$ 
㟧

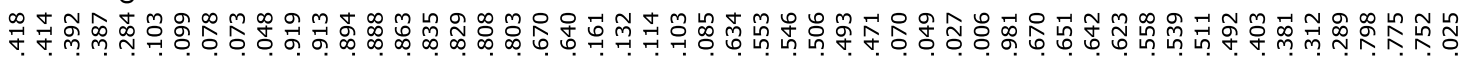
…<smiles>COC(=O)C(CC(C)C)Nc1ccccc1Sc1sc(=O)ccc1Br</smiles>

$5 p$

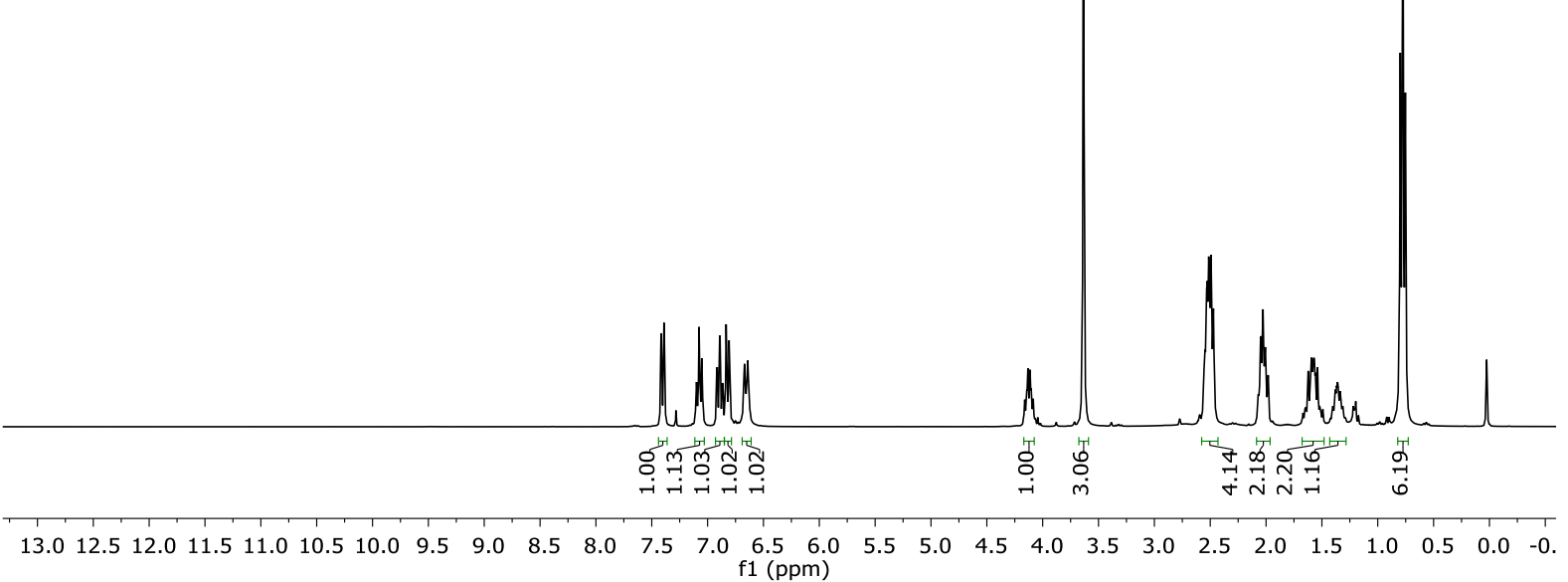

Figure S31: ${ }^{1} \mathrm{H}$ NMR spectrum of compound 5p in $\mathrm{CDCl}_{3}(300 \mathrm{MHz})$<smiles>COC(=O)C(CC(C)C)Nc1ccccc1SC1=C(Br)CCCC1=O</smiles>

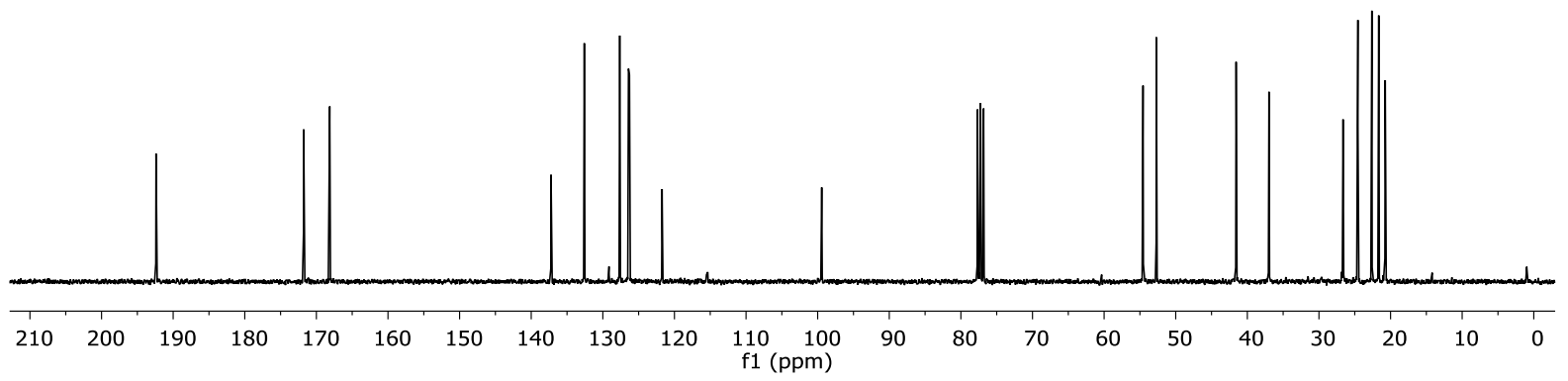

Figure S32: ${ }^{13} \mathrm{C}\left\{{ }^{1} \mathrm{H}\right\}$ NMR spectrum of compound 5p in $\mathrm{CDCl}_{3}(75 \mathrm{MHz})$ 


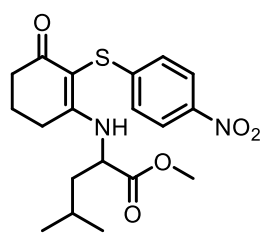

$5 q$

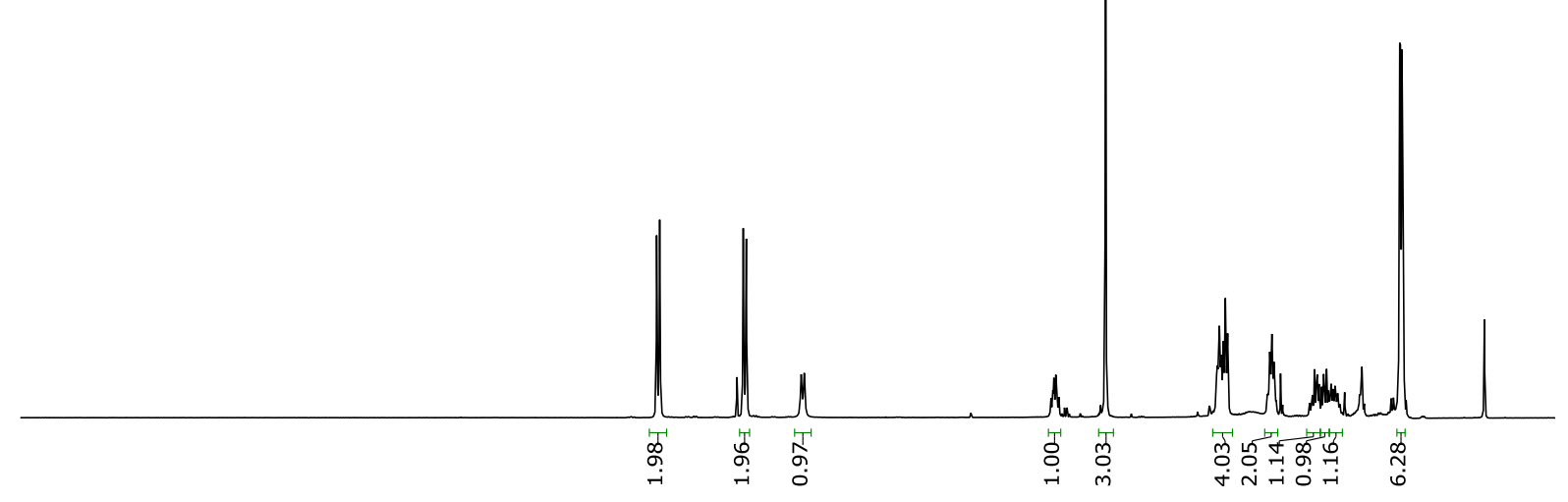

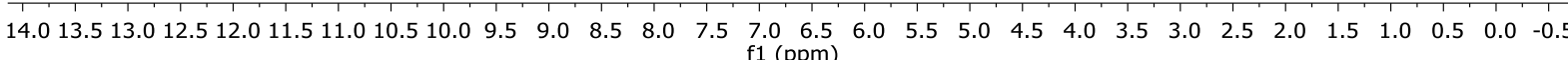

Figure S33: ${ }^{1} \mathrm{H}$ NMR spectrum of compound $\mathbf{5 q}$ in $\mathrm{CDCl}_{3}(300 \mathrm{MHz})$

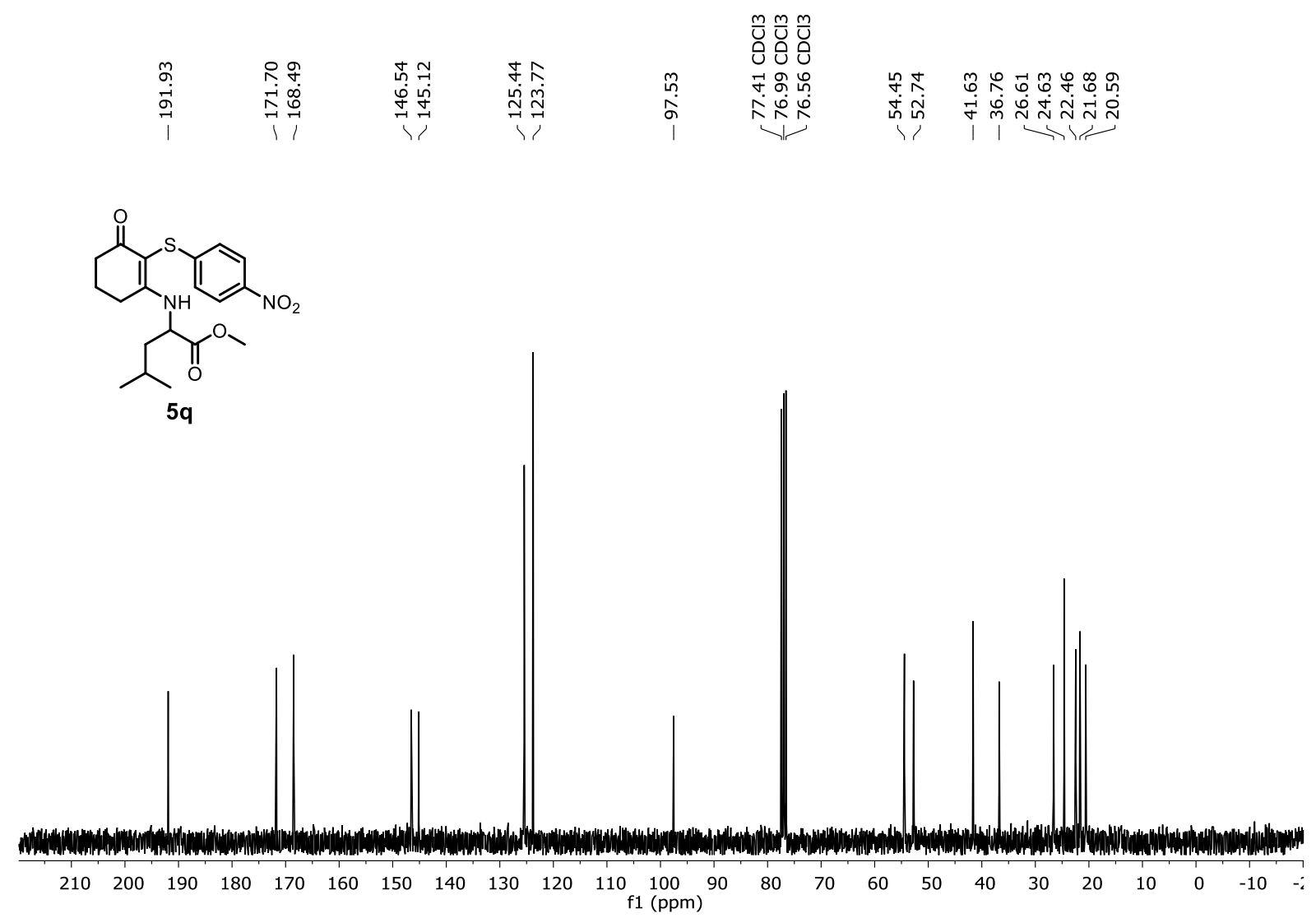

Figure S34: ${ }^{13} \mathrm{C}\left\{{ }^{1} \mathrm{H}\right\}$ NMR spectrum of compound $\mathbf{5 q}$ in $\mathrm{CDCl}_{3}(75 \mathrm{MHz})$ 
<smiles>COC(=O)C(CC(C)Br)N/C(C)=C(/Sc1ccc(Br)cc1)C(=O)c1ccccc1</smiles>

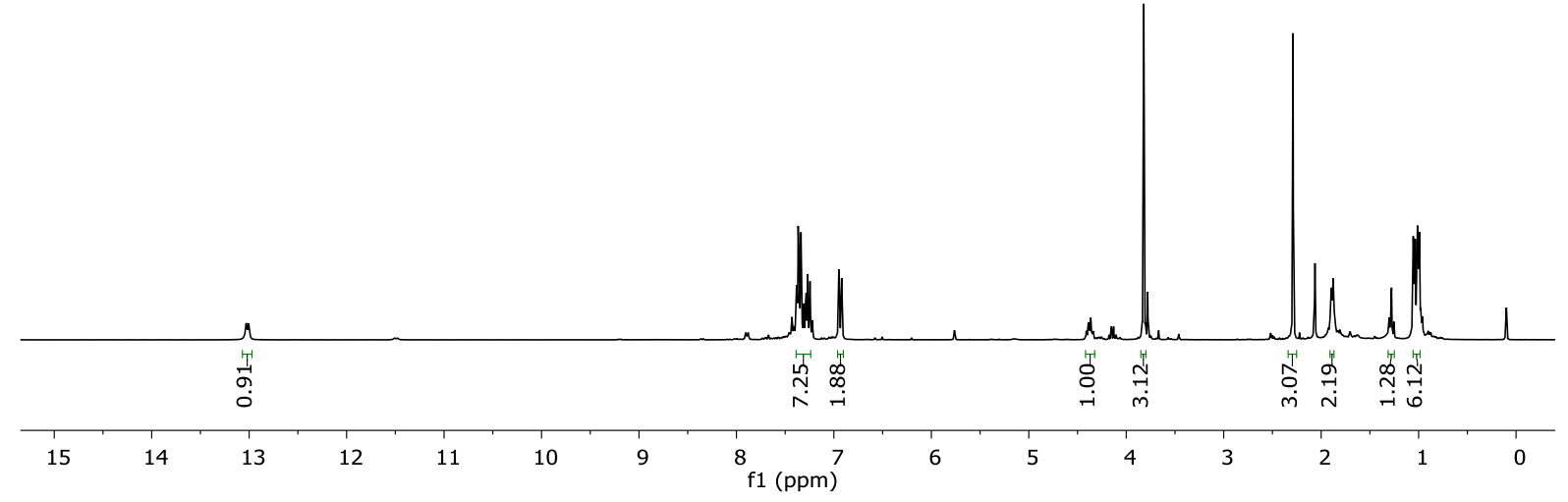

Figure S35: ${ }^{1} \mathrm{H}$ NMR spectrum of compound $5 \mathbf{r}$ in $\mathrm{CDCl}_{3}(300 \mathrm{MHz})$

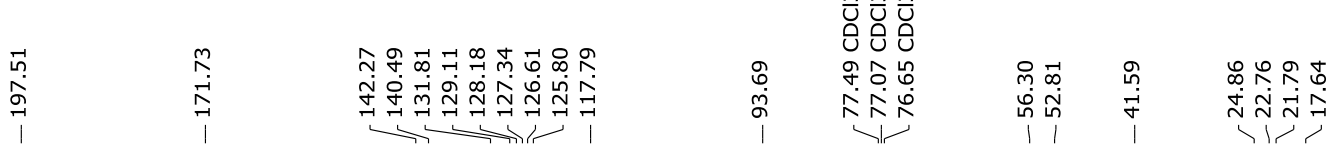<smiles>COC(=O)C(CC(C)Br)N/C(C)=C(/Sc1ccc(Br)cc1)C(=O)c1ccccc1</smiles>

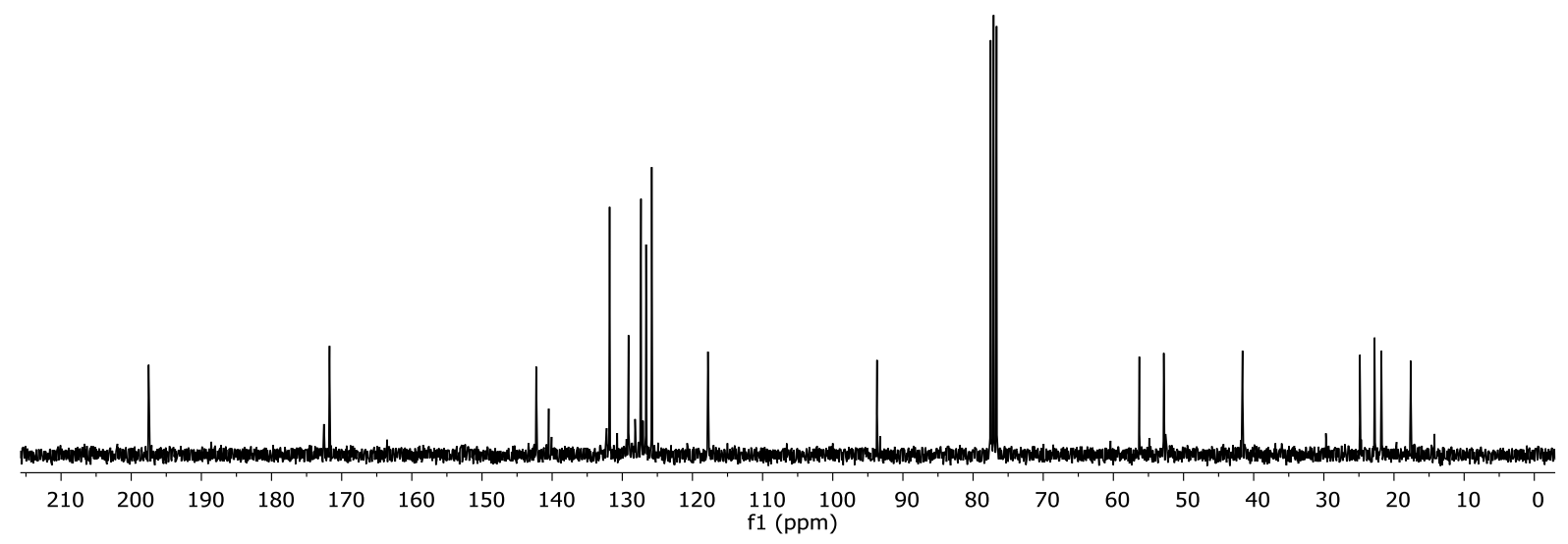

Figure S36: ${ }^{13} \mathrm{C}\left\{{ }^{1} \mathrm{H}\right\}$ NMR spectrum of compound $\mathbf{5 r}$ in $\mathrm{CDCl}_{3}(75 \mathrm{MHz})$ 
<smiles>COC(=O)C(Cc1c[nH]c2ccccc12)Nc1ccccc1Sc1ccccc1Br</smiles>

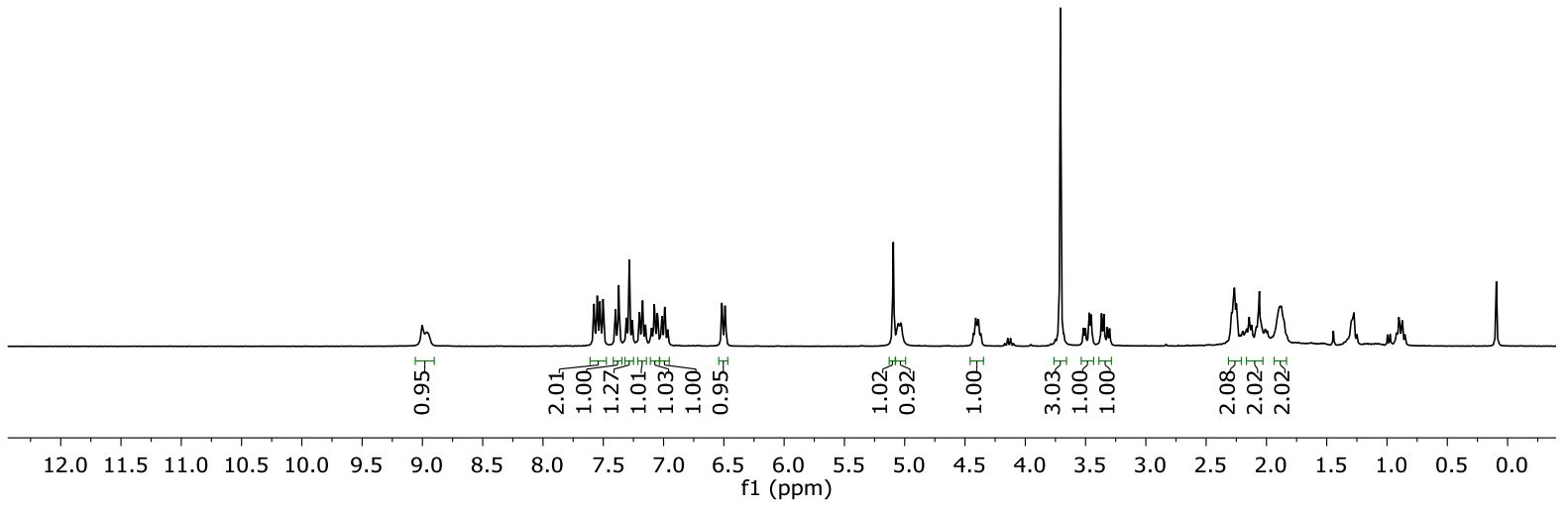

Figure S37: ${ }^{1} \mathrm{H}$ NMR spectrum of compound $\mathbf{5 s}$ in $\mathrm{CDCl}_{3}(300 \mathrm{MHz})$<smiles></smiles>

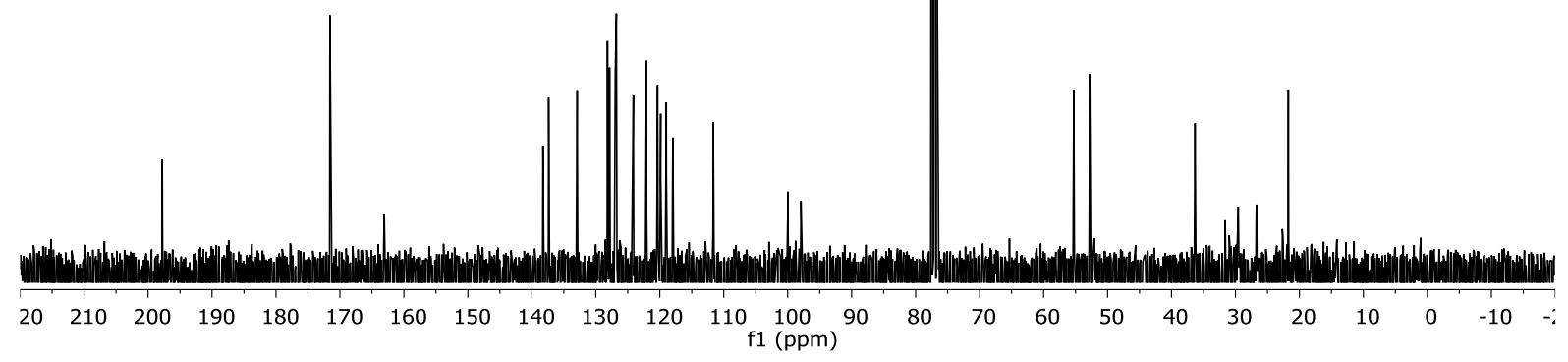

Figure S38: ${ }^{13} \mathrm{C}\left\{{ }^{1} \mathrm{H}\right\}$ NMR spectrum of compound $\mathbf{5 s}$ in $\mathrm{CDCl}_{3}(75 \mathrm{MHz})$ 
<smiles>COC(=O)/C(NC(Cc1c[nH]c2ccccc12)C(C)=O)=C(/Sc1ccc(C)cc1)C(C)=O</smiles>

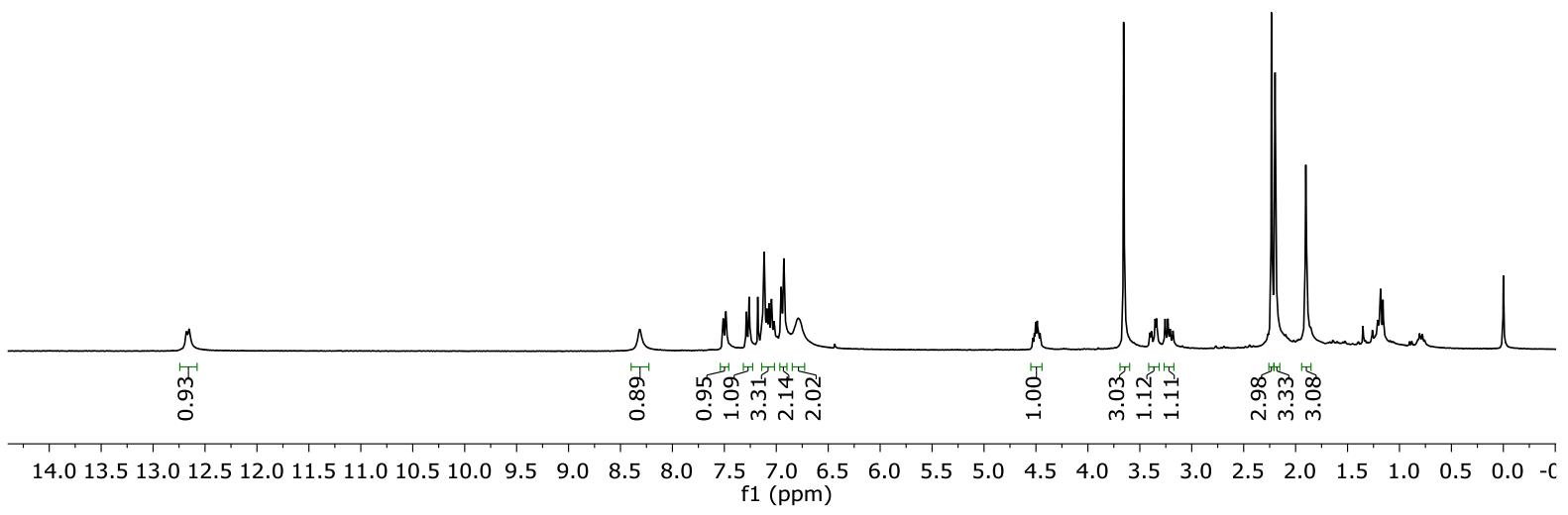

Figure S39: ${ }^{1} \mathrm{H}$ NMR spectrum of compound $\mathbf{5 t}$ in $\mathrm{CDCl}_{3}(300 \mathrm{MHz})$<smiles>COC(=O)C(Cc1c[nH]c2ccccc12)NC(C)=C(Sc1ccc(C)cc1)C(C)=O</smiles>

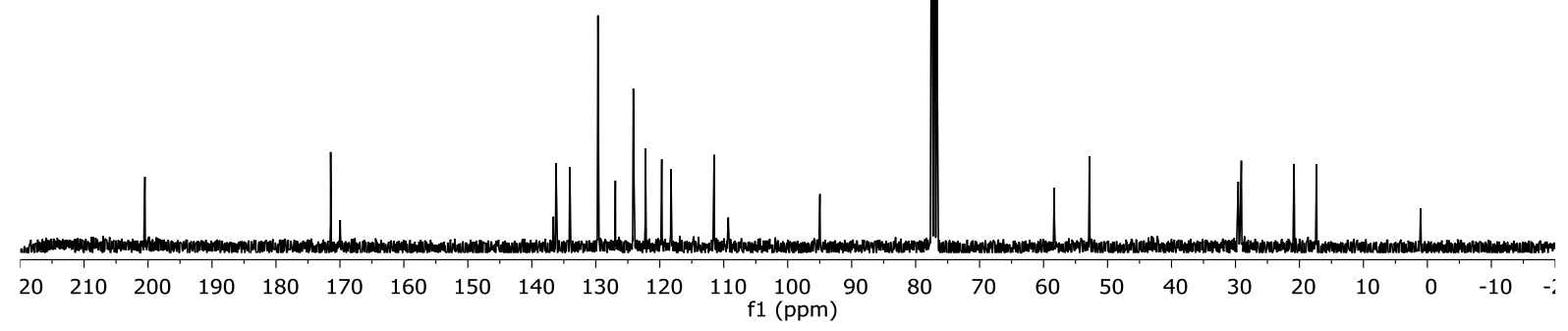

Figure S40: ${ }^{13} \mathrm{C}\left\{{ }^{1} \mathrm{H}\right\}$ NMR spectrum of compound $\mathbf{5 t}$ in $\mathrm{CDCl}_{3}(75 \mathrm{MHz})$ 
$\stackrel{\mathrm{m}}{\mathrm{u}}$

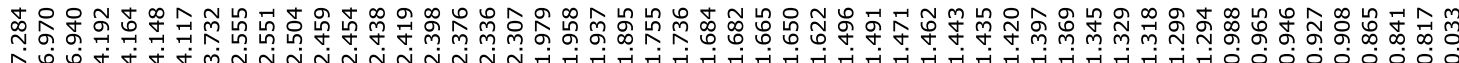<smiles>CCCCSC1=C(NC(CC(C)C)C(=O)OC)CCCC1=O</smiles>

$5 u$

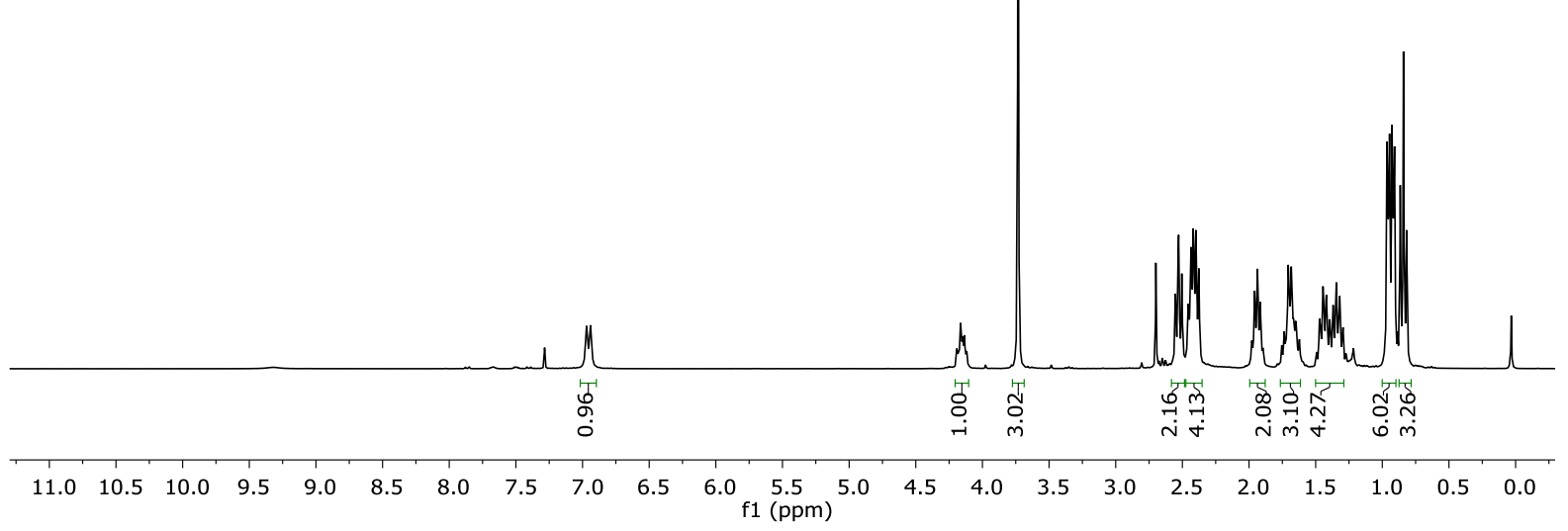

Figure S41: ${ }^{1} \mathrm{H}$ NMR spectrum of compound $\mathbf{5 u}$ in $\mathrm{CDCl}_{3}(300 \mathrm{MHz})$

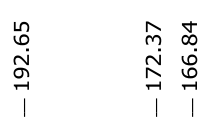<smiles>CCCCSC1=C(NC(CC(C)C)C(=O)OC)CCCC1=O</smiles>

$5 u$

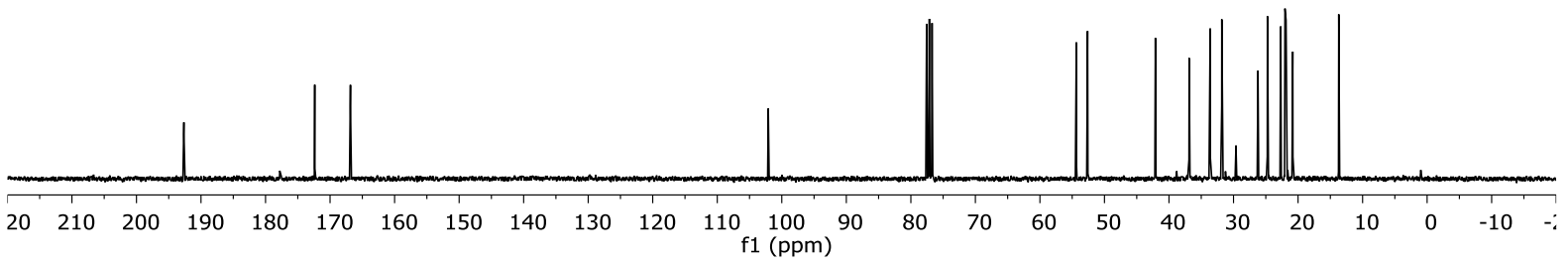

Figure S42: ${ }^{13} \mathrm{C}\left\{{ }^{1} \mathrm{H}\right\}$ NMR spectrum of compound $\mathbf{5 u}$ in $\mathrm{CDCl}_{3}(75 \mathrm{MHz})$ 
రัญ

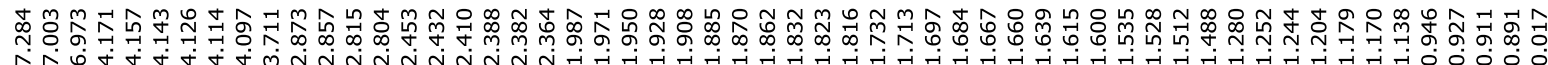

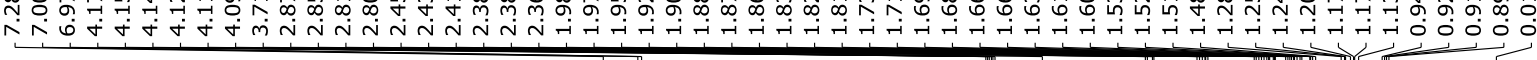<smiles>COC(=O)C(CC(C)C)NC1=C(SC2CCCC2)C(=O)CC1</smiles>

$5 v$

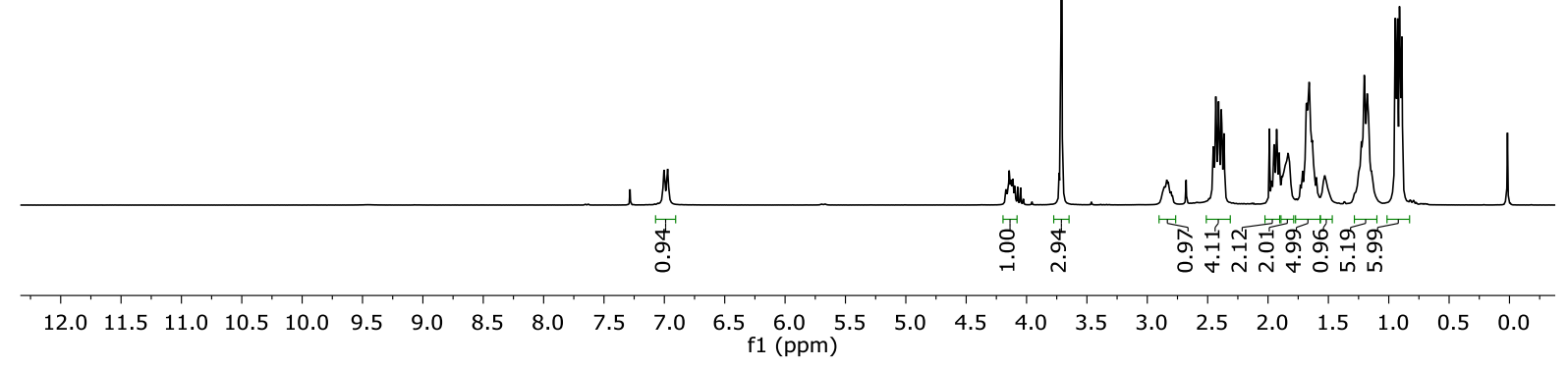

Figure S43: ${ }^{1} \mathrm{H}$ NMR spectrum of compound 5v in $\mathrm{CDCl}_{3}(300 \mathrm{MHz})$
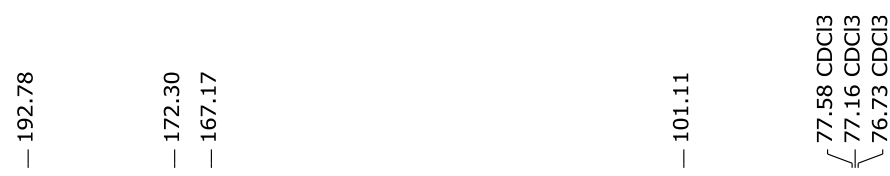

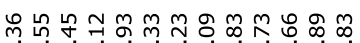

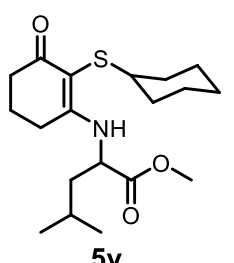

$5 v$

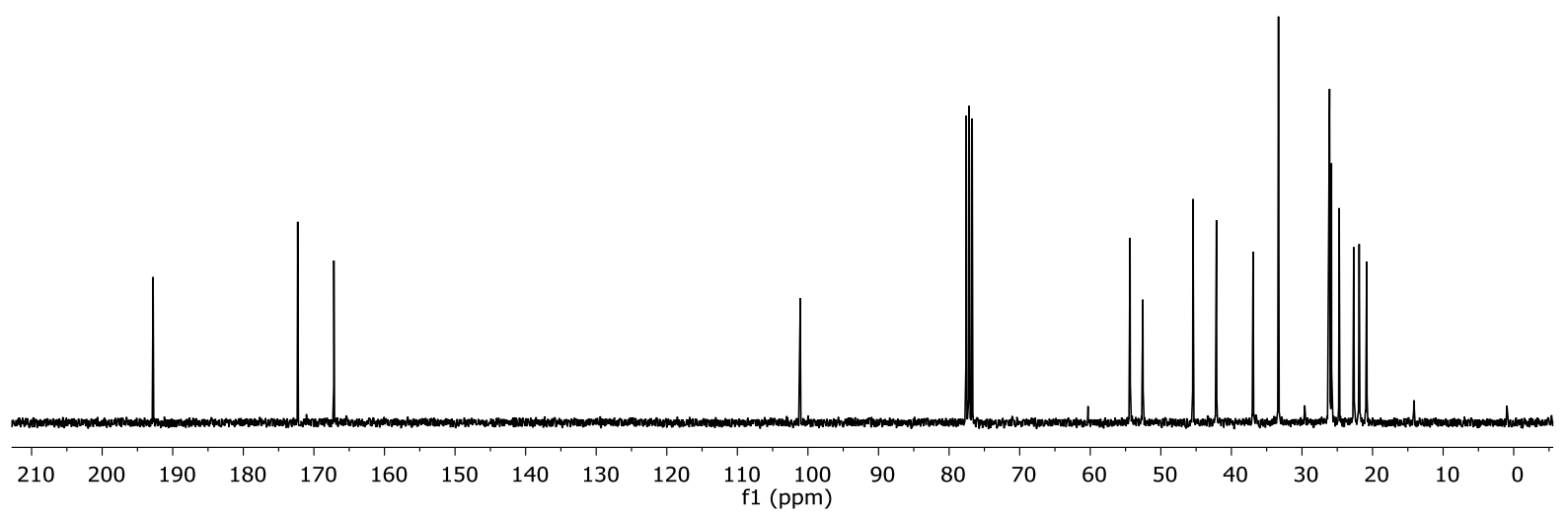

Figure S44: ${ }^{13} \mathrm{C}\left\{{ }^{1} \mathrm{H}\right\}$ NMR spectrum of compound $\mathbf{5 v}$ in $\mathrm{CDCl}_{3}(75 \mathrm{MHz})$ 
<smiles>COC(=O)C(Cc1ccccc1)N/C(C(C)=O)=C(/Sc1nc2ccccc2s1)C(=O)O</smiles>

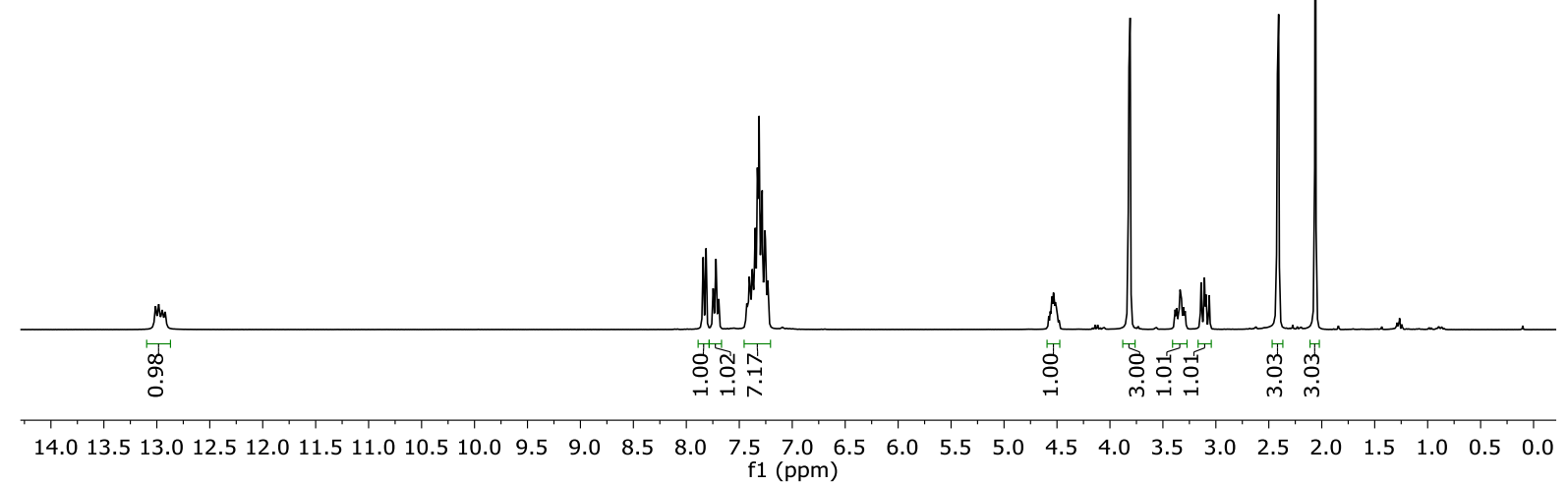

Figure S45: ${ }^{1} \mathrm{H}$ NMR spectrum of compound $\mathbf{5 w}$ in $\mathrm{CDCl}_{3}(300 \mathrm{MHz})$

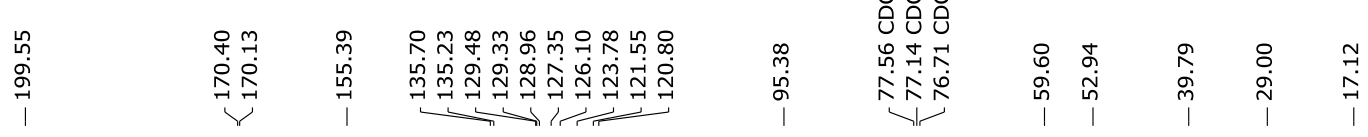<smiles>COC(=O)/C(Sc1nc2ccccc2s1)=C(\NC(Cc1ccccc1)C(=O)OC)C(C)=O</smiles>

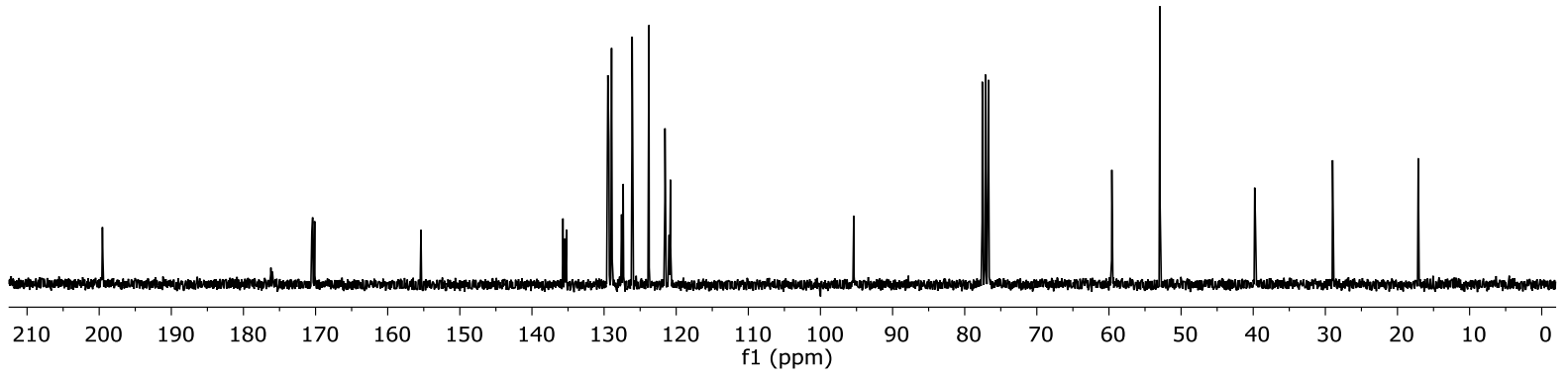

Figure S46: ${ }^{13} \mathrm{C}\left\{{ }^{1} \mathrm{H}\right\}$ NMR spectrum of compound $\mathbf{5 w}$ in $\mathrm{CDCl}_{3}(75 \mathrm{MHz})$ 
<smiles>COC(=O)C(Nc1nc2ccccc2s1)N1CC(C)(C)CC(=O)C1=O</smiles>

$5 x$

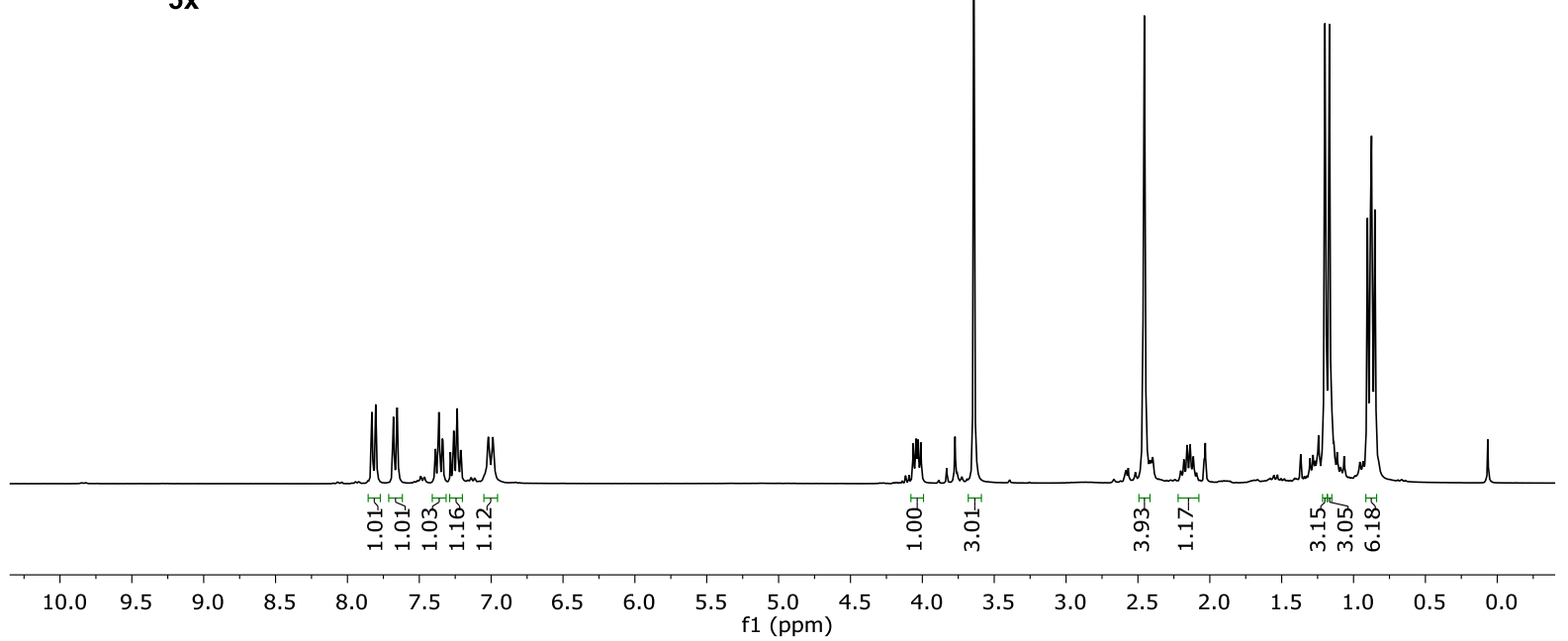

Figure S47: ${ }^{1} \mathrm{H}$ NMR spectrum of compound $\mathbf{5 x}$ in $\mathrm{CDCl}_{3}(300 \mathrm{MHz})$

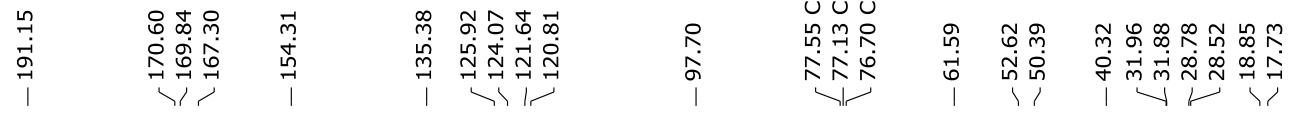<smiles>COC(=O)C(Nc1nc2ccccc2s1)C(C(=O)OC)N1CC(C)(C)CC(=O)C1=O</smiles>

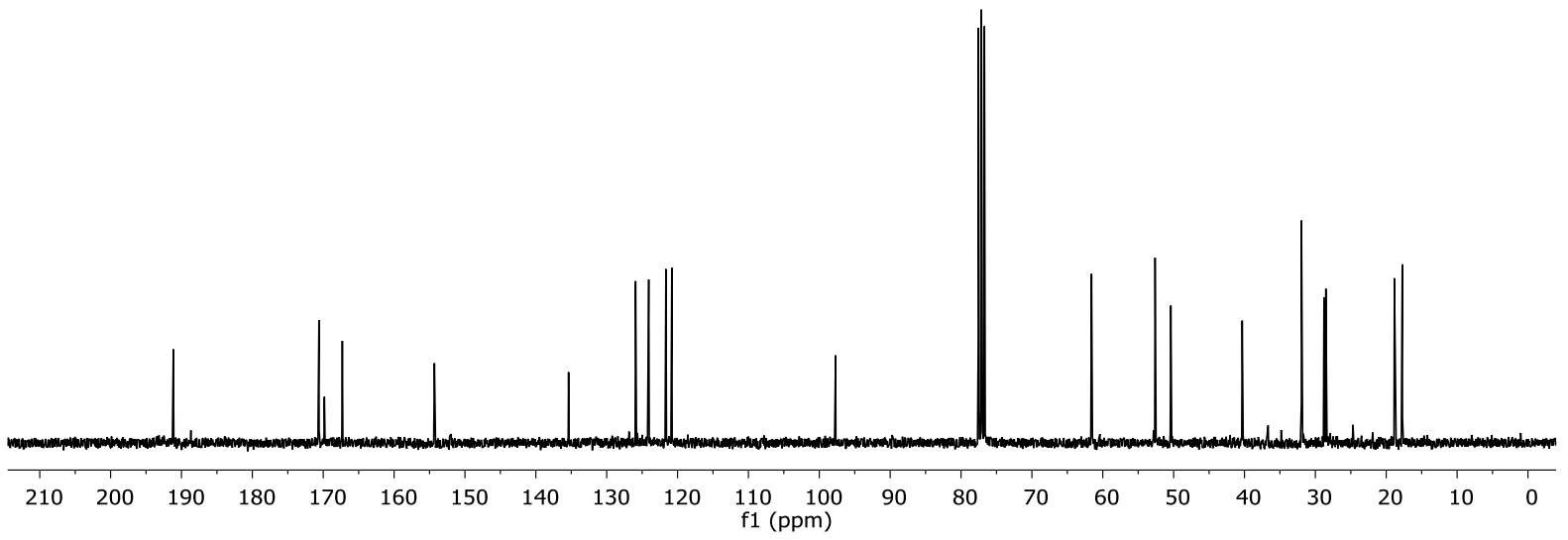

Figure S48: ${ }^{13} \mathrm{C}\left\{{ }^{1} \mathrm{H}\right\}$ NMR spectrum of compound $\mathbf{5 x}$ in $\mathrm{CDCl}_{3}(75 \mathrm{MHz})$ 
<smiles></smiles>

$5 y$

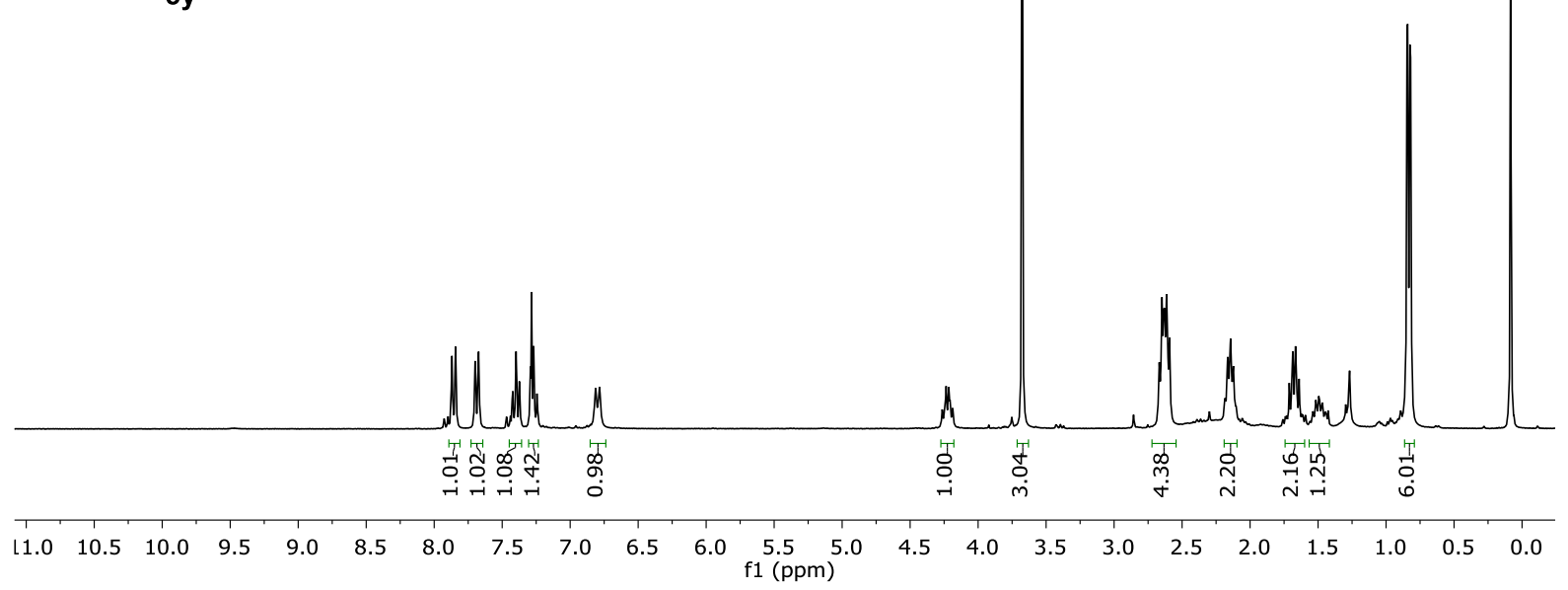

Figure S49: ${ }^{1} \mathrm{H}$ NMR spectrum of compound $5 \mathbf{y}$ in $\mathrm{CDCl}_{3}(300 \mathrm{MHz})$

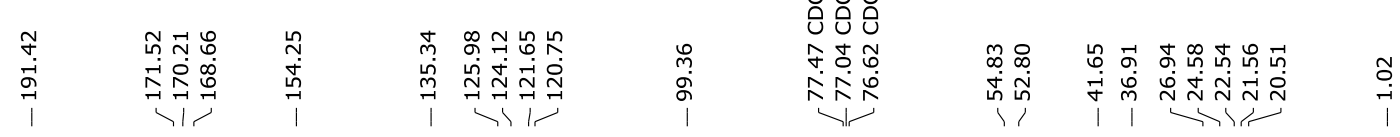<smiles>COC(=O)C(CC(C)C)Nc1sc2nc3cccc-3sc2c1C</smiles>

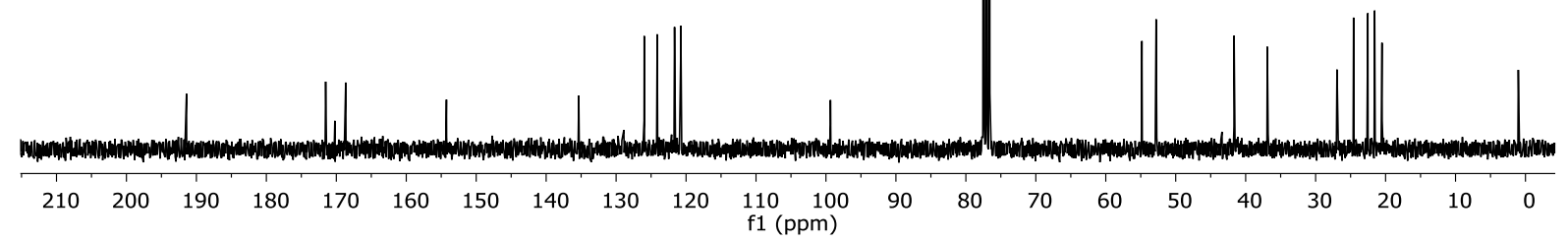

Figure S50: ${ }^{13} \mathrm{C}\left\{{ }^{1} \mathrm{H}\right\}$ NMR spectrum of compound $\mathbf{5 y}$ in $\mathrm{CDCl}_{3}(75 \mathrm{MHz})$ 
రై

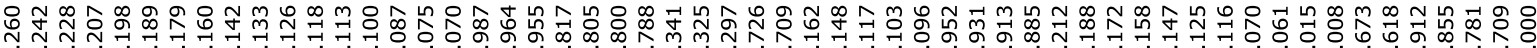

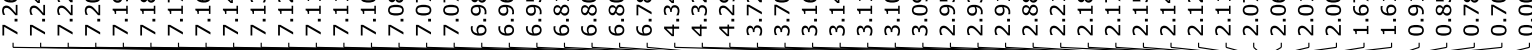<smiles></smiles>

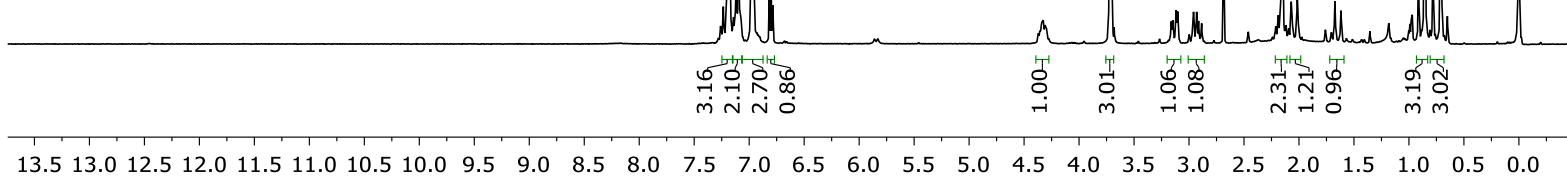

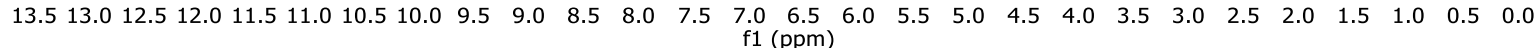

Figure S51: ${ }^{1} \mathrm{H}$ NMR spectrum of compound $\mathbf{5 z}$ in $\mathrm{CDCl}_{3}(300 \mathrm{MHz})$

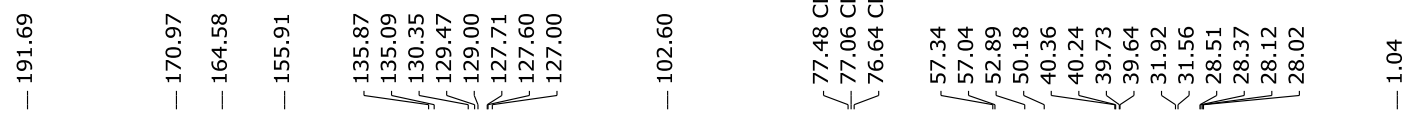<smiles></smiles>

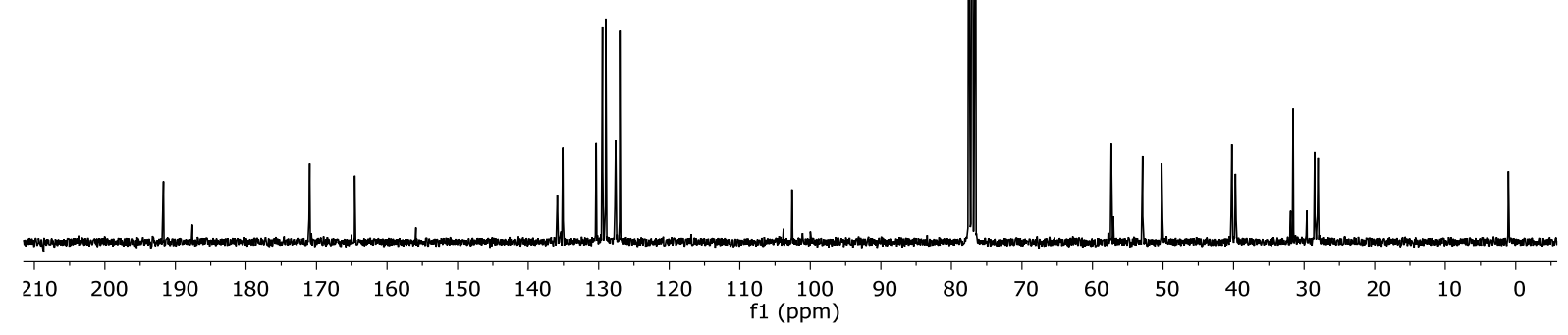

Figure S52: ${ }^{13} \mathrm{C}\left\{{ }^{1} \mathrm{H}\right\}$ NMR spectrum of compound $\mathbf{5 z}$ in $\mathrm{CDCl}_{3}(75 \mathrm{MHz})$ 
网

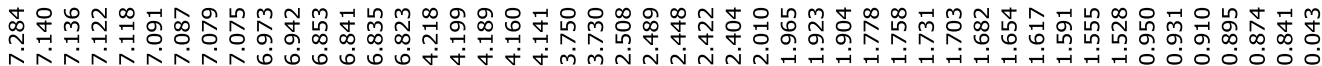<smiles>COC(=O)C(CC(C)Br)Nc1cccc2sc3c(c1)CCCC23Br</smiles>

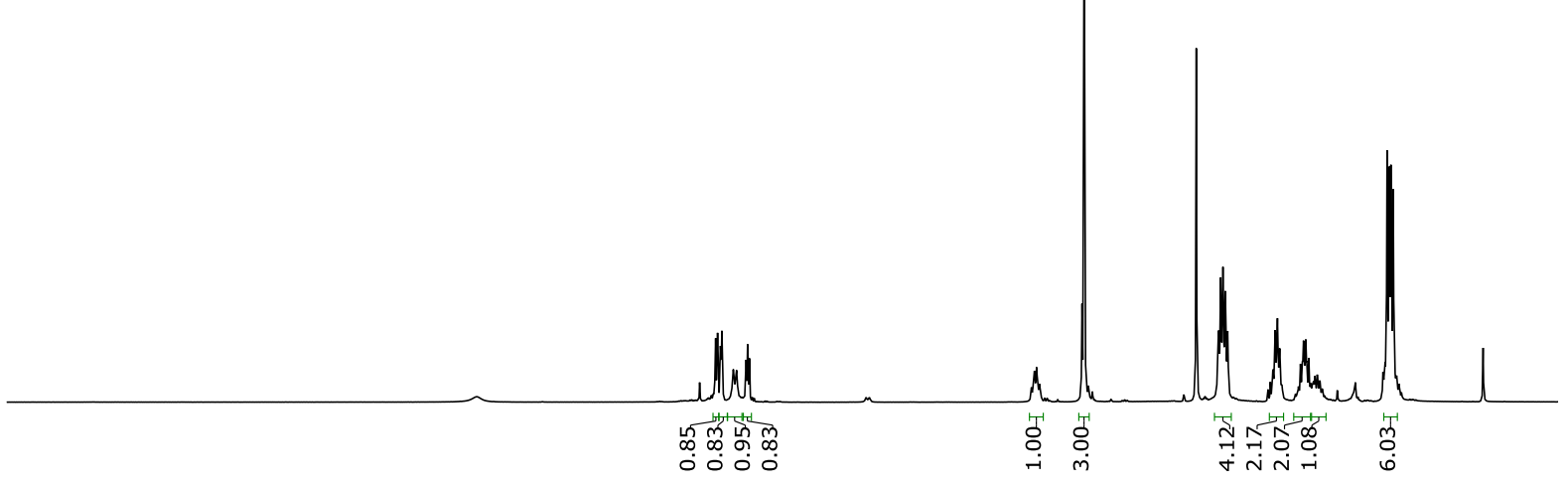

$\begin{array}{lllllllllllllllllllllllllllllllll}13.5 & 13.0 & 12.5 & 12.0 & 11.5 & 11.0 & 10.5 & 10.0 & 9.5 & 9.0 & 8.5 & 8.0 & 7.5 & 7.0 & 6.5 & 6.0 & 5.5 & 5.0 & 4.5 & 4.0 & 3.5 & 3.0 & 2.5 & 2.0 & 1.5 & 1.0 & 0.5 & 0.0 & -0.1\end{array}$

Figure S53: ${ }^{1} \mathrm{H}$ NMR spectrum of compound 5a' in $\mathrm{CDCl}_{3}(300 \mathrm{MHz})$

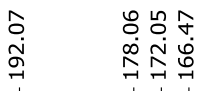
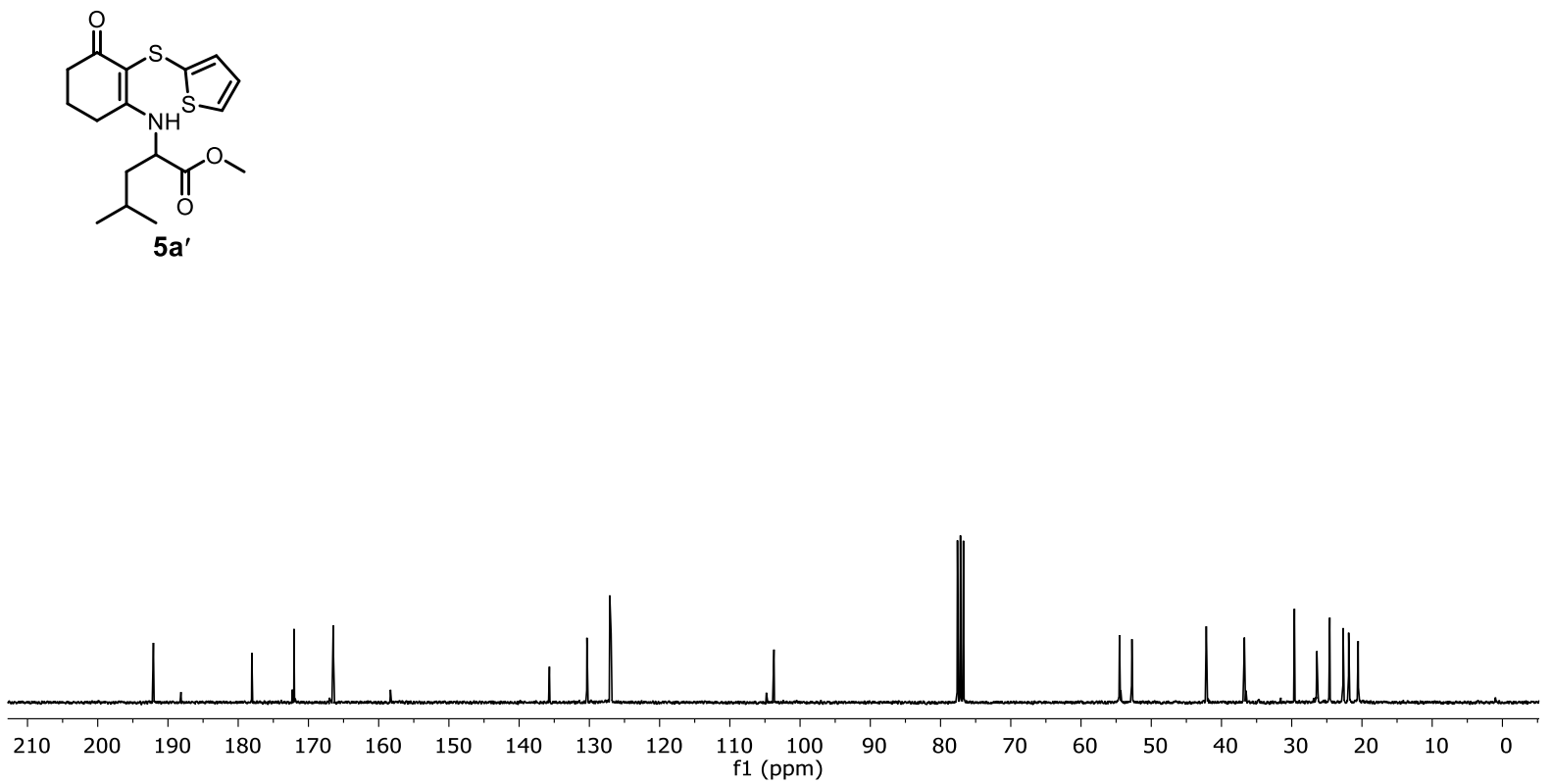

Figure S54: ${ }^{13} \mathrm{C}\left\{{ }^{1} \mathrm{H}\right\}$ NMR spectrum of compound $\mathbf{5 a}$ ' in $\mathrm{CDCl}_{3}(75 \mathrm{MHz})$ 


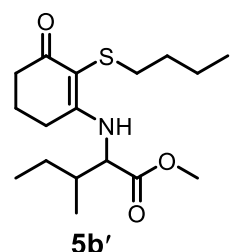

$5 b^{\prime}$

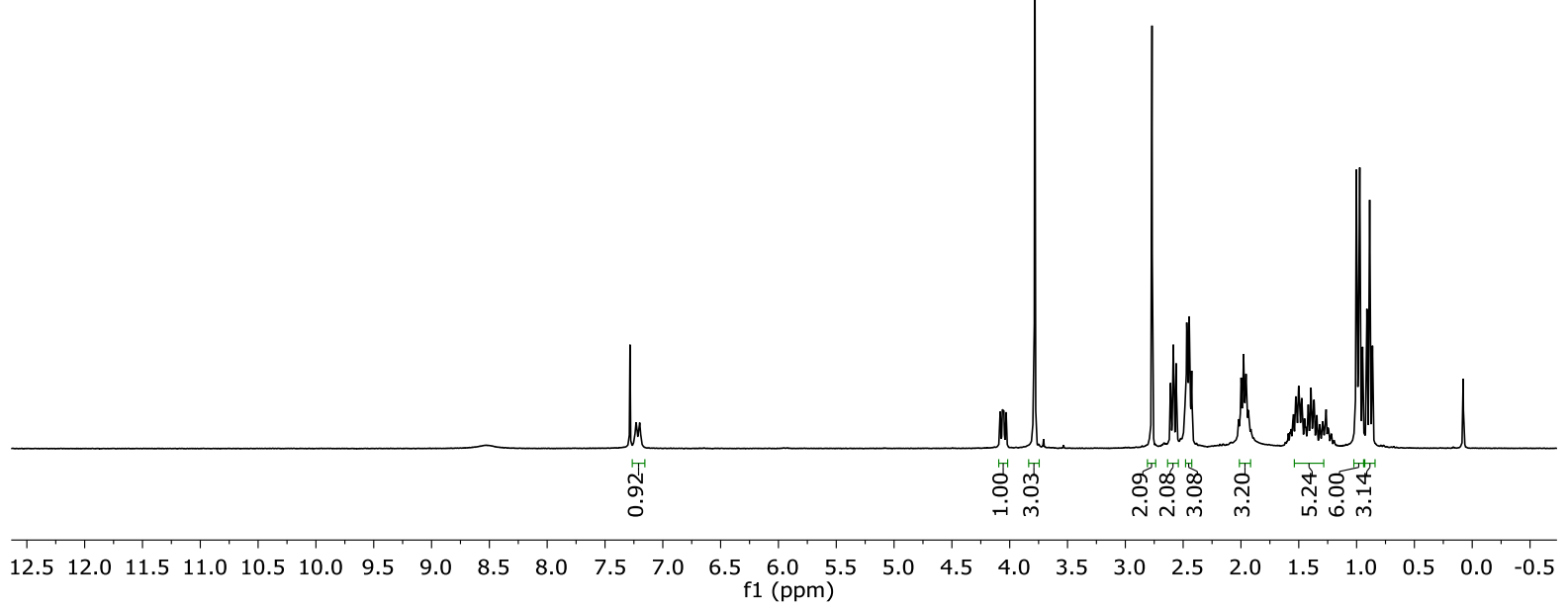

Figure S55: ${ }^{1} \mathrm{H}$ NMR spectrum of compound $\mathbf{5 b} \mathbf{b}^{\prime}$ in $\mathrm{CDCl}_{3}(300 \mathrm{MHz})$

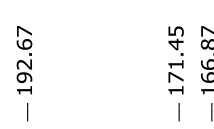
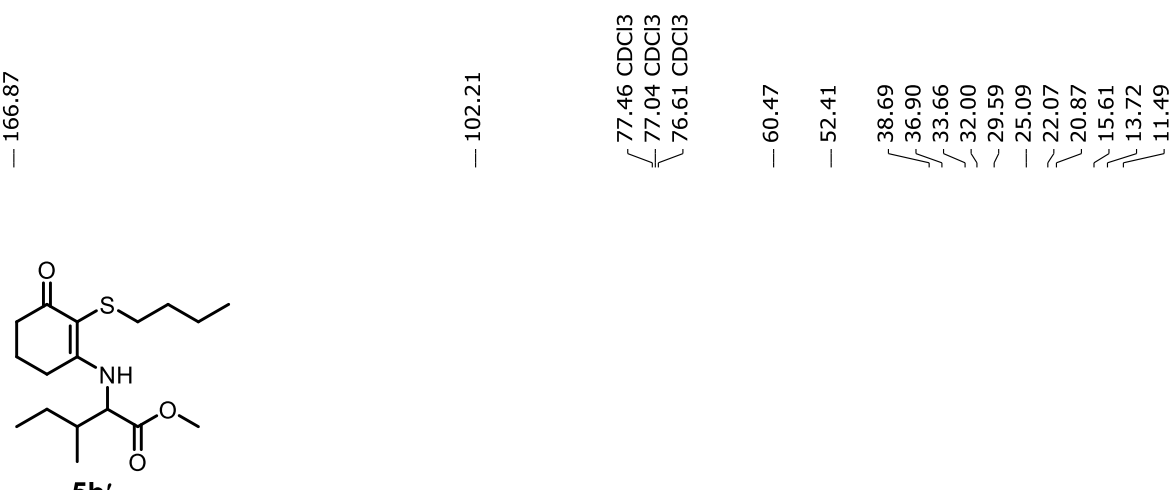

$5 b^{\prime}$

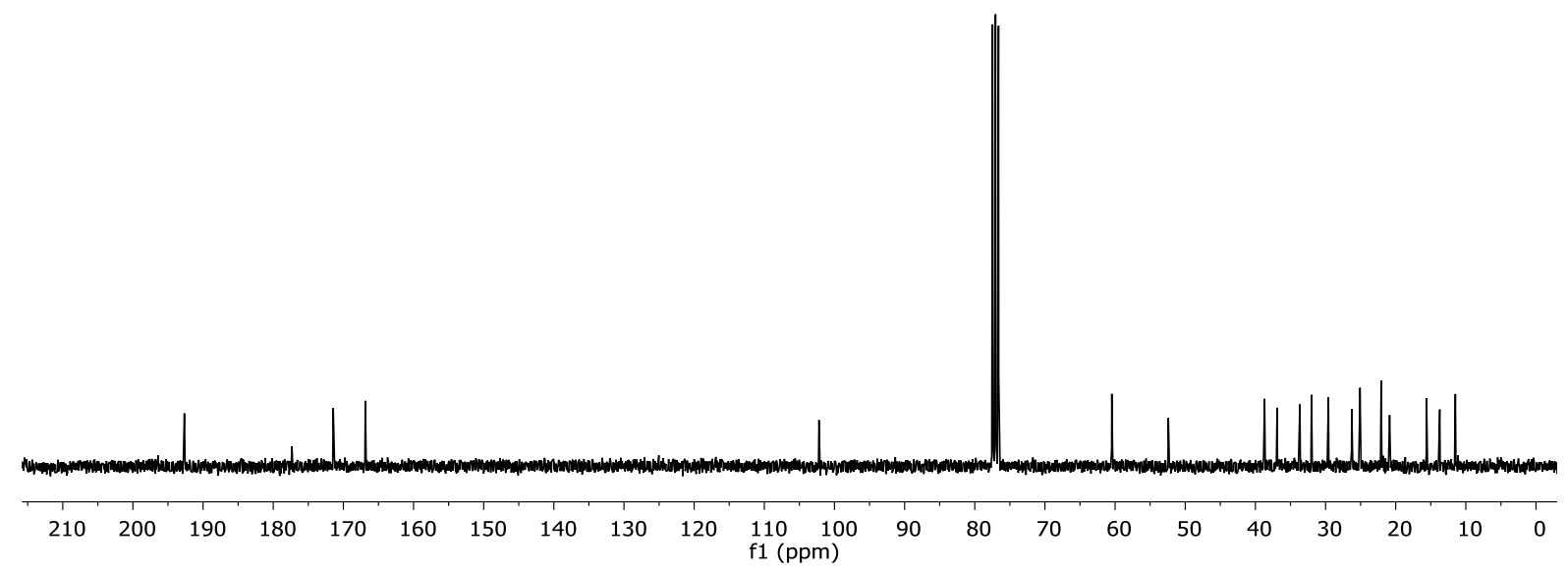

Figure S56: ${ }^{13} \mathrm{C}\left\{{ }^{1} \mathrm{H}\right\}$ NMR spectrum of compound $\mathbf{5} \mathbf{b}^{\prime}$ in $\mathrm{CDCl}_{3}(75 \mathrm{MHz})$ 


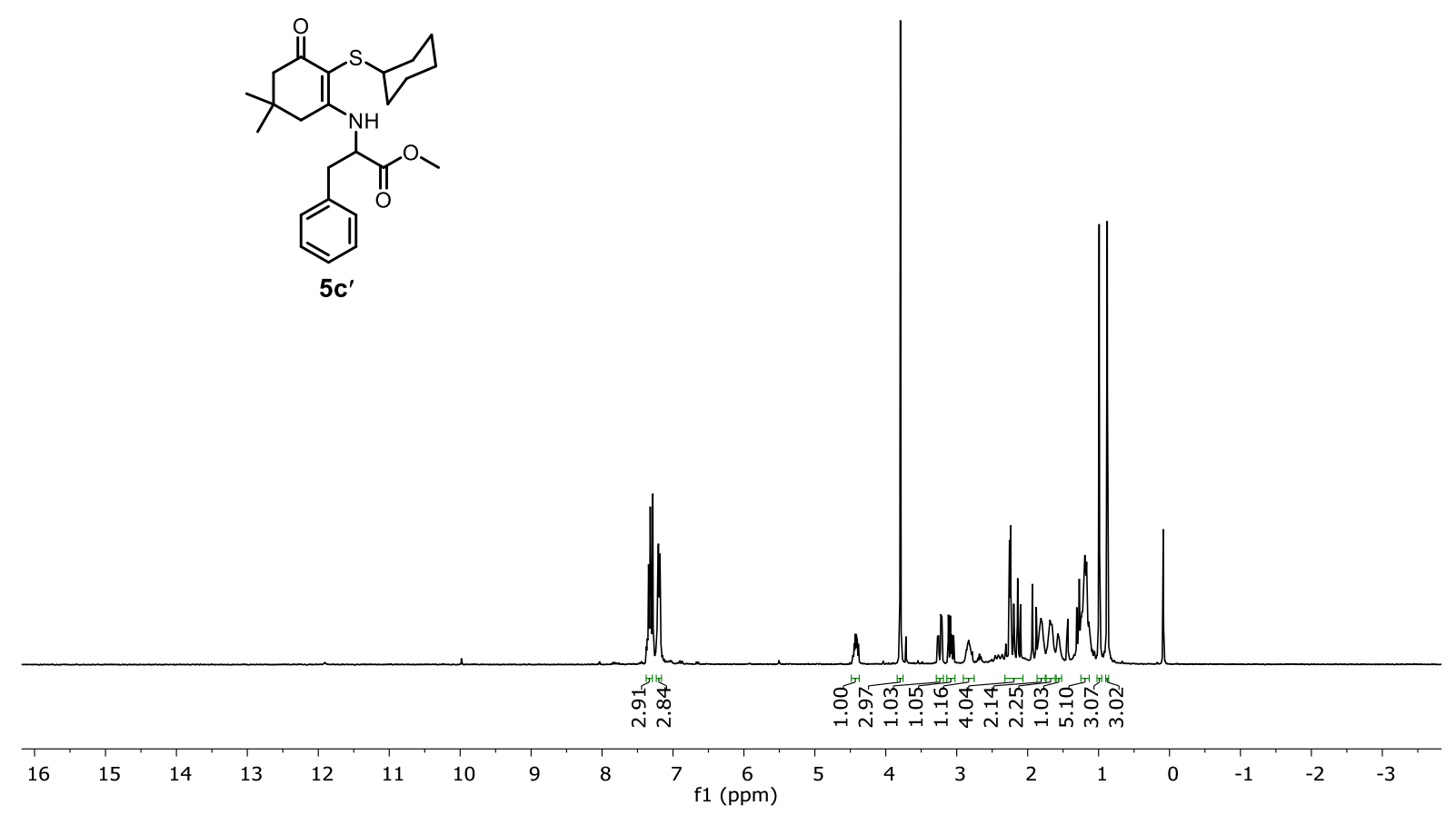

Figure S57: ${ }^{1} \mathrm{H}$ NMR spectrum of compound $\mathbf{5} \mathbf{c}^{\prime}$ in $\mathrm{CDCl}_{3}(300 \mathrm{MHz})$
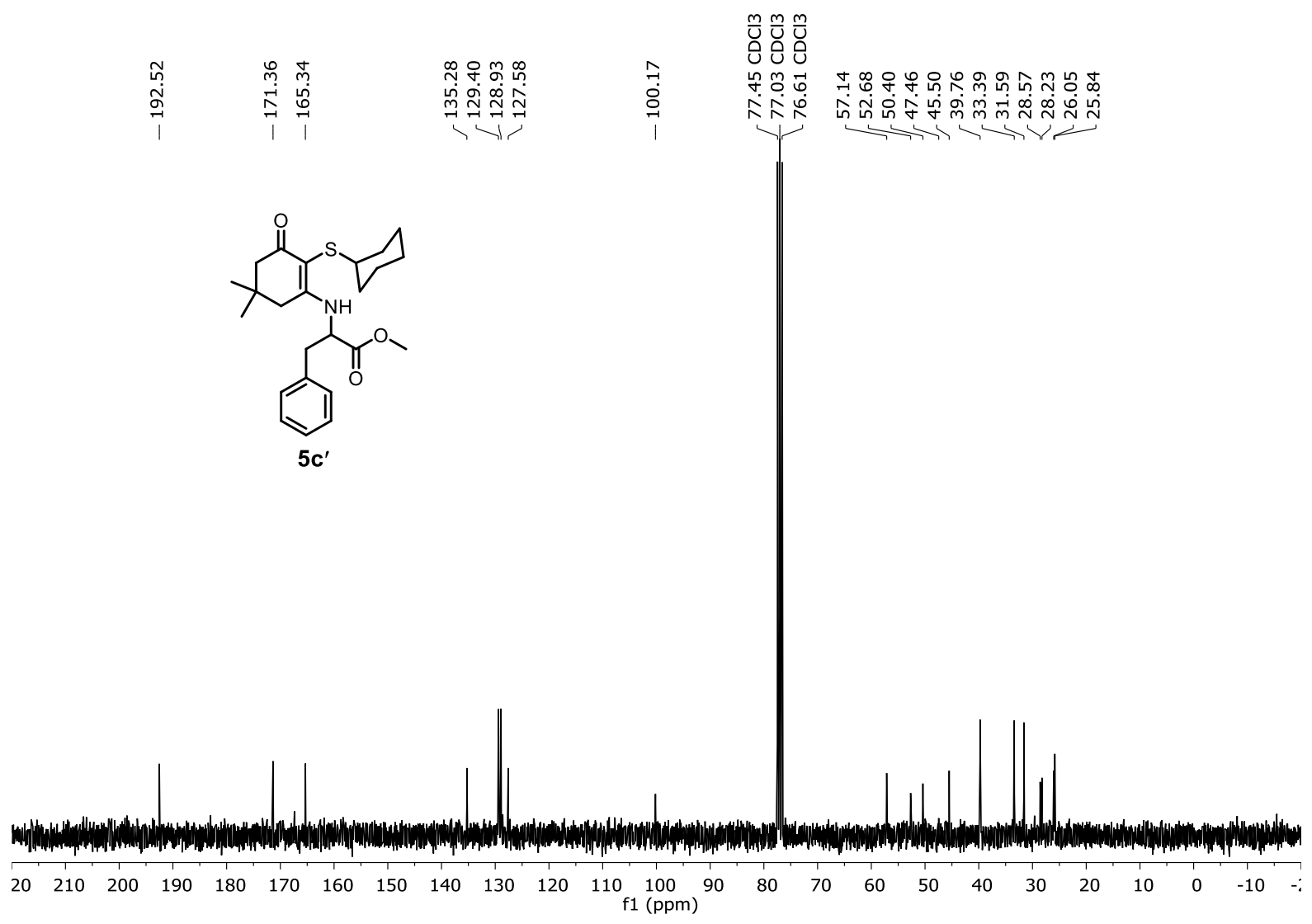

Figure S58: ${ }^{13} \mathrm{C}\left\{{ }^{1} \mathrm{H}\right\}$ NMR spectrum of compound $\mathbf{5} \mathbf{c}^{\prime}$ in $\mathrm{CDCl}_{3}(75 \mathrm{MHz})$ 


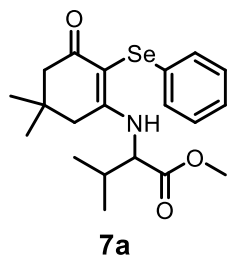

$7 \mathrm{a}$

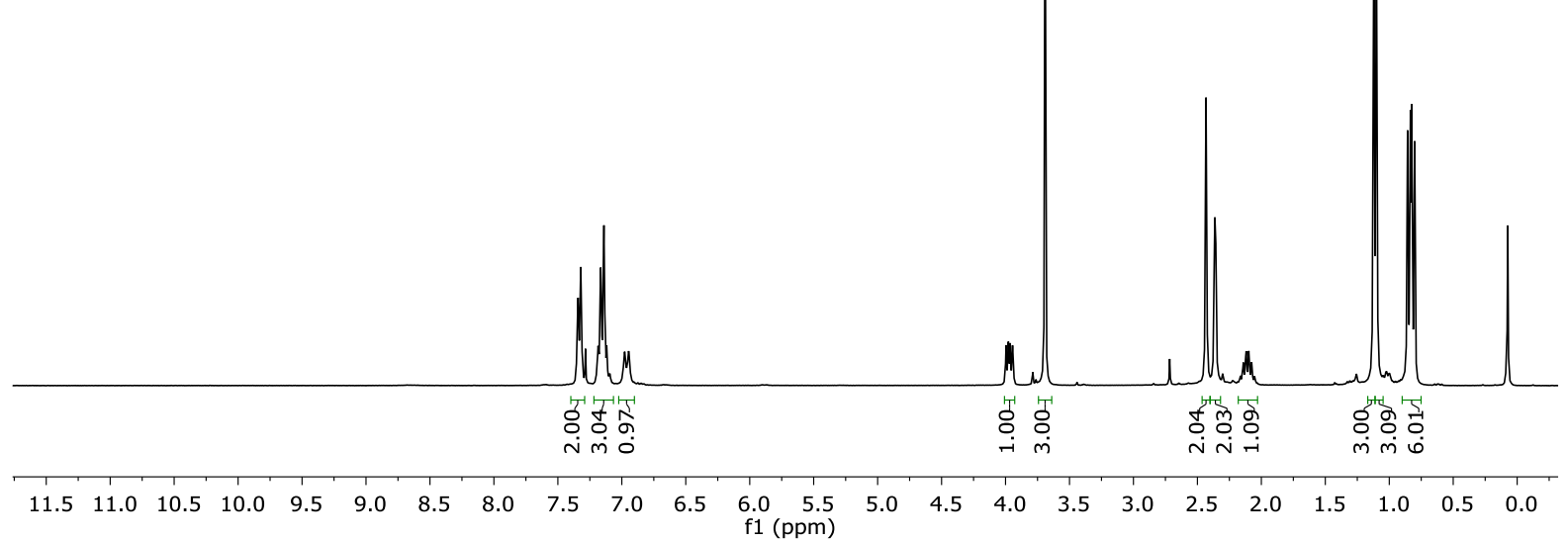

Figure S59: ${ }^{1} \mathrm{H}$ NMR spectrum of compound 7a in $\mathrm{CDCl}_{3}(300 \mathrm{MHz})$

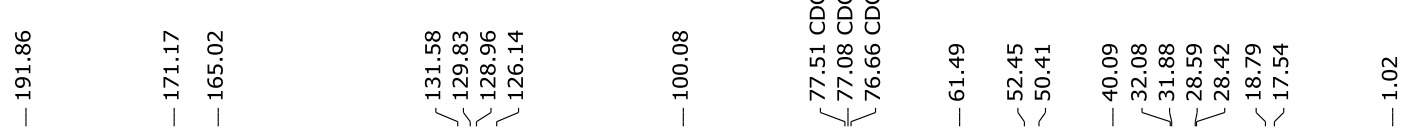<smiles>COC(=O)C(NC1=CC(=O)CC(C)(C)C1)C(C)C</smiles>

$7 a$

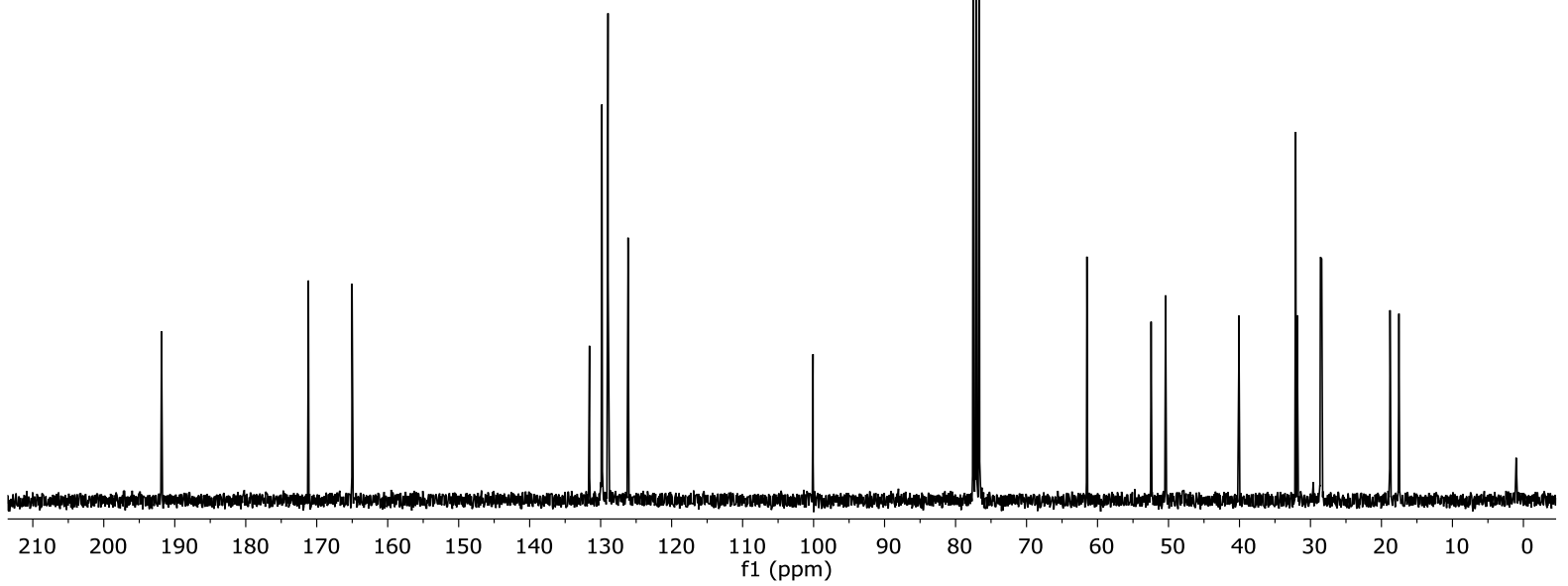

Figure S60: ${ }^{13} \mathrm{C}\left\{{ }^{1} \mathrm{H}\right\}$ NMR spectrum of compound 7a in $\mathrm{CDCl}_{3}(75 \mathrm{MHz})$ 
<smiles>CCC(C)C(Nc1ccccc1)C(=O)OC</smiles>

$7 b$

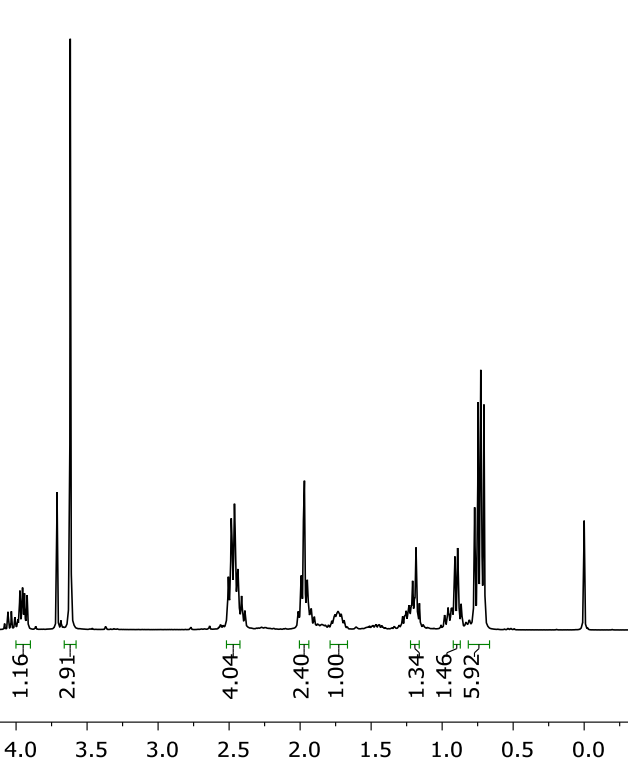

Figure S61: ${ }^{1} \mathrm{H}$ NMR spectrum of compound $7 \mathbf{b}$ in $\mathrm{CDCl}_{3}(300 \mathrm{MHz})$

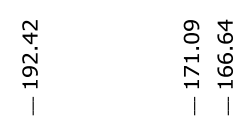<smiles>CCC(C)C(Nc1ccccc1)C1=C(C(=O)OC)C(=O)CCC1</smiles>

$7 b$

\section{$m$ g.}

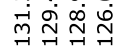

울.
국
I

$m$
$\bar{U}$
$\mathrm{U}$
$\mathrm{u}$
$\mathrm{u}$

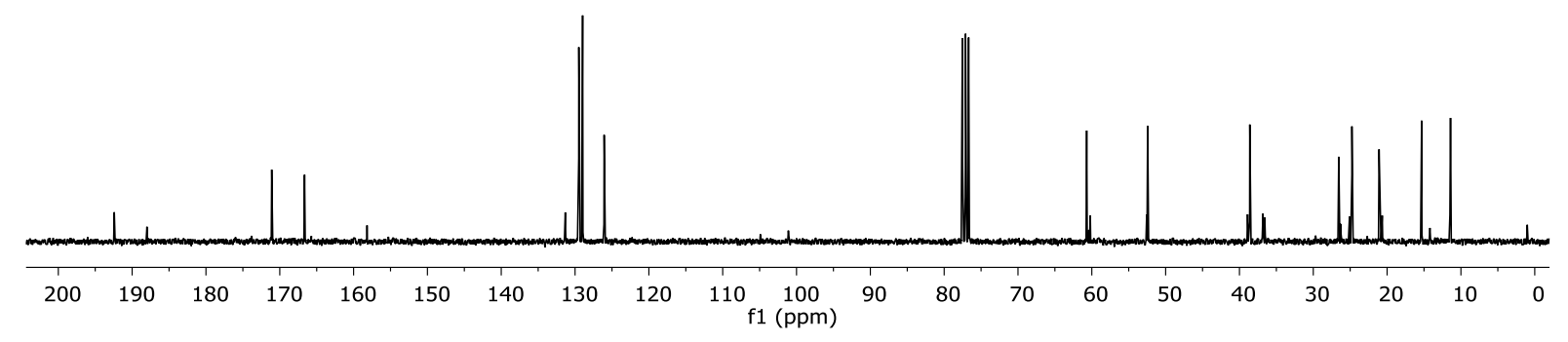

Figure S62: ${ }^{13} \mathrm{C}\left\{{ }^{1} \mathrm{H}\right\}$ NMR spectrum of compound $7 \mathbf{b}$ in $\mathrm{CDCl}_{3}(75 \mathrm{MHz})$ 


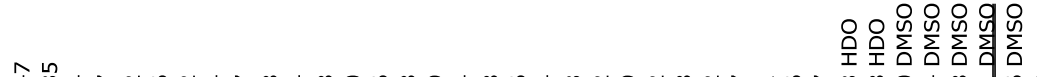

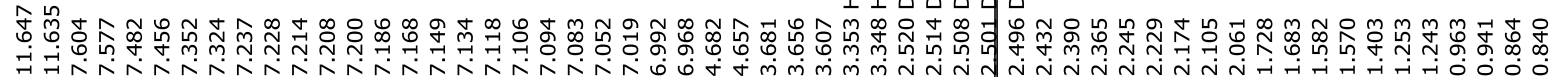<smiles>COC(=O)C(Cc1c[nH]c2ccccc12)NC1=C([Se]c2ccccc2)C(=O)CCC1</smiles>

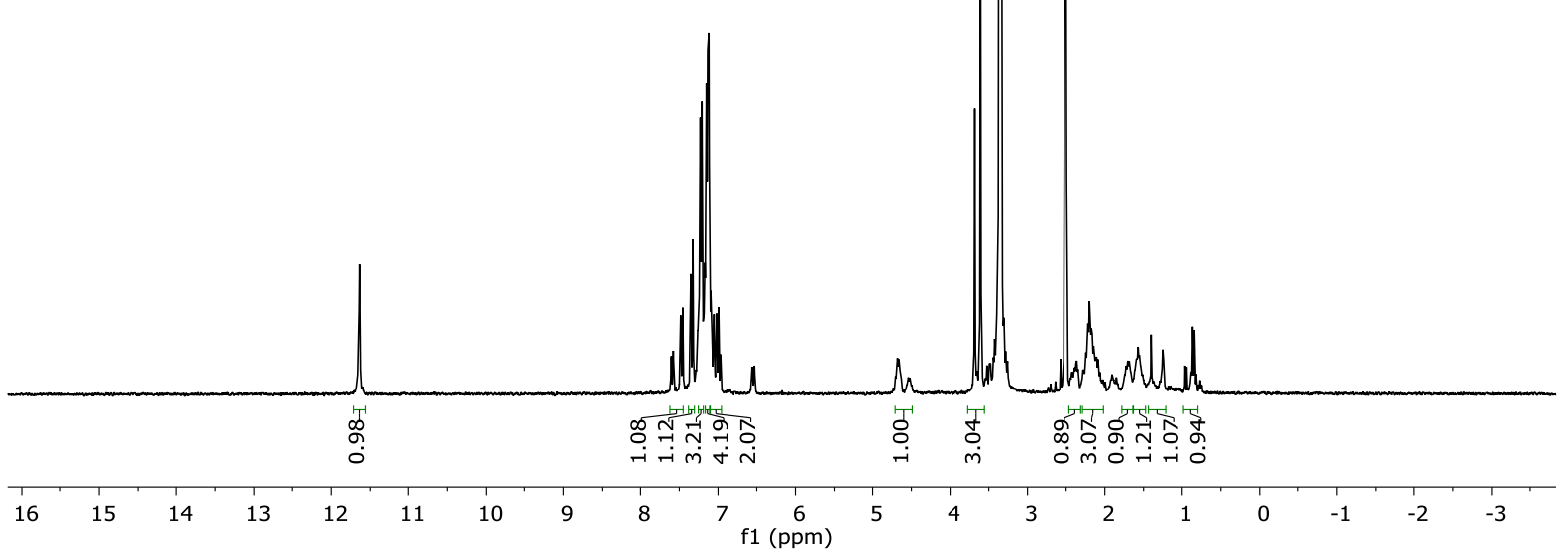

Figure S63: ${ }^{1} \mathrm{H}$ NMR spectrum of compound 7c in DMSO-d $6(300 \mathrm{MHz})$<smiles>COC(=O)C(Cc1c[nH]c2ccccc12)NC1=C([Se]c2ccccc2)C(=O)CCC1</smiles>

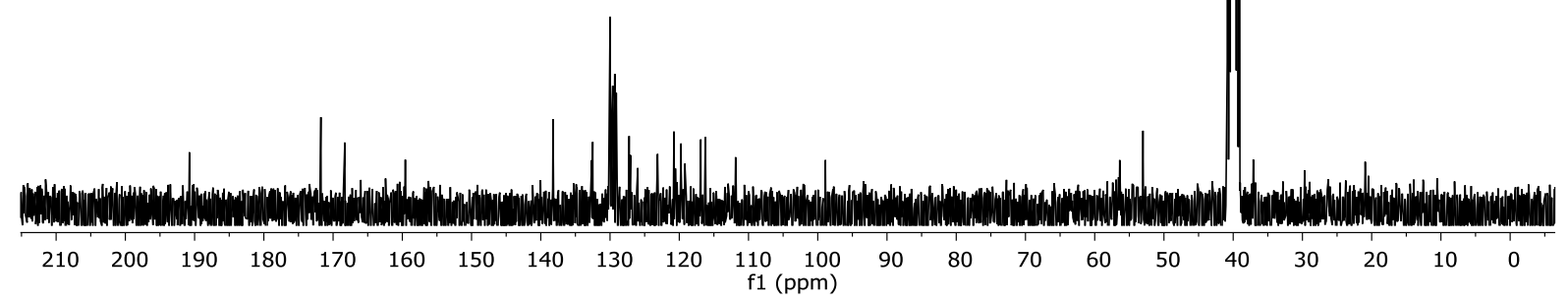

Figure S64: ${ }^{13} \mathrm{C}\left\{{ }^{1} \mathrm{H}\right\}$ NMR spectrum of compound 7c in DMSO-d $6(75 \mathrm{MHz})$ 
응

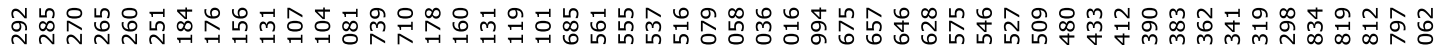

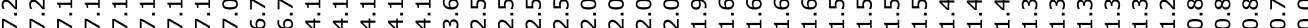
170000<smiles>COC(=O)C(CC(C)C)NC1=C([Se]c2ccccc2)C(=O)CCC1</smiles>

7d

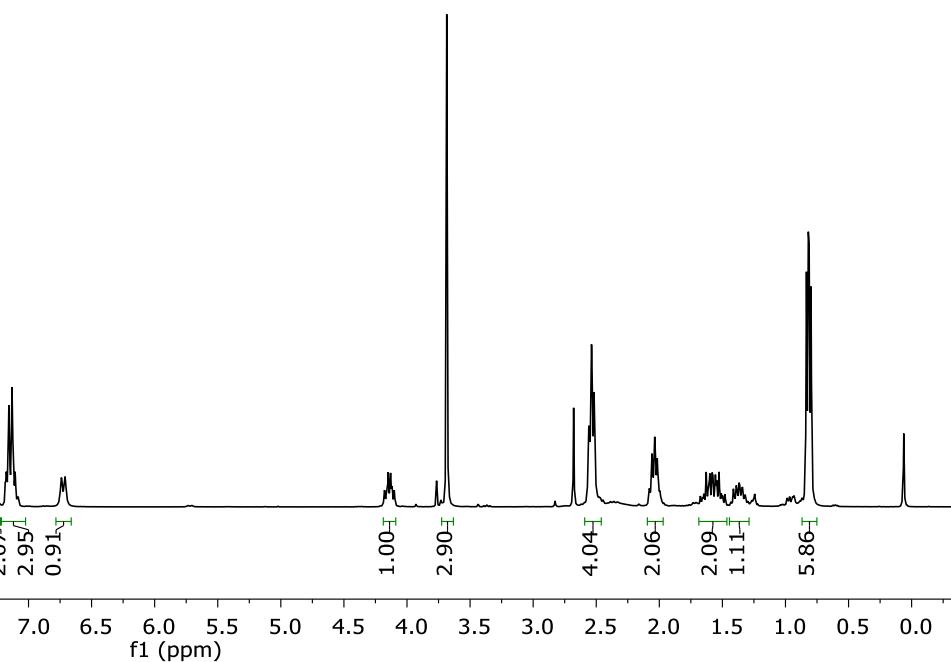

Figure S65: ${ }^{1} \mathrm{H}$ NMR spectrum of compound $7 \mathbf{d}$ in $\mathrm{CDCl}_{3}(300 \mathrm{MHz})$

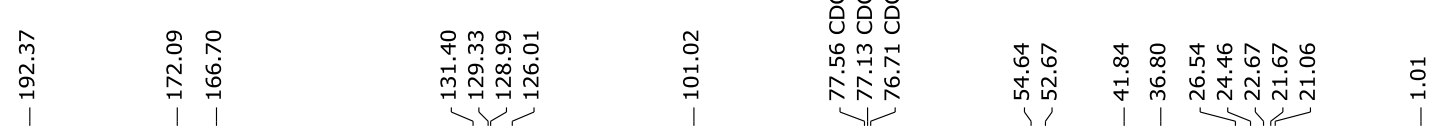<smiles>COC(=O)C(CC(C)[Ge])NC1=C([Se]c2ccccc2)C(=O)CCC1</smiles>

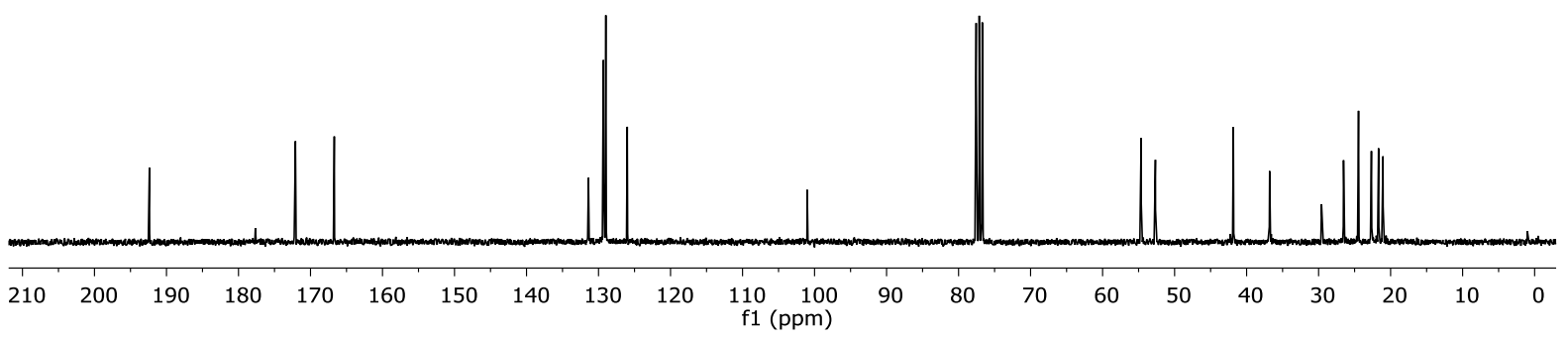

Figure S66: ${ }^{13} \mathrm{C}\left\{{ }^{1} \mathrm{H}\right\}$ NMR spectrum of compound 7d in $\mathrm{CDCl}_{3}(75 \mathrm{MHz})$ 
ֻั
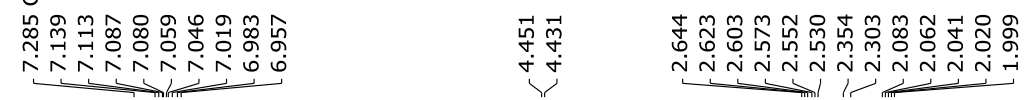<smiles>Cc1ccc(CNC2=C(Sc3ccc(C)cc3)C(=O)CCC2)cc1</smiles>

9a

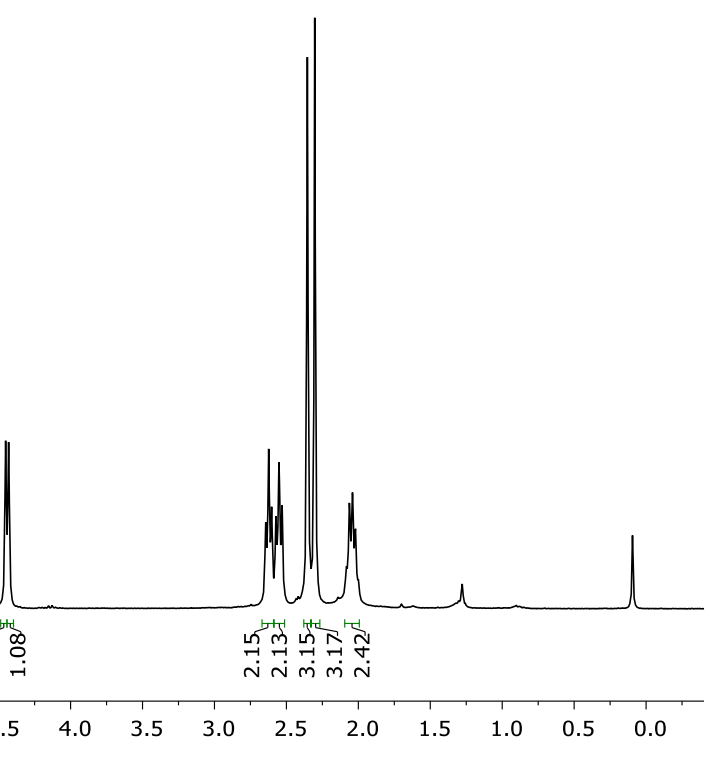

Figure S67: ${ }^{1} \mathrm{H}$ NMR spectrum of compound 9a in $\mathrm{CDCl}_{3}(300 \mathrm{MHz})$

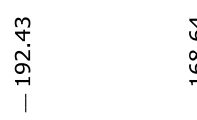

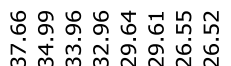

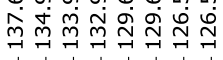

$\frac{\text { gे }}{+}$

\begin{tabular}{l}
$m$ \\
$\bar{U}$ \\
\hdashline
\end{tabular}

ㅇำ

송

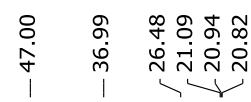<smiles>Cc1ccc(CNC2=C(Sc3ccc(C)cc3)C(=O)CCC2)cc1</smiles>

9a

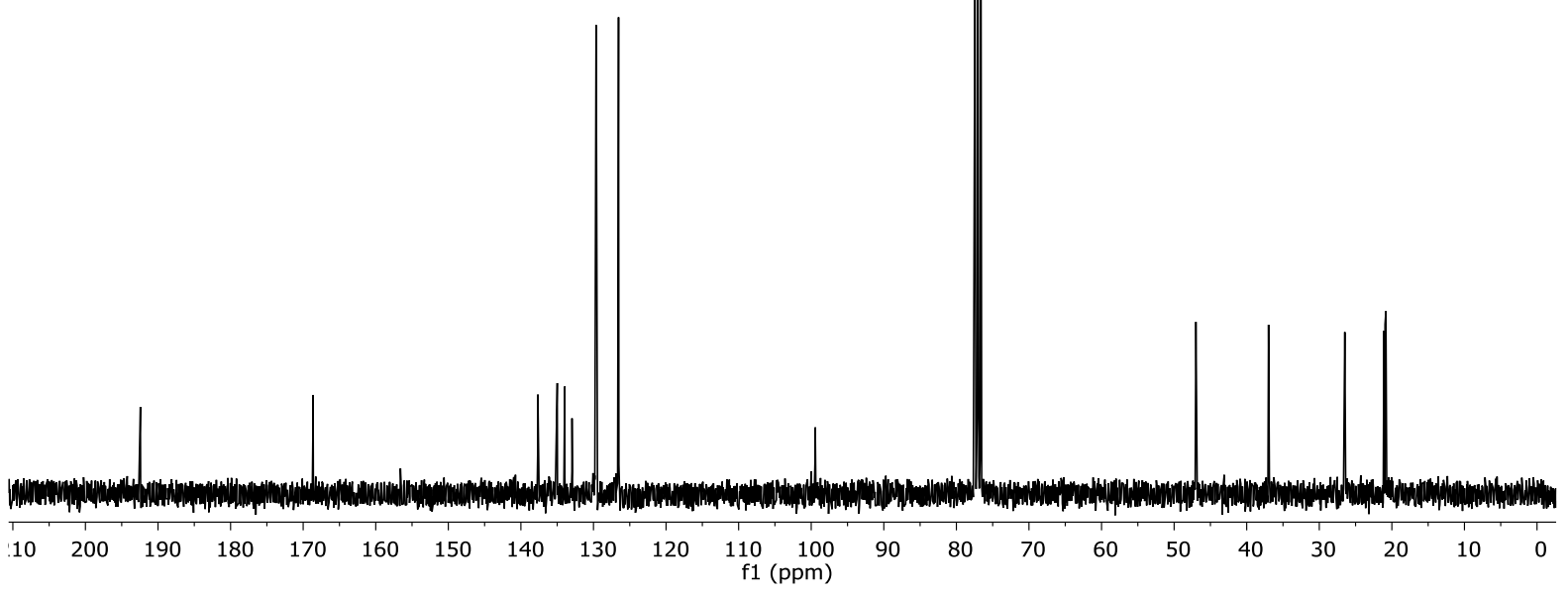

Figure S68: ${ }^{13} \mathrm{C}\left\{{ }^{1} \mathrm{H}\right\}$ NMR spectrum of compound 9a in $\mathrm{CDCl}_{3}(75 \mathrm{MHz})$ 

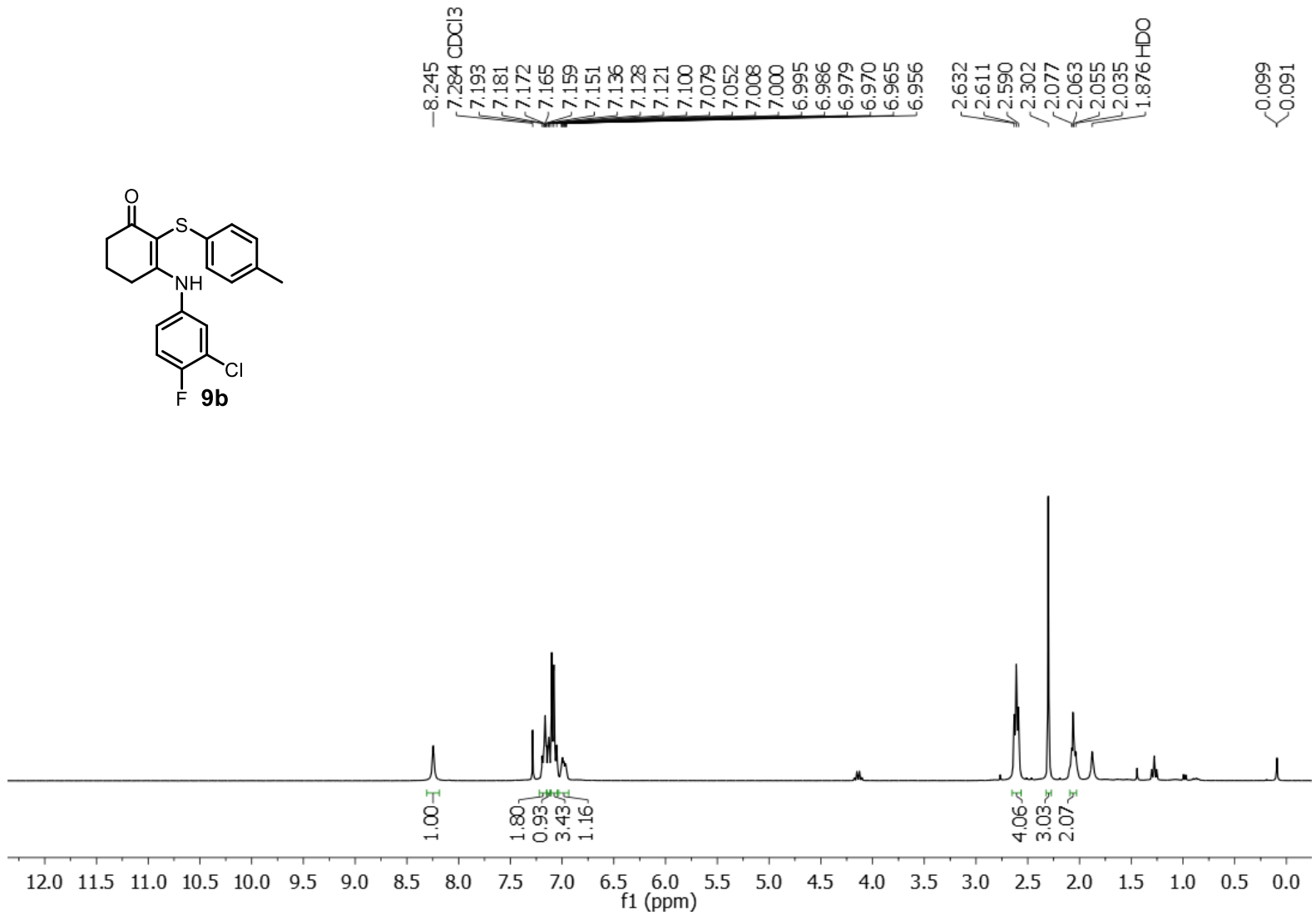

Figure S69: ${ }^{1} \mathrm{H}$ NMR spectrum of compound $9 b$ in $\mathrm{CDCl}_{3}(300 \mathrm{MHz})$

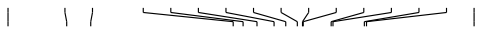<smiles>Cc1ccc(SC2=C(Nc3ccc(F)c(Cl)c3)CCCC2=O)cc1</smiles>

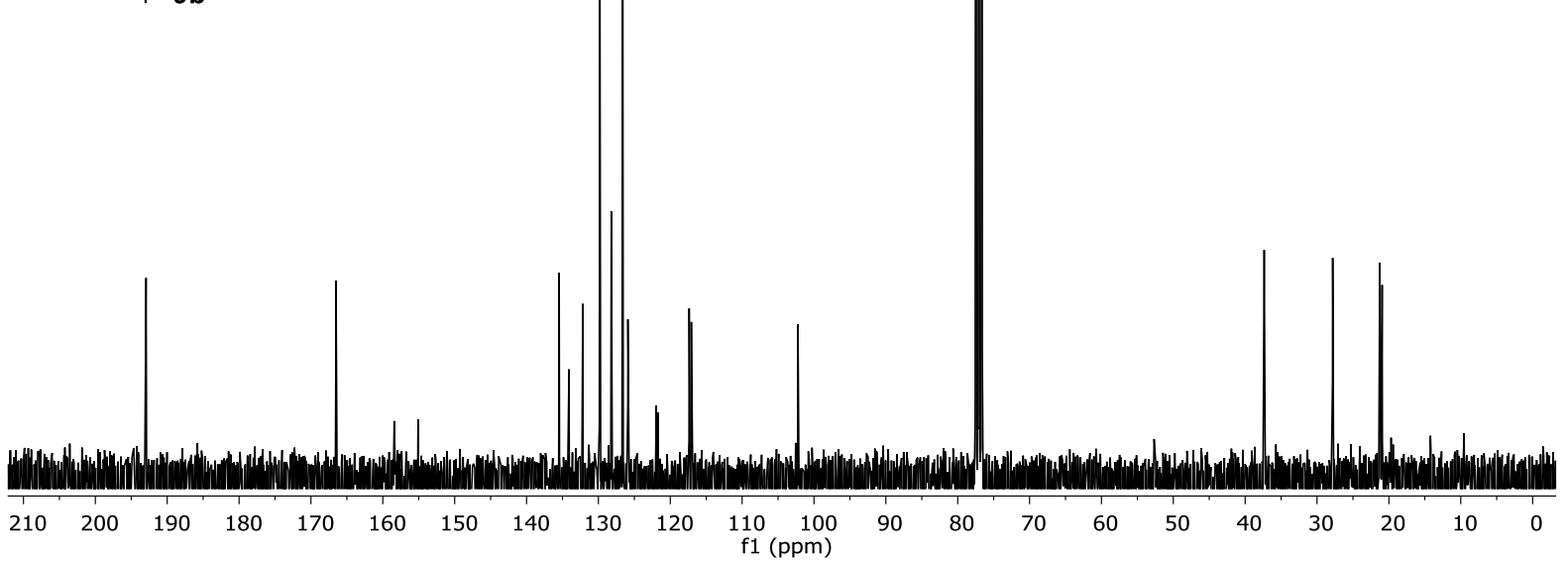

Figure S70: ${ }^{13} \mathrm{C}\left\{{ }^{1} \mathrm{H}\right\}$ NMR spectrum of compound $9 \mathbf{b}$ in $\mathrm{CDCl}_{3}(75 \mathrm{MHz})$ 
<smiles>[Mg]</smiles>

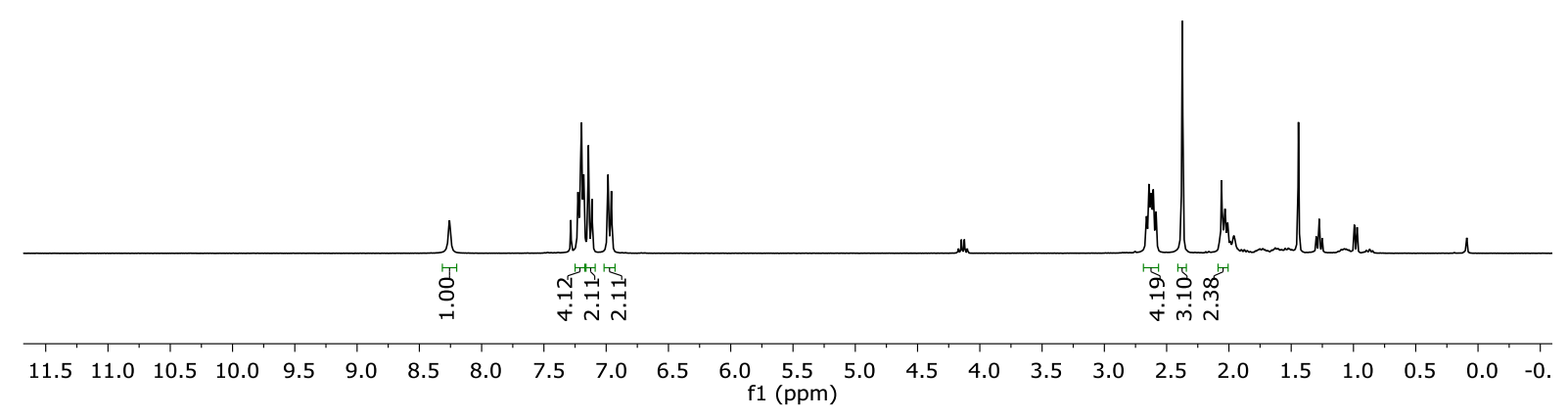

Figure S71: ${ }^{1} \mathrm{H}$ NMR spectrum of compound $9 c$ in $\mathrm{CDCl}_{3}(300 \mathrm{MHz})$

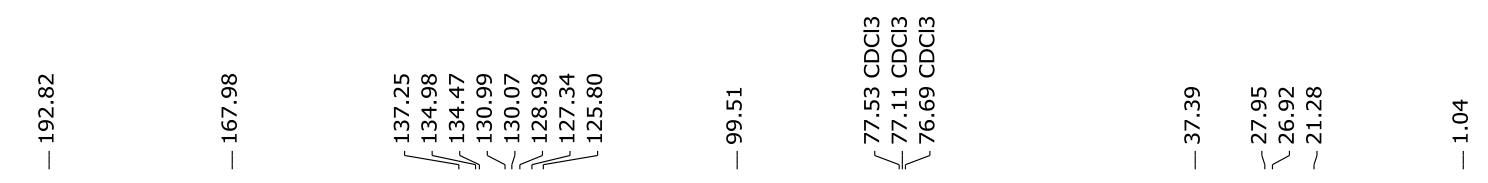<smiles>O=C1CCCC(Nc2ccc(Cl)cc2)=C1Sc1ccc(Cl)cc1</smiles>

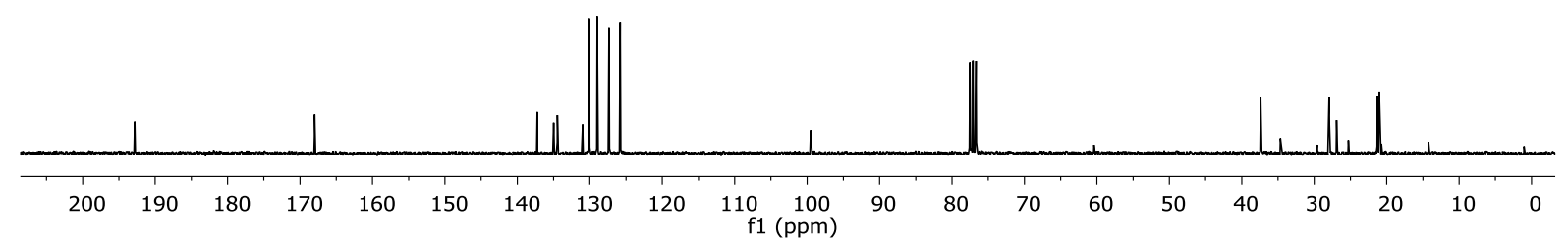

Figure S72: ${ }^{13} \mathrm{C}\left\{{ }^{1} \mathrm{H}\right\}$ NMR spectrum of compound $9 \mathrm{c}$ in $\mathrm{CDCl}_{3}(75 \mathrm{MHz})$ 


\section{$\stackrel{0}{0}$}

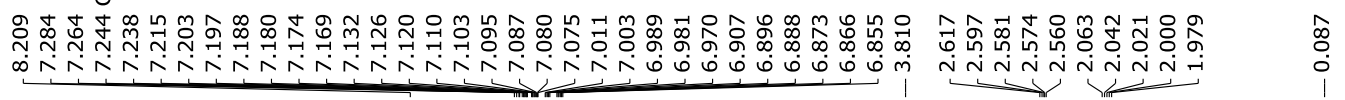<smiles>COc1ccc(NC2=C(Sc3ccccc3)C(=O)CCC2)cc1</smiles>

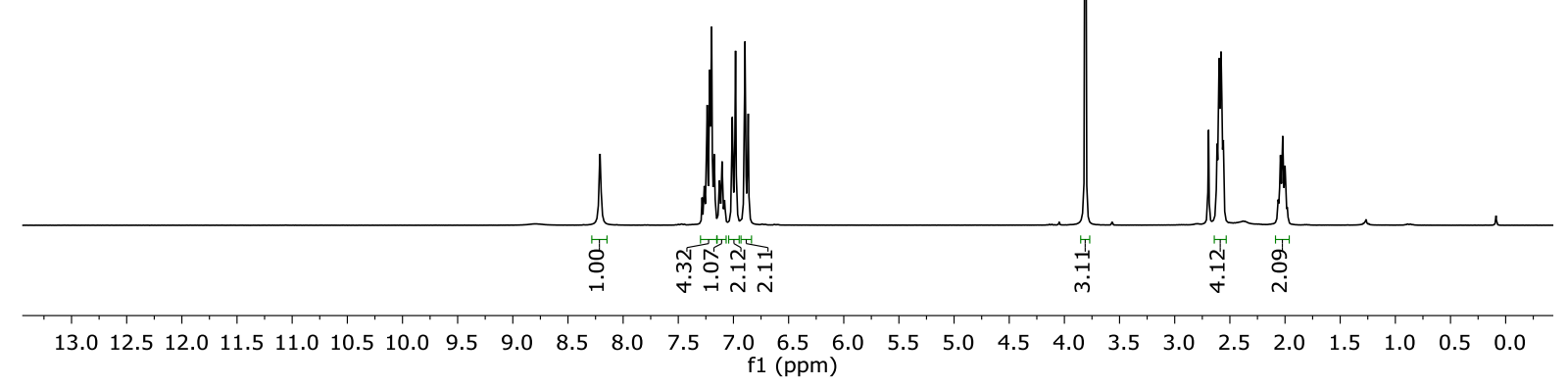

Figure S73: ${ }^{1} \mathrm{H}$ NMR spectrum of compound 9d in $\mathrm{CDCl}_{3}(300 \mathrm{MHz})$

\begin{tabular}{|c|c|c|c|c|c|c|c|c|c|}
\hline $\begin{array}{c}\text { Nू } \\
\text { ूू } \\
\text { I }\end{array}$ & $\begin{array}{l}0 \\
-1 \\
\infty \\
0 \\
-1 \\
1\end{array}$ & $\begin{array}{c}-\vec{b} \\
\infty \\
\infty \\
\stackrel{0}{-1} \\
\mid\end{array}$ & 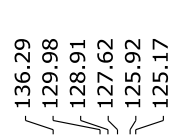 & $\begin{array}{c}\hat{n} \\
\stackrel{+}{-} \\
\stackrel{-1}{1}\end{array}$ & $\begin{array}{l}\text { ㅇ. } \\
\text { gे } \\
\text { i }\end{array}$ & 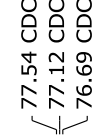 & 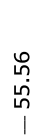 & 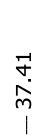 & 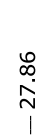 \\
\hline
\end{tabular}<smiles>COc1ccc(NC2=C(Sc3ccccc3)C(=O)CCC2)cc1</smiles>

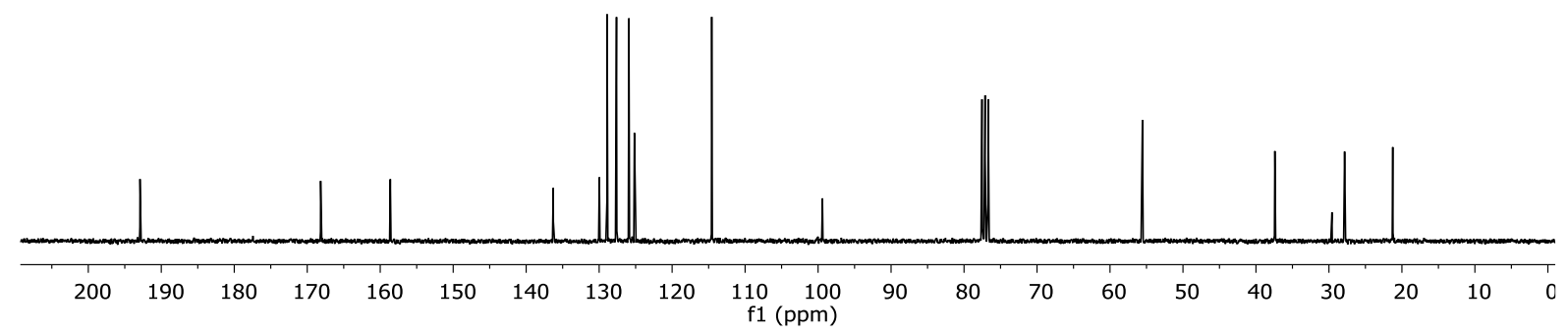

Figure S74: ${ }^{13} \mathrm{C}\left\{{ }^{1} \mathrm{H}\right\}$ NMR spectrum of compound $9 d$ in $\mathrm{CDCl}_{3}(75 \mathrm{MHz})$ 
商

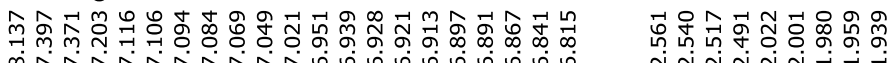

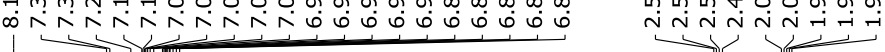<smiles>O=C1CCCC2=C(Sc3ccccc3Br)Nc3ccc(F)c(Cl)c3C12</smiles>

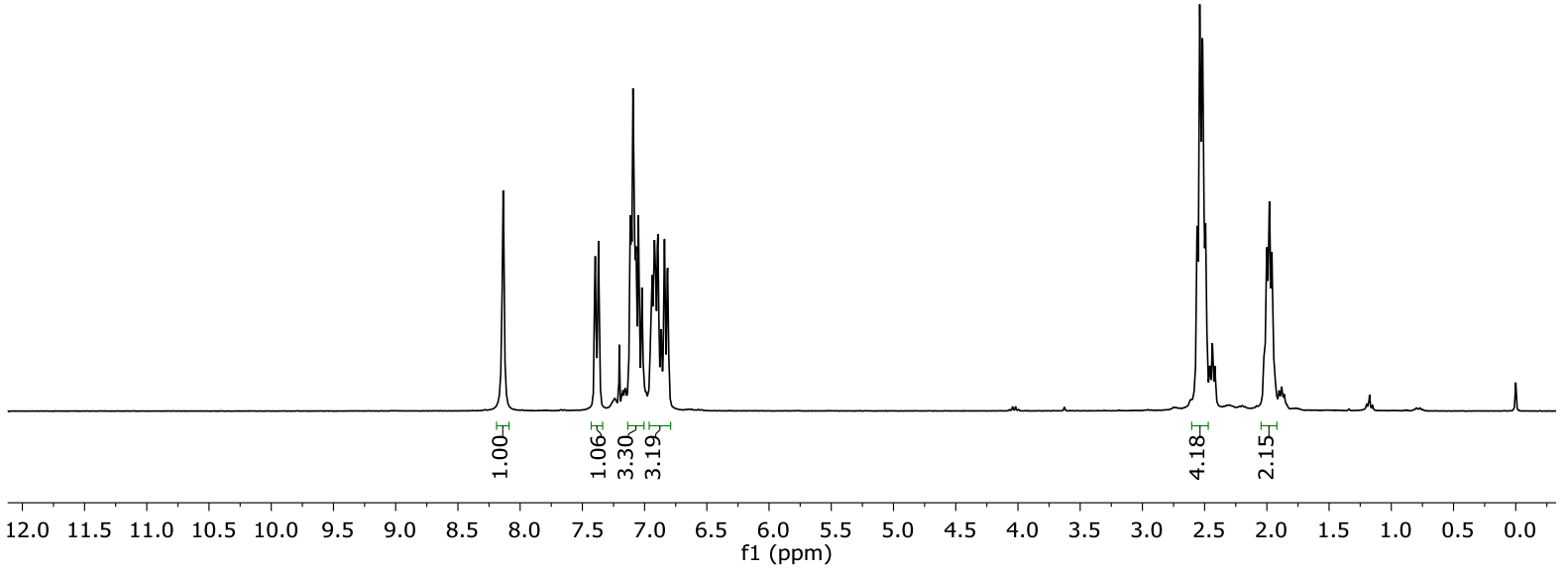

Figure S75: ${ }^{1} \mathrm{H}$ NMR spectrum of compound $9 e$ in $\mathrm{CDCl}_{3}(300 \mathrm{MHz})$

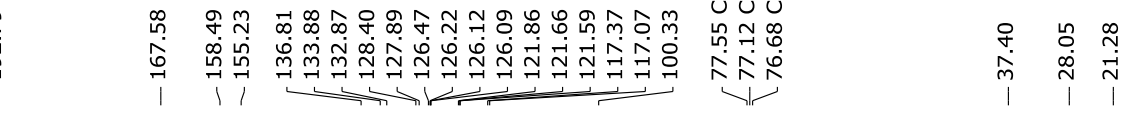<smiles>O=C1CCCC(Nc2cccc(F)c2)=C1Sc1ccccc1Br</smiles>

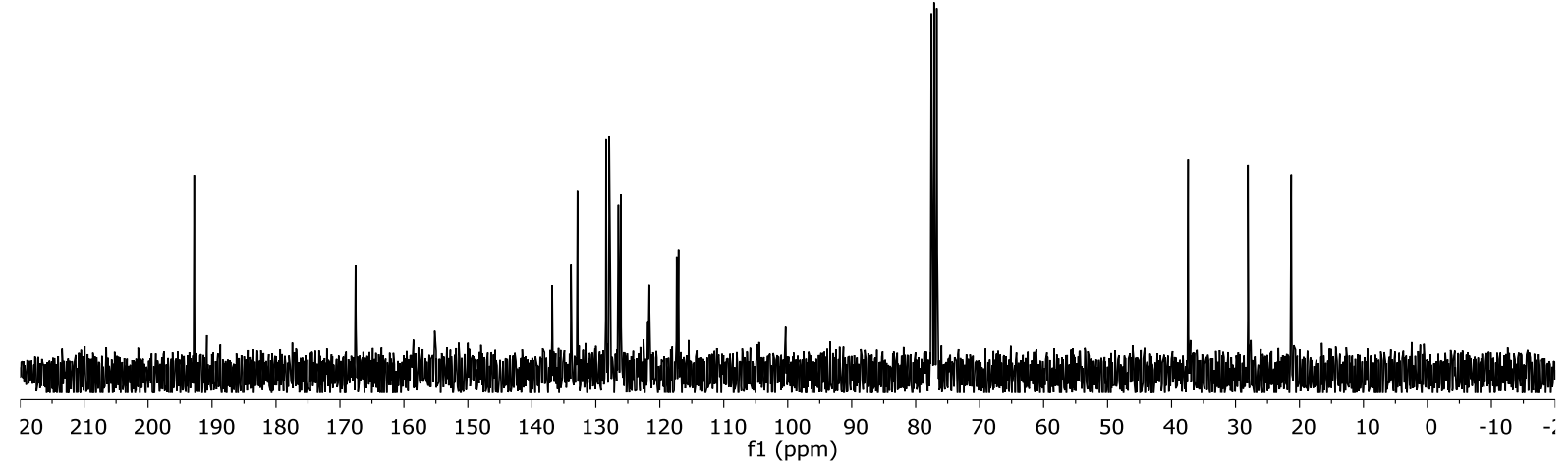

Figure S76: ${ }^{13} \mathrm{C}\left\{{ }^{1} \mathrm{H}\right\}$ NMR spectrum of compound $9 e$ in $\mathrm{CDCl}_{3}(75 \mathrm{MHz})$ 


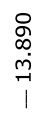

Nv

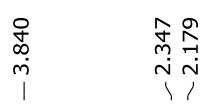

$\ddot{0}$
o
i
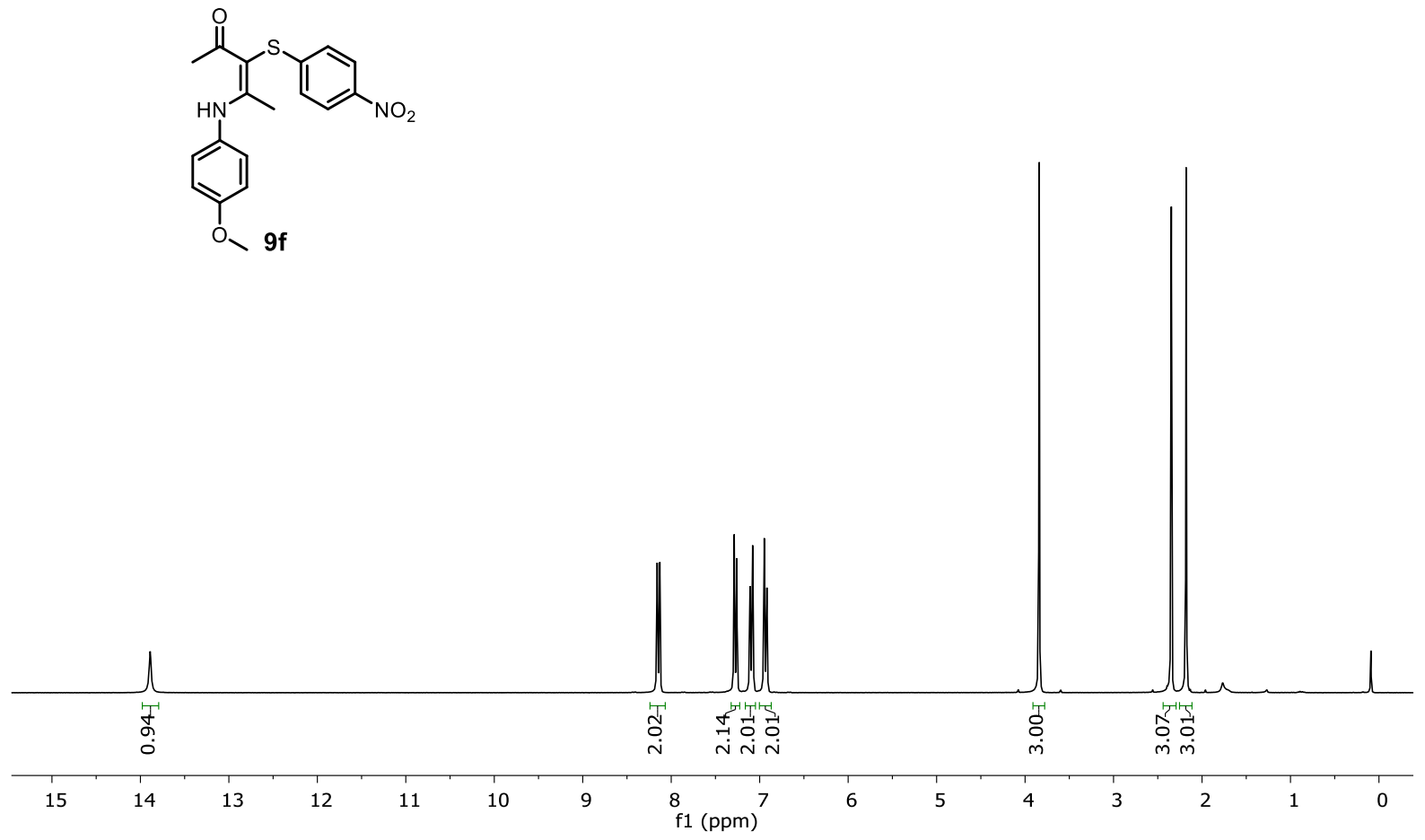

Figure S77: ${ }^{1} \mathrm{H}$ NMR spectrum of compound $9 f$ in $\mathrm{CDCl}_{3}(300 \mathrm{MHz})$

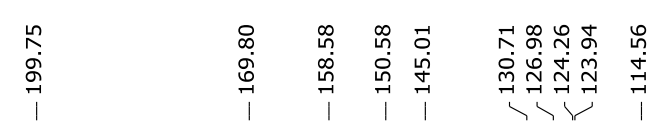

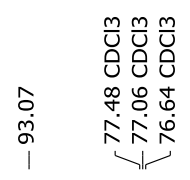

華

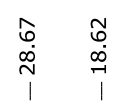

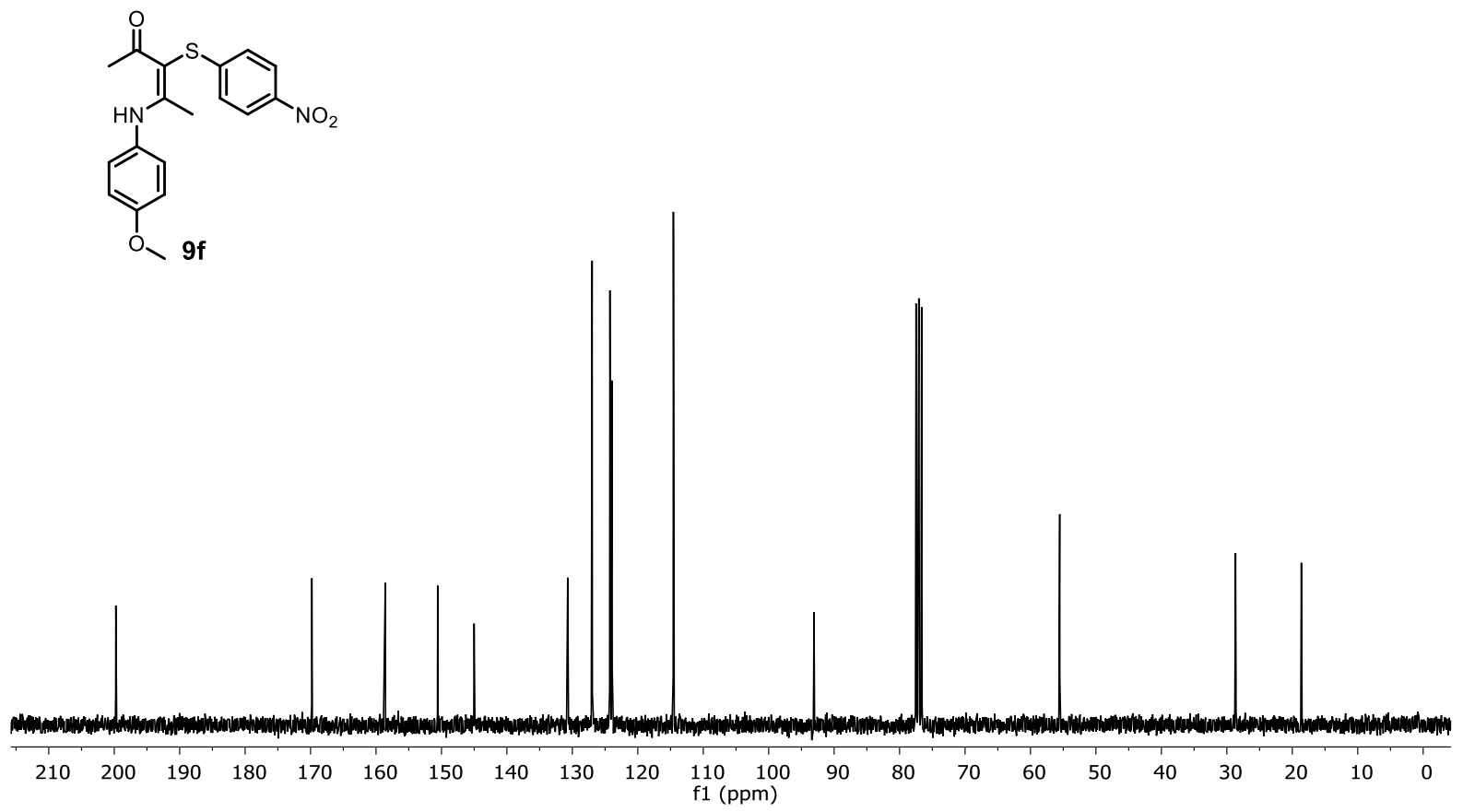

Figure S78: ${ }^{13} \mathrm{C}\left\{{ }^{1} \mathrm{H}\right\}$ NMR spectrum of compound 9 f in $\mathrm{CDCl}_{3}(75 \mathrm{MHz})$

S40 

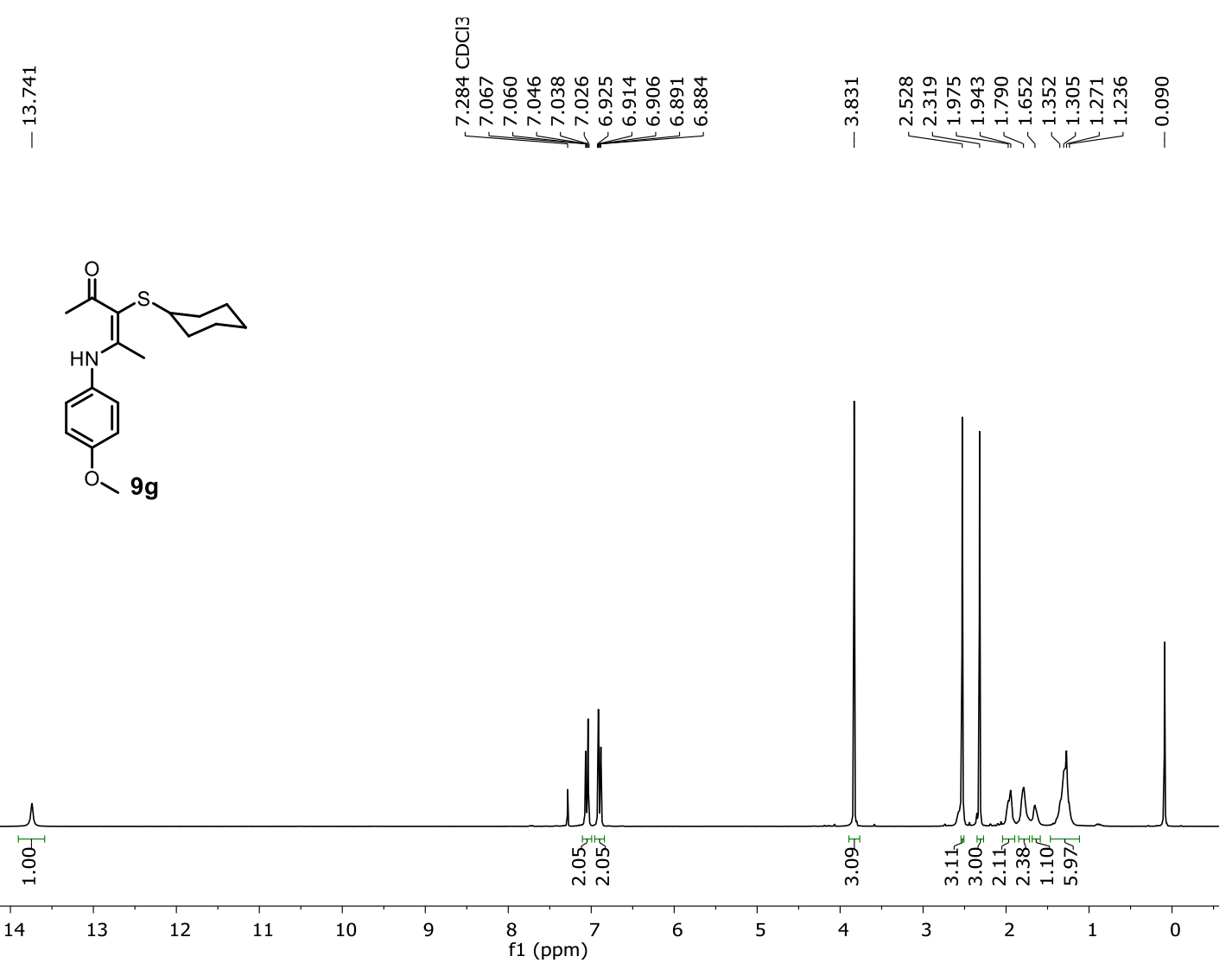

Figure S79: ${ }^{1} \mathrm{H}$ NMR spectrum of compound $9 \mathrm{~g}$ in $\mathrm{CDCl}_{3}(300 \mathrm{MHz})$
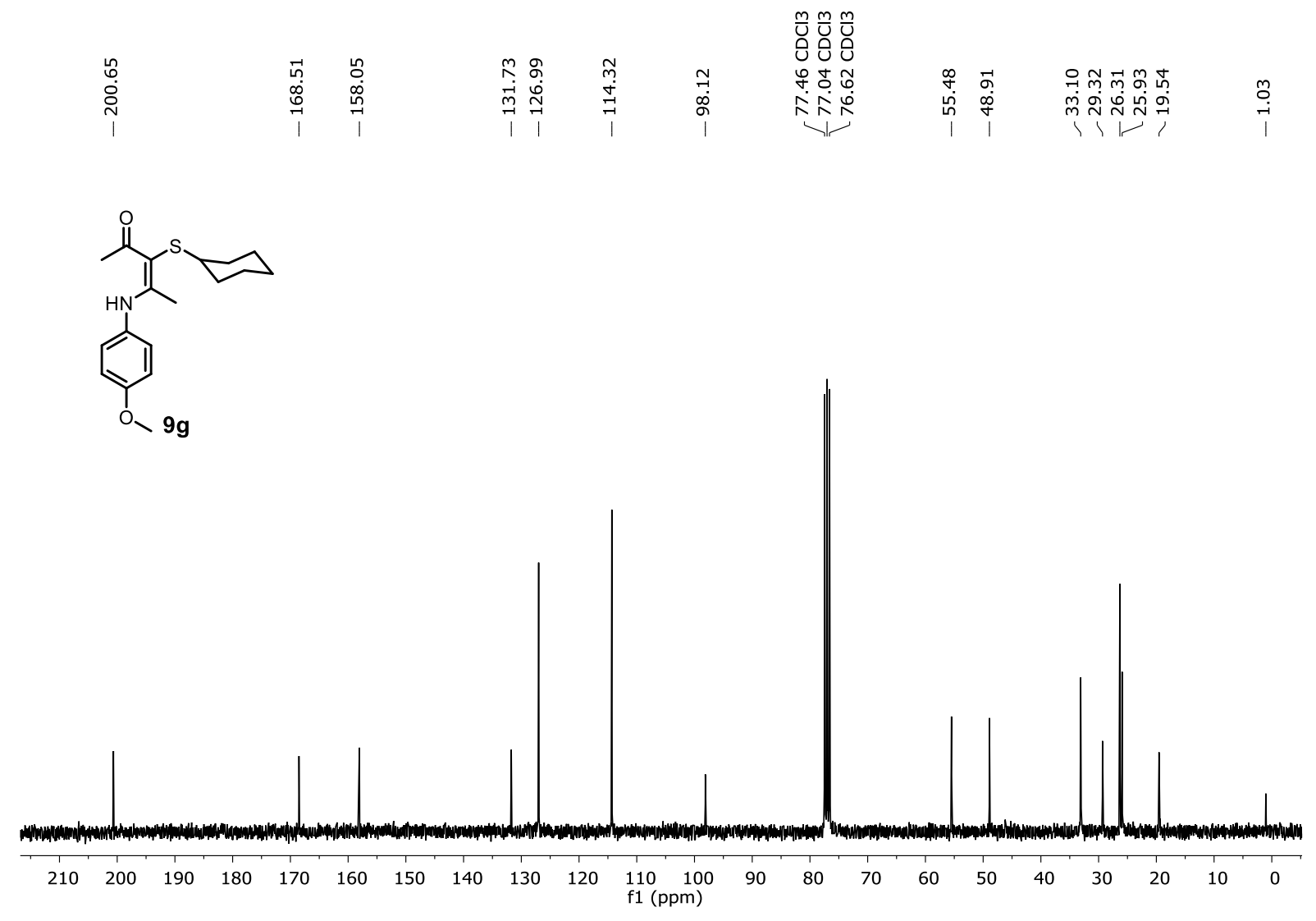

Figure S80: ${ }^{13} \mathrm{C}\left\{{ }^{1} \mathrm{H}\right\}$ NMR spectrum of compound 9 g in $\mathrm{CDCl}_{3}(75 \mathrm{MHz})$ 
<smiles>O=C1CCCC(Cc2ccccc2)=C1Sc1nc2ccccc2s1</smiles>

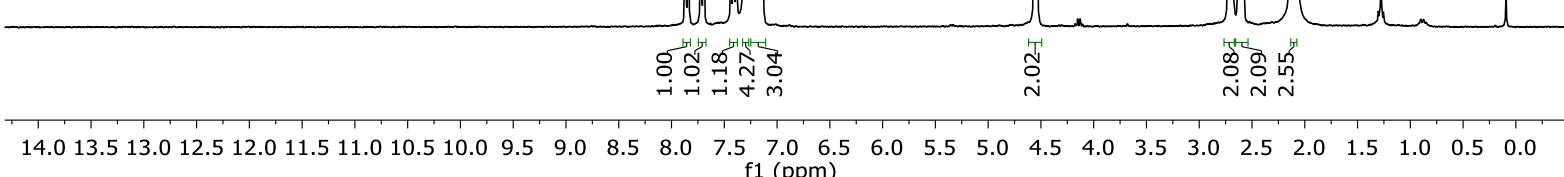

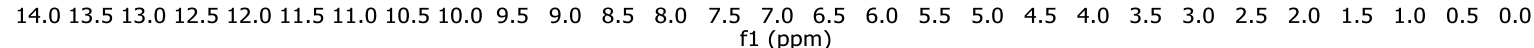

Figure S81: ${ }^{1} \mathrm{H}$ NMR spectrum of compound 9h in $\mathrm{CDCl}_{3}(300 \mathrm{MHz})$

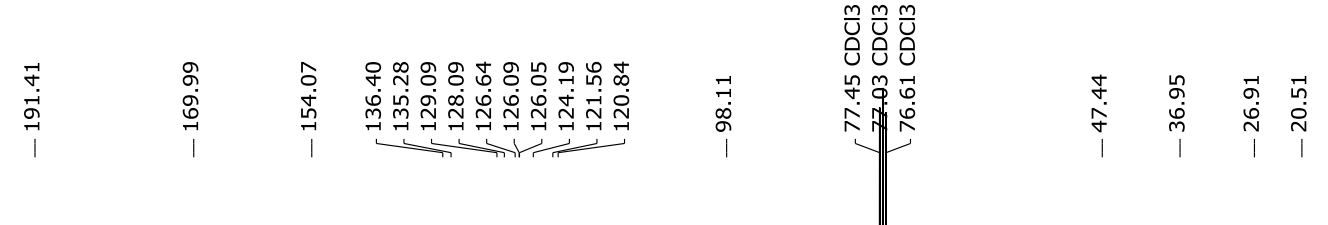<smiles>O=C1CCCC2=C1SC1=Nc3ccccc3S1=C2</smiles>

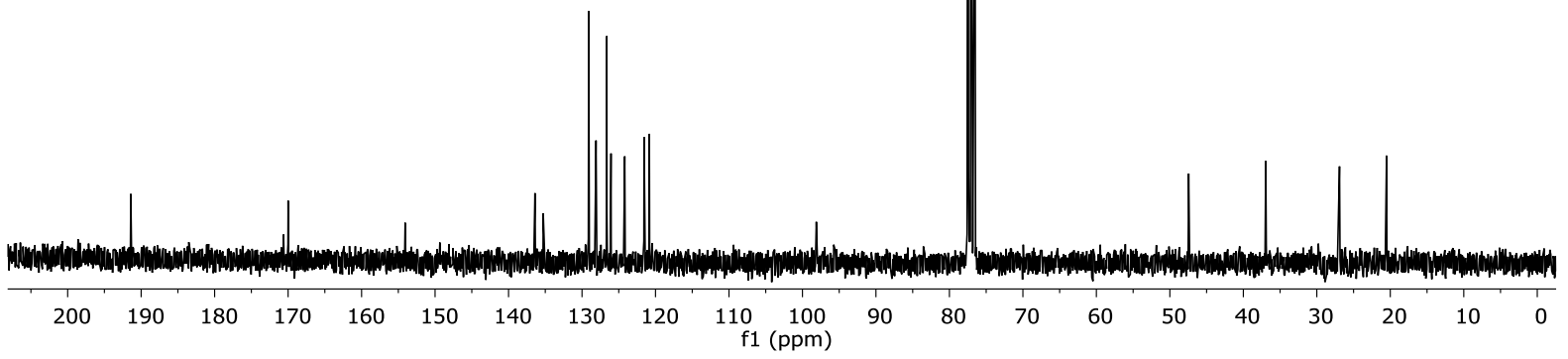

Figure S82: ${ }^{13} \mathrm{C}\left\{{ }^{1} \mathrm{H}\right\}$ NMR spectrum of compound $\mathbf{9 h}$ in $\mathrm{CDCl}_{3}(75 \mathrm{MHz})$ 

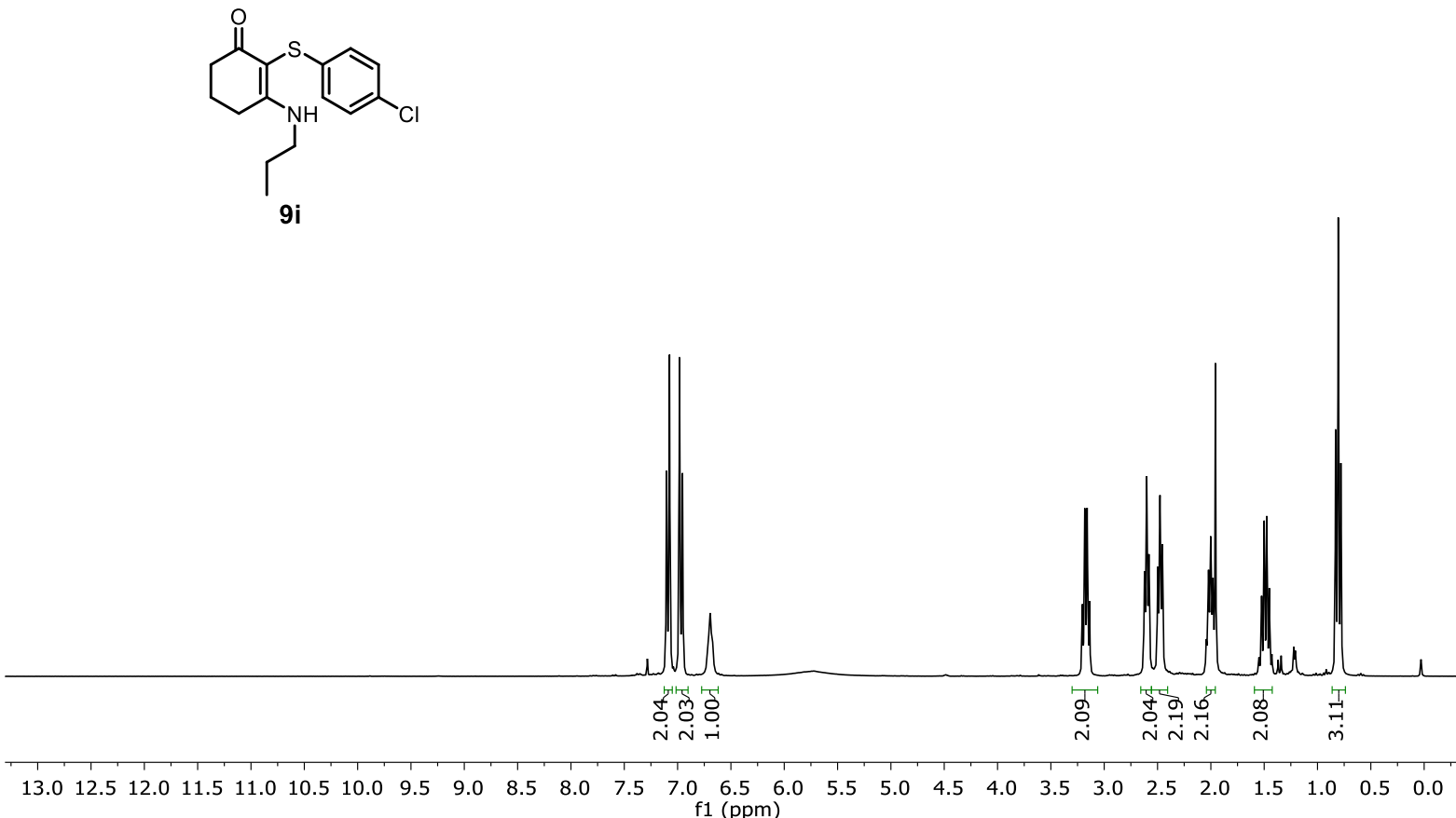

Figure S83: ${ }^{1} \mathrm{H}$ NMR spectrum of compound $9 \mathbf{i}$ in $\mathrm{CDCl}_{3}(300 \mathrm{MHz})$<smiles>CCCNC1=C(Sc2ccc(C)cc2)C(=O)CCC1</smiles>

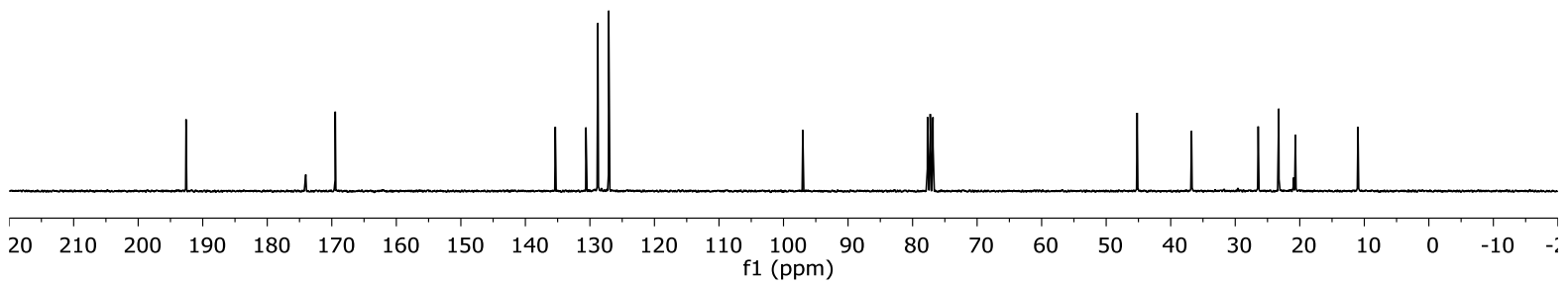

Figure S84: ${ }^{13} \mathrm{C}\left\{{ }^{1} \mathrm{H}\right\}$ NMR spectrum of compound 9i in $\mathrm{CDCl}_{3}(75 \mathrm{MHz})$ 
రับ

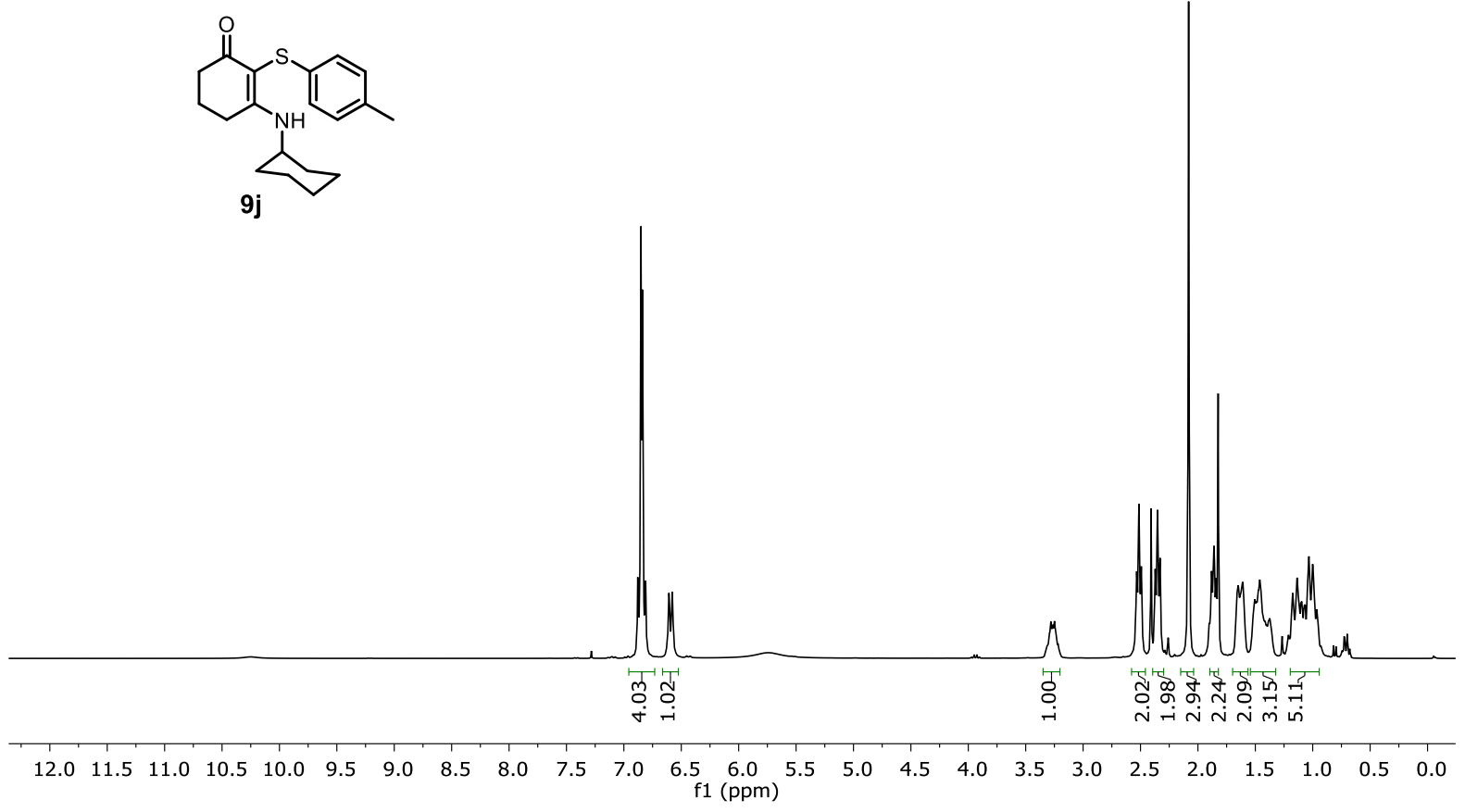

Figure S85: ${ }^{1} \mathrm{H}$ NMR spectrum of compound $9 \mathbf{j}$ in $\mathrm{CDCl}_{3}(300 \mathrm{MHz})$

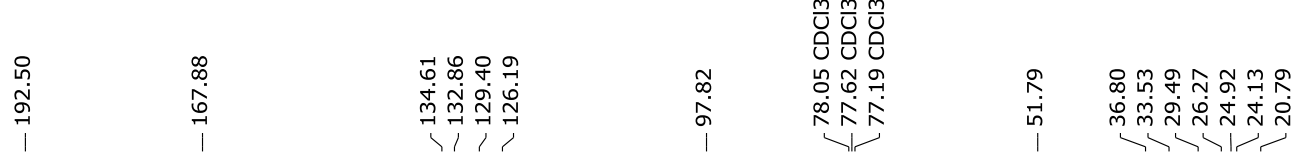<smiles>CCCCC(C)NC1=C(Sc2ccc(C)cc2)C(=O)CCC1</smiles>

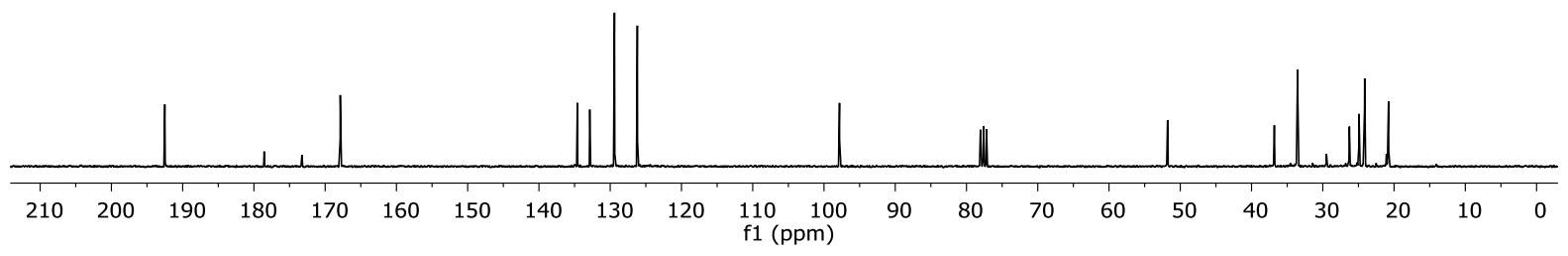

Figure S86: ${ }^{13} \mathrm{C}\left\{{ }^{1} \mathrm{H}\right\}$ NMR spectrum of compound $9 \mathbf{j}$ in $\mathrm{CDCl}_{3}(75 \mathrm{MHz})$ 
<smiles>Cc1ccc(NC2=C([Se]c3ccccc3)C(=O)CC(C)(C)C2)cc1</smiles>

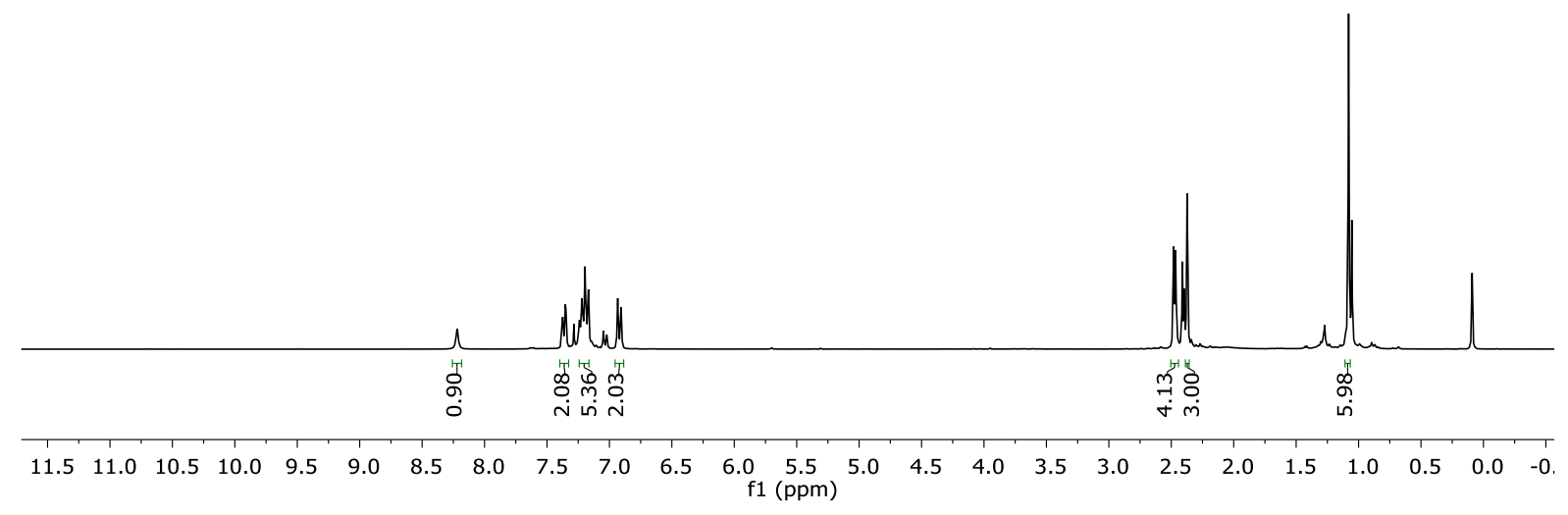

Figure S87: ${ }^{1} \mathrm{H}$ NMR spectrum of compound 9k in $\mathrm{CDCl}_{3}(300 \mathrm{MHz})$<smiles>Cc1ccc(NC2=C([Se]c3ccccc3)C(=O)CC(C)(C)C2)cc1</smiles>

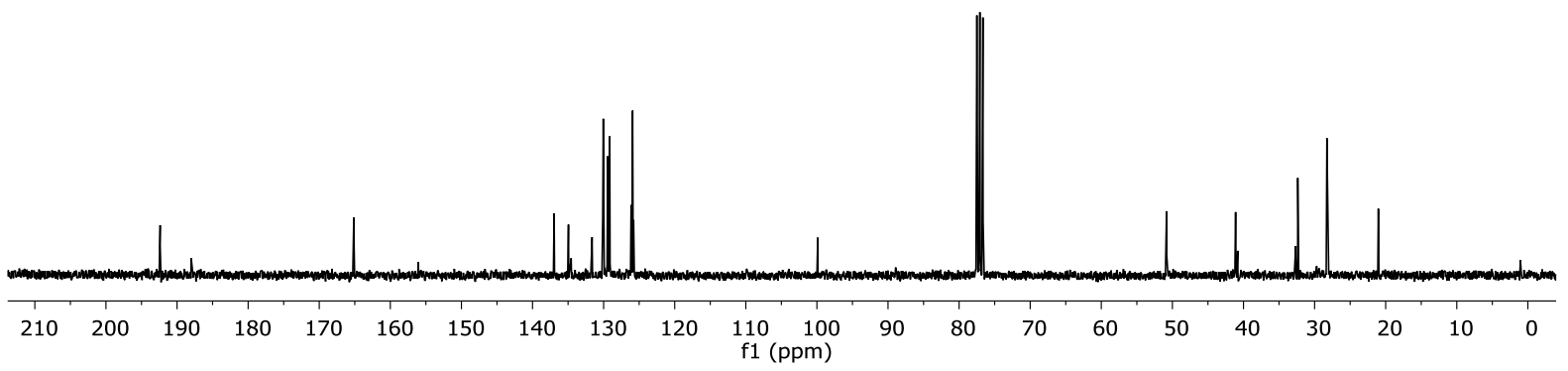

Figure S88: ${ }^{13} \mathrm{C}\left\{{ }^{1} \mathrm{H}\right\}$ NMR spectrum of compound 9k in $\mathrm{CDCl}_{3}(75 \mathrm{MHz})$ 


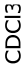

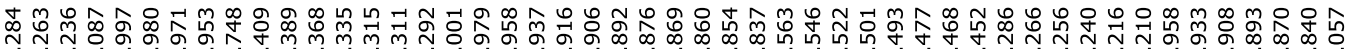

垈

이인

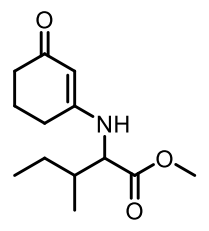

$3 a$

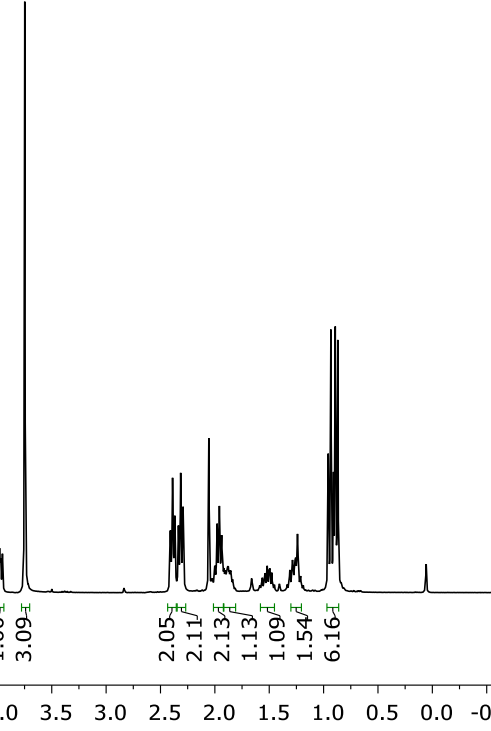

$\begin{array}{lllllllllllllllllllllllllllllllll}13.5 & 13.0 & 12.5 & 12.0 & 11.5 & 11.0 & 10.5 & 10.0 & 9.5 & 9.0 & 8.5 & 8.0 & 7.5 & 7.0 & 6.5 & 6.0 & 5.5 & 5.0 & 4.5 & 4.0 & 3.5 & 3.0 & 2.5 & 2.0 & 1.5 & 1.0 & 0.5 & 0.0 & -0\end{array}$

Figure S89: ${ }^{1} \mathrm{H}$ NMR spectrum of compound 3a in $\mathrm{CDCl}_{3}(300 \mathrm{MHz})$

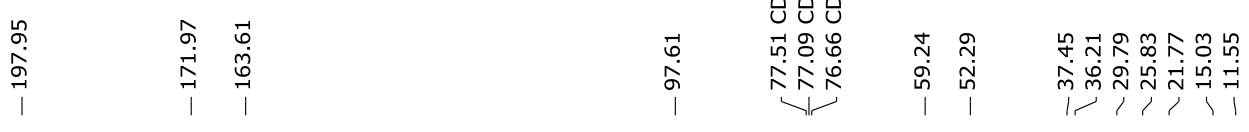

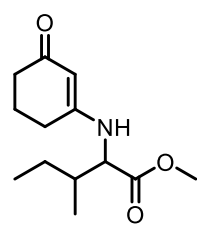

3a

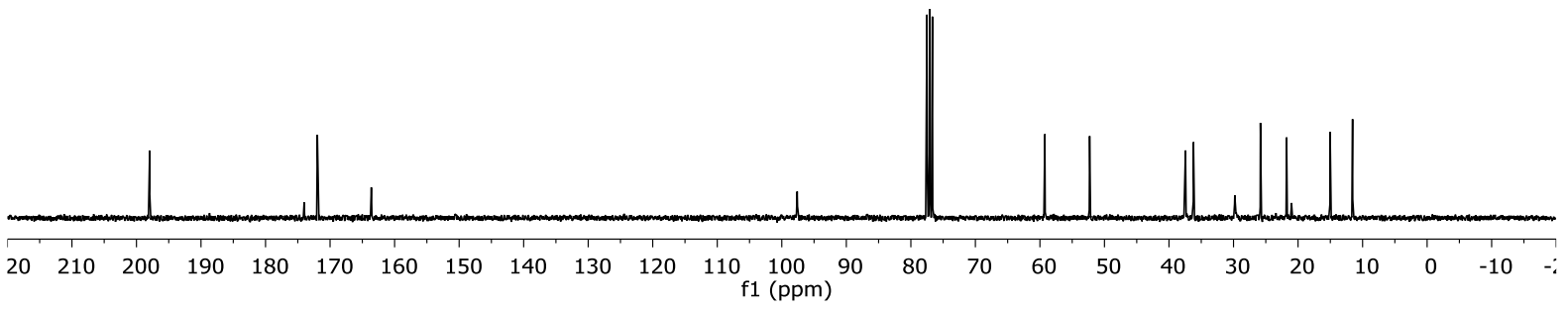

Figure S90: ${ }^{13} \mathrm{C}\left\{{ }^{1} \mathrm{H}\right\}$ NMR spectrum of compound 3a in $\mathrm{CDCl}_{3}(75 \mathrm{MHz})$ 
商

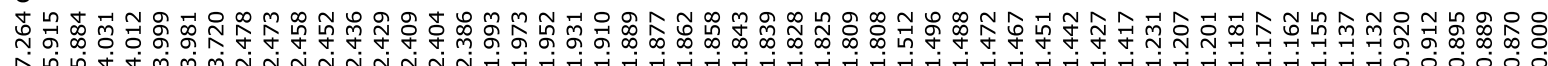

ํ.

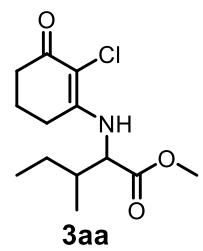

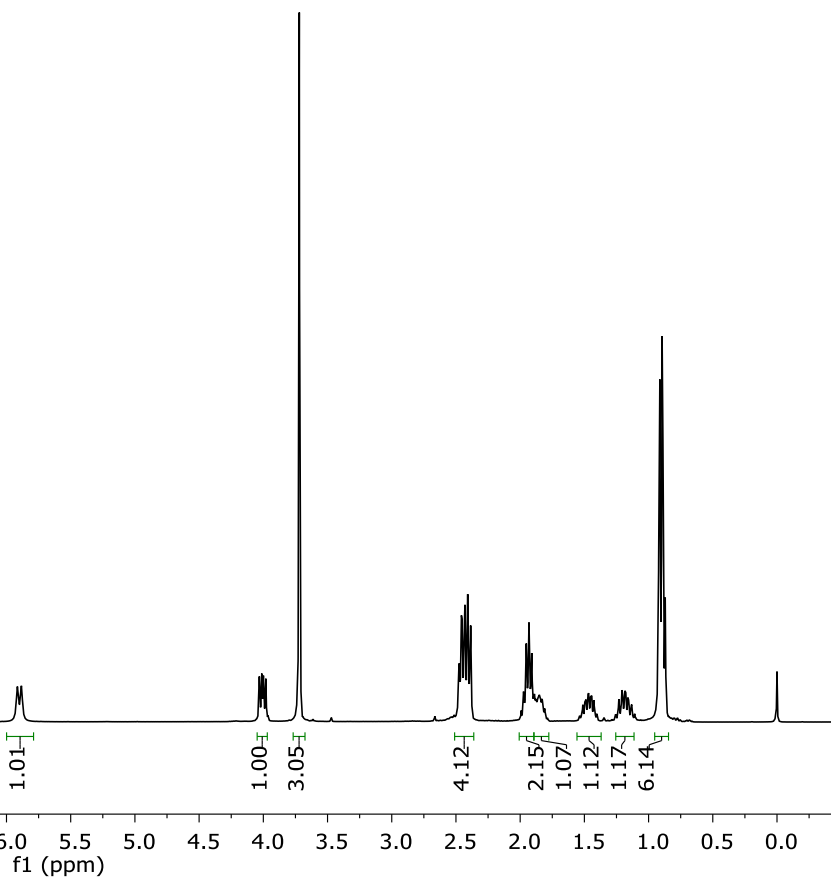

Figure S91: ${ }^{1} \mathrm{H}$ NMR spectrum of compound 3aa in $\mathrm{CDCl}_{3}(300 \mathrm{MHz})$

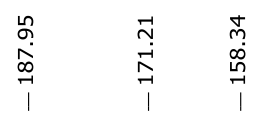
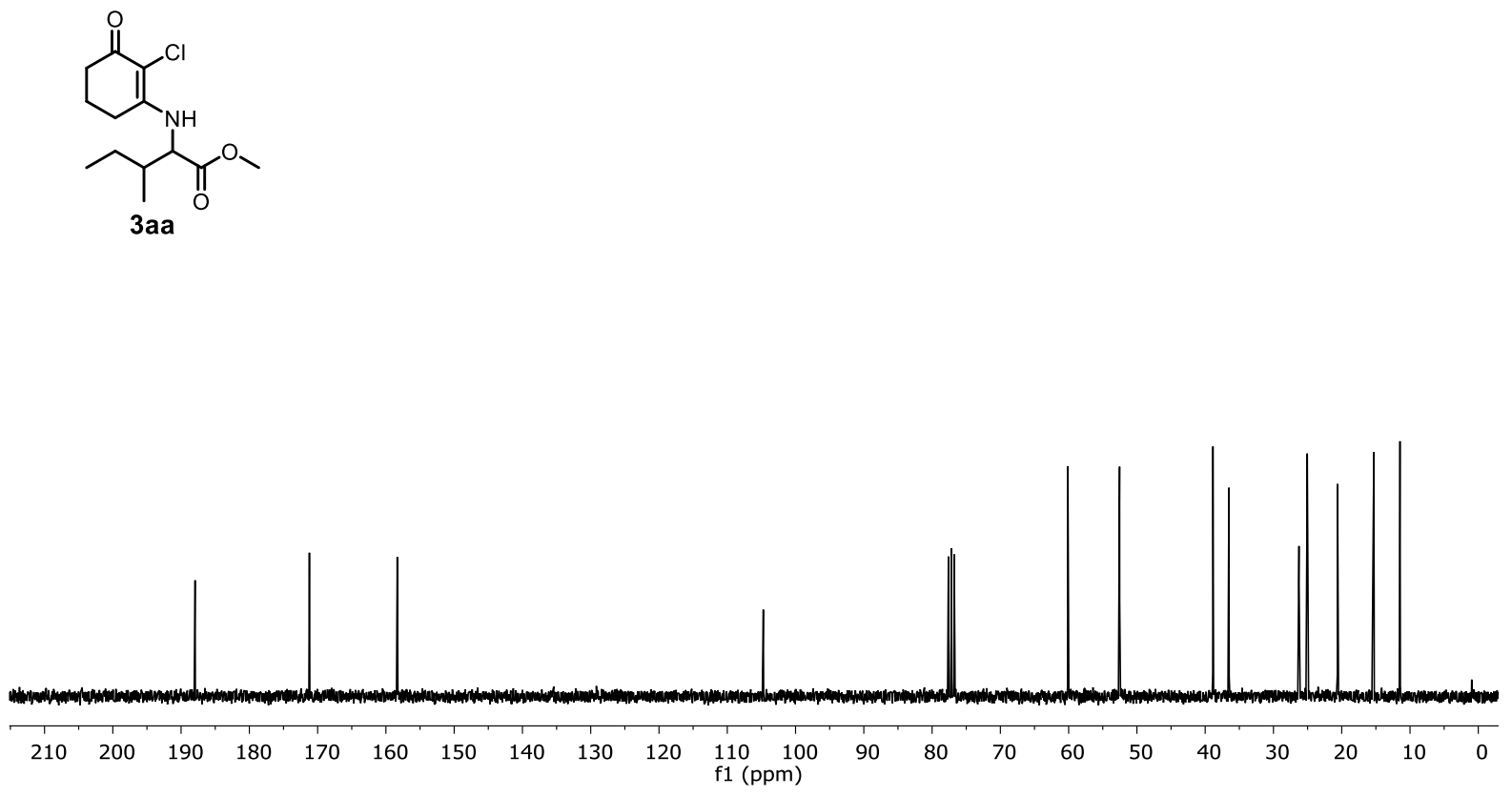

Figure S92: ${ }^{13} \mathrm{C}\left\{{ }^{1} \mathrm{H}\right\}$ NMR spectrum of compound $\mathbf{3 a a}$ in $\mathrm{CDCl}_{3}(75 \mathrm{MHz})$ 


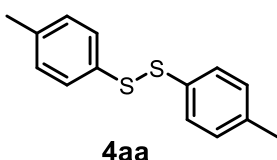

$4 a a$

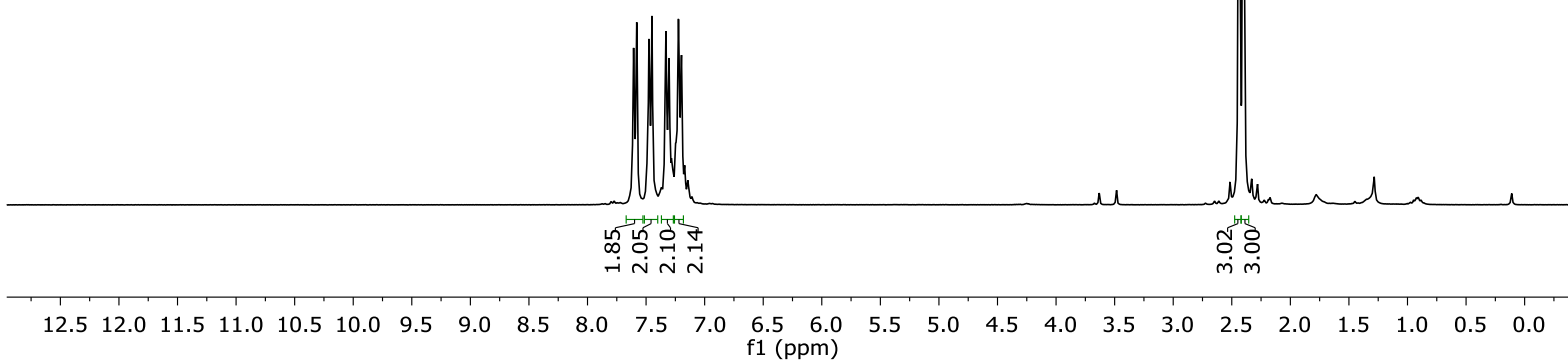

Figure S93: ${ }^{1} \mathrm{H}$ NMR spectrum of compound 4aa in $\mathrm{CDCl}_{3}(300 \mathrm{MHz})$

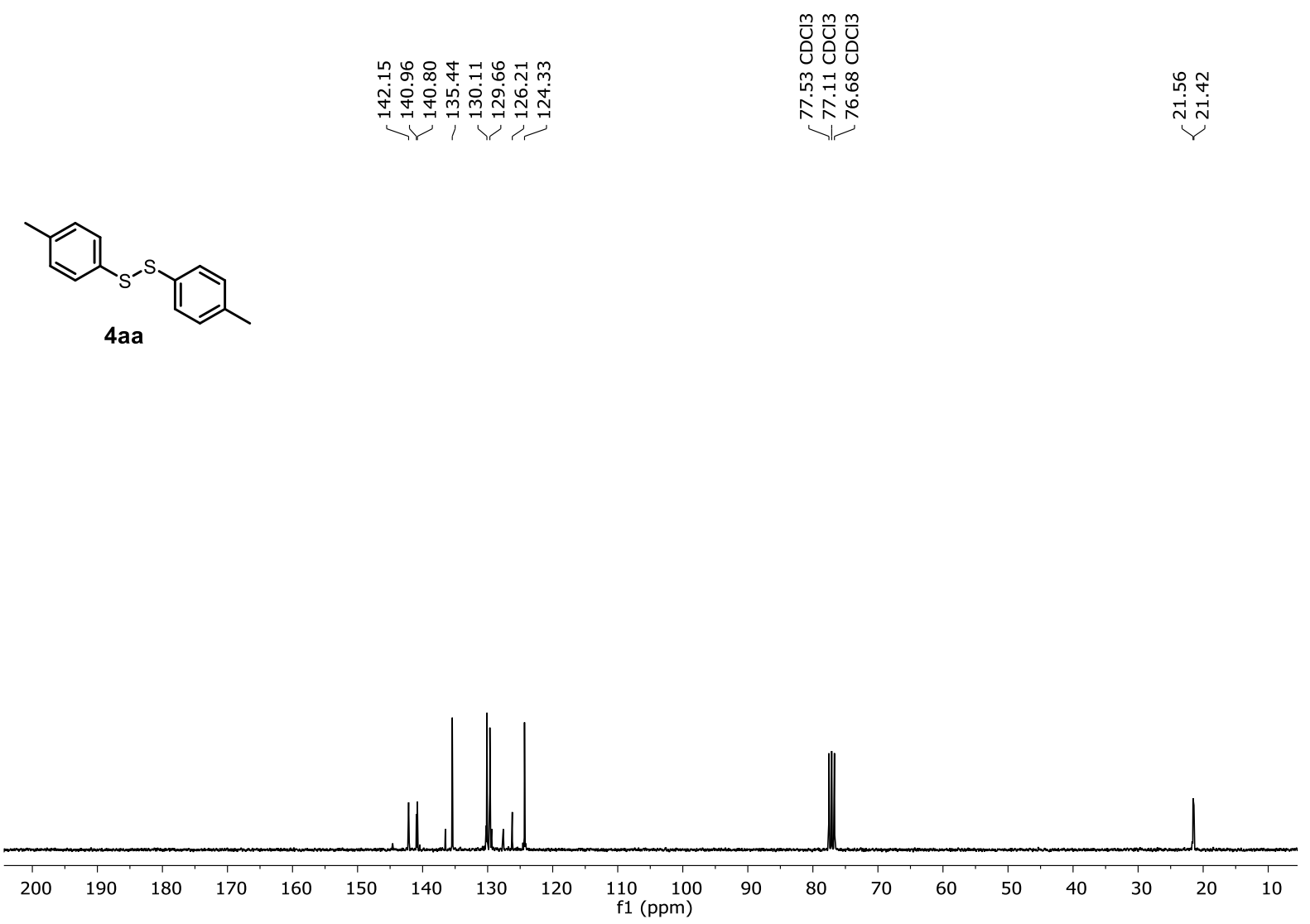

Figure S94: ${ }^{13} \mathrm{C}\left\{{ }^{1} \mathrm{H}\right\}$ NMR spectrum of compound $\mathbf{4 a a}$ in $\mathrm{CDCl}_{3}(75 \mathrm{MHz})$ 


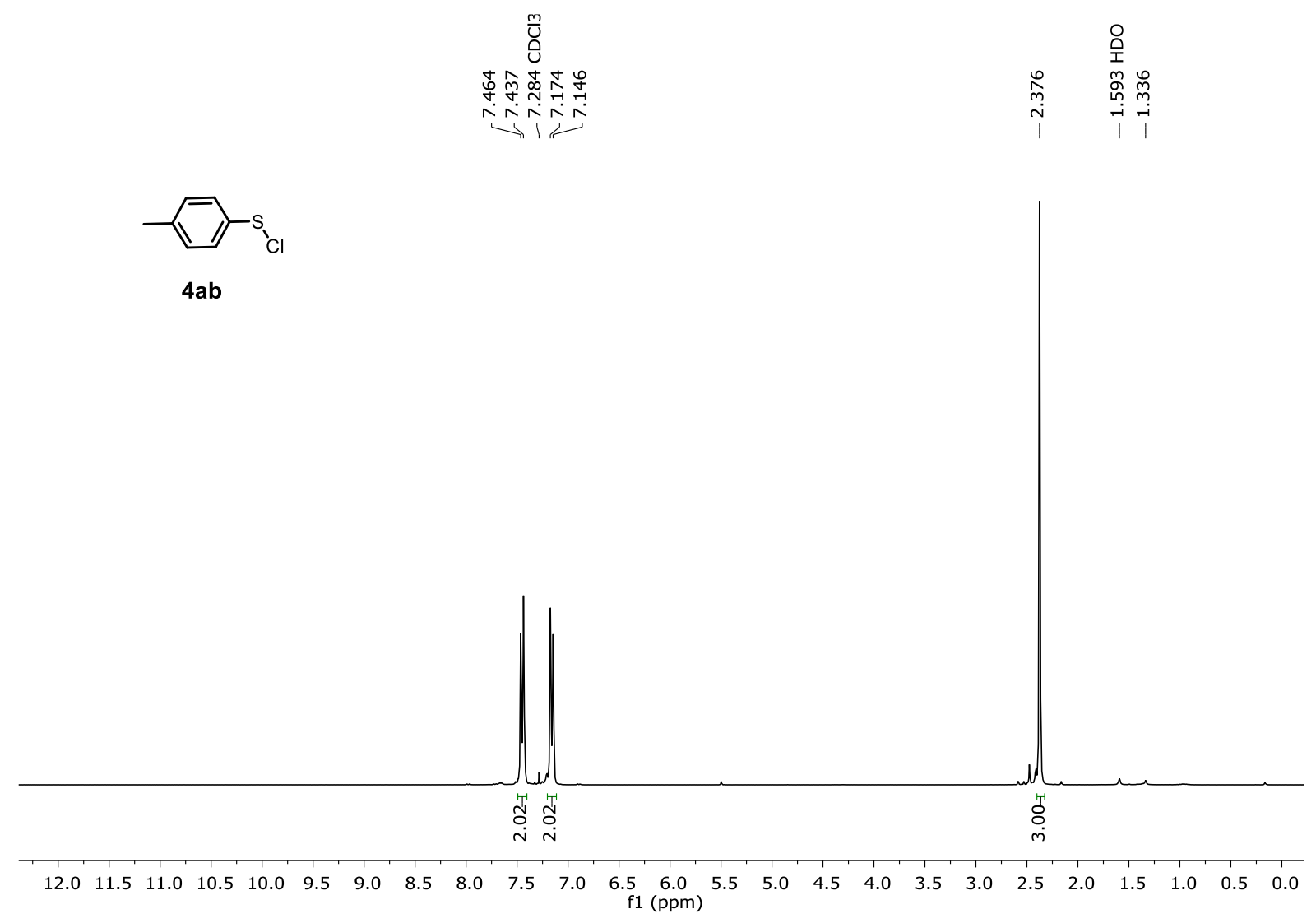

Figure S95: ${ }^{1} \mathrm{H}$ NMR spectrum of compound 4ab in $\mathrm{CDCl}_{3}(300 \mathrm{MHz})$

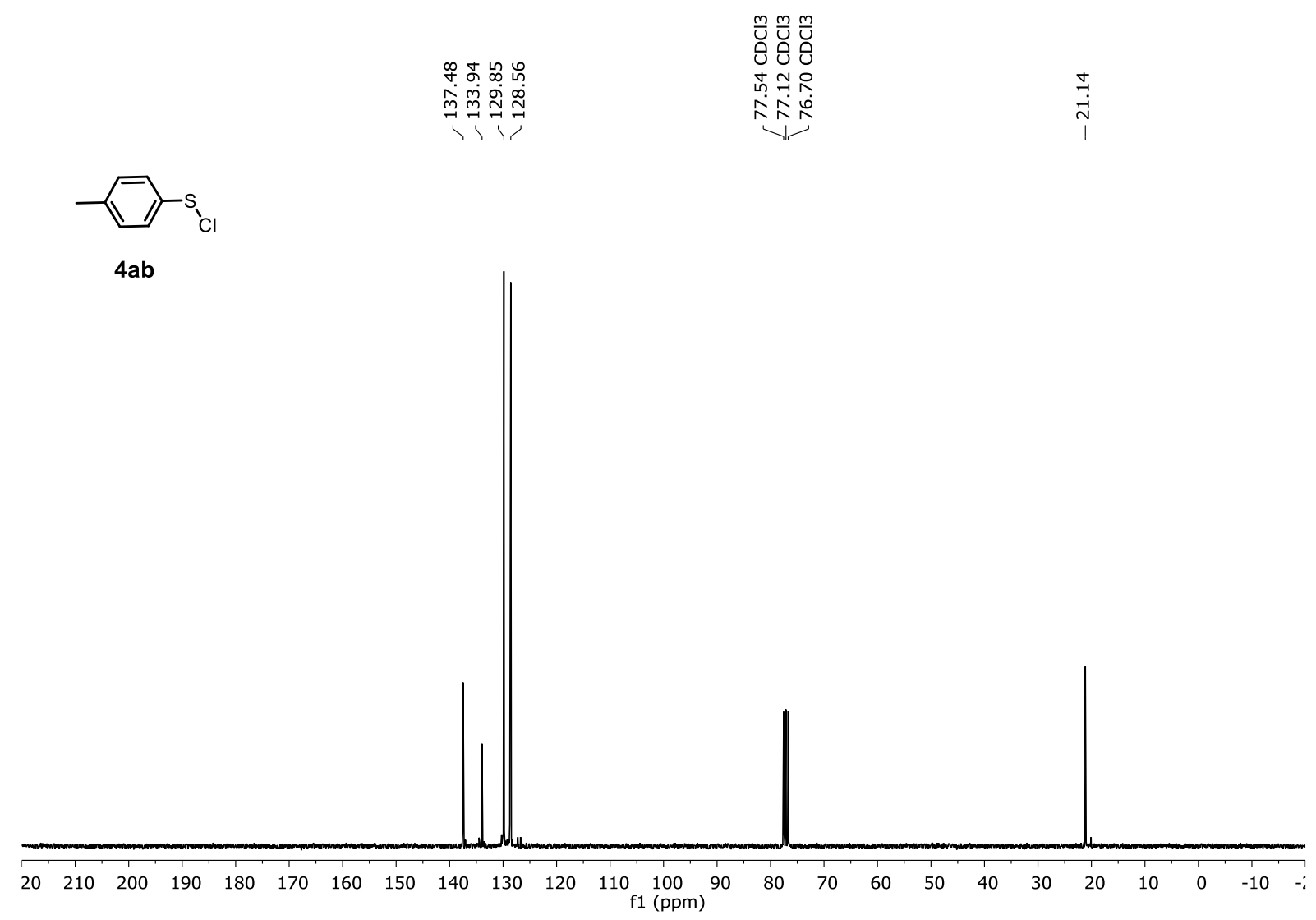

Figure S96: ${ }^{13} \mathrm{C}\left\{{ }^{1} \mathrm{H}\right\}$ NMR spectrum of compound $\mathbf{4 a b}$ in $\mathrm{CDCl}_{3}(75 \mathrm{MHz})$ 


\section{Intermediate I}

30-Dec-2020

30DEC_2020_LCMS_YMC_FA+ACN_34

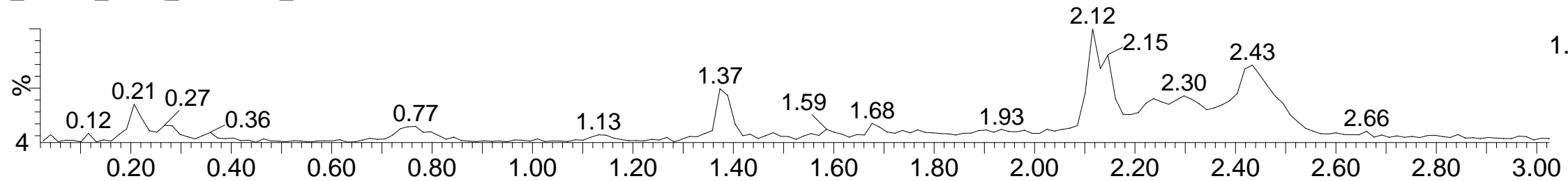

30DEC_2020_LCMS_YMC_FA+ACN_34

$$
100
$$
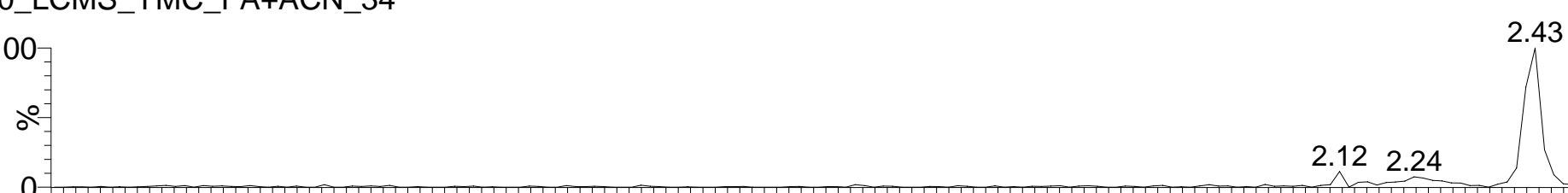

1: Scan ES+
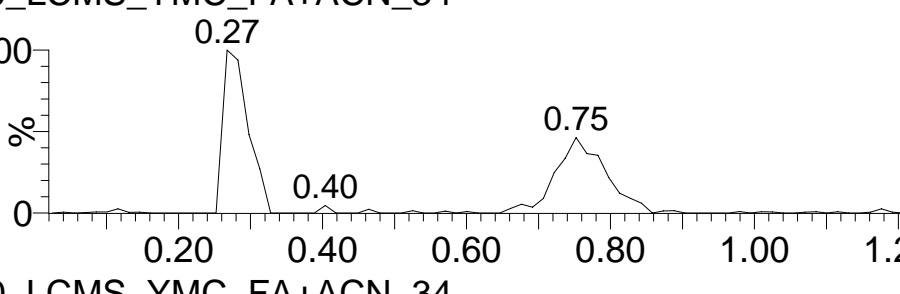

$\begin{array}{lll}0.20 & 0.40 & 0.60 \\ Y M C & & 0.40\end{array}$

30DEC_2020_
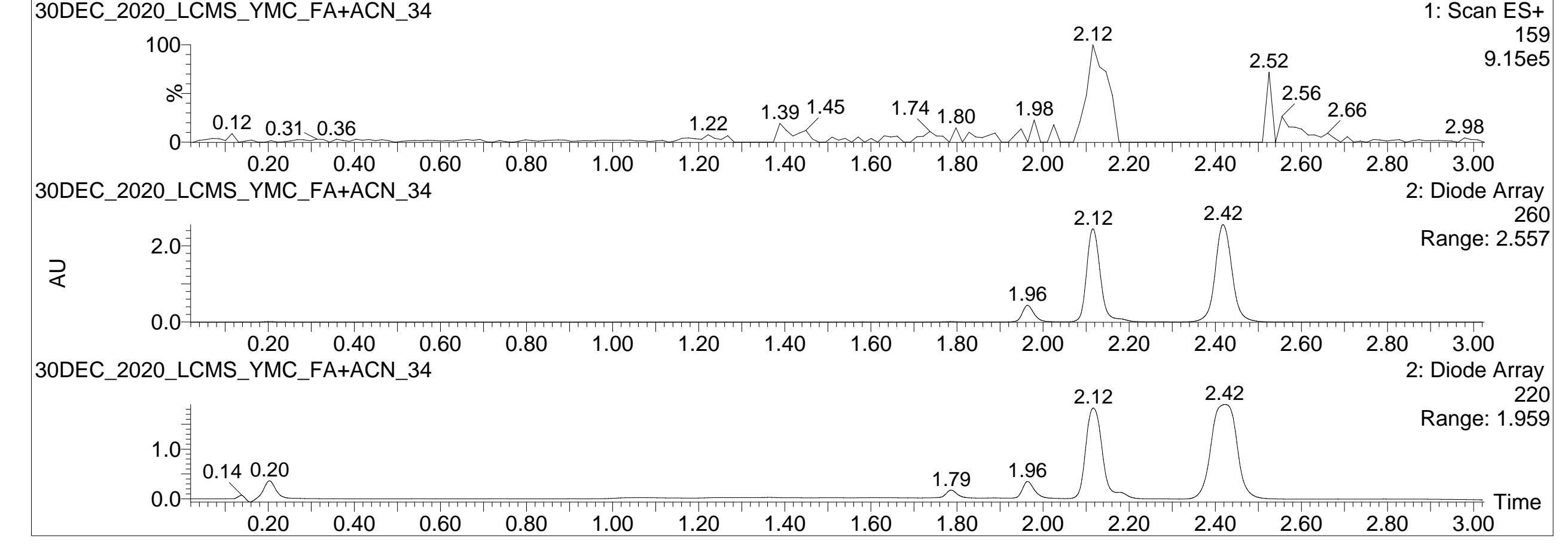

$\begin{array}{lll}1.00 & 1.20 & 1.4\end{array}$
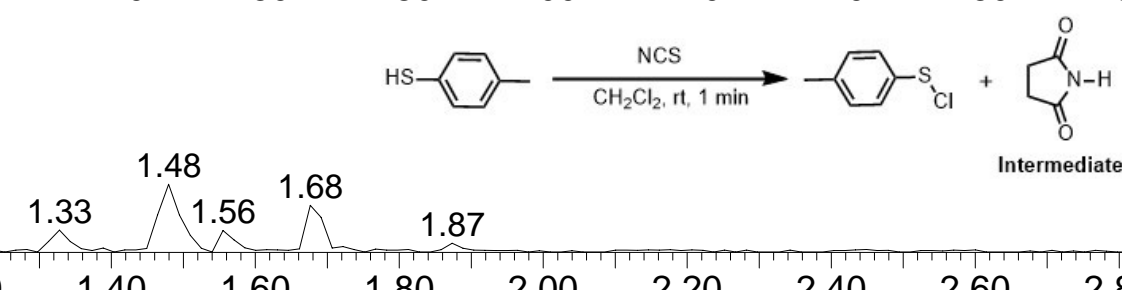

Scan ES+ 101
$7.13 \mathrm{e} 6$ Intermediate I $4.03 \mathrm{e} 5$ TIC
$1.45 \mathrm{e} 8$ 


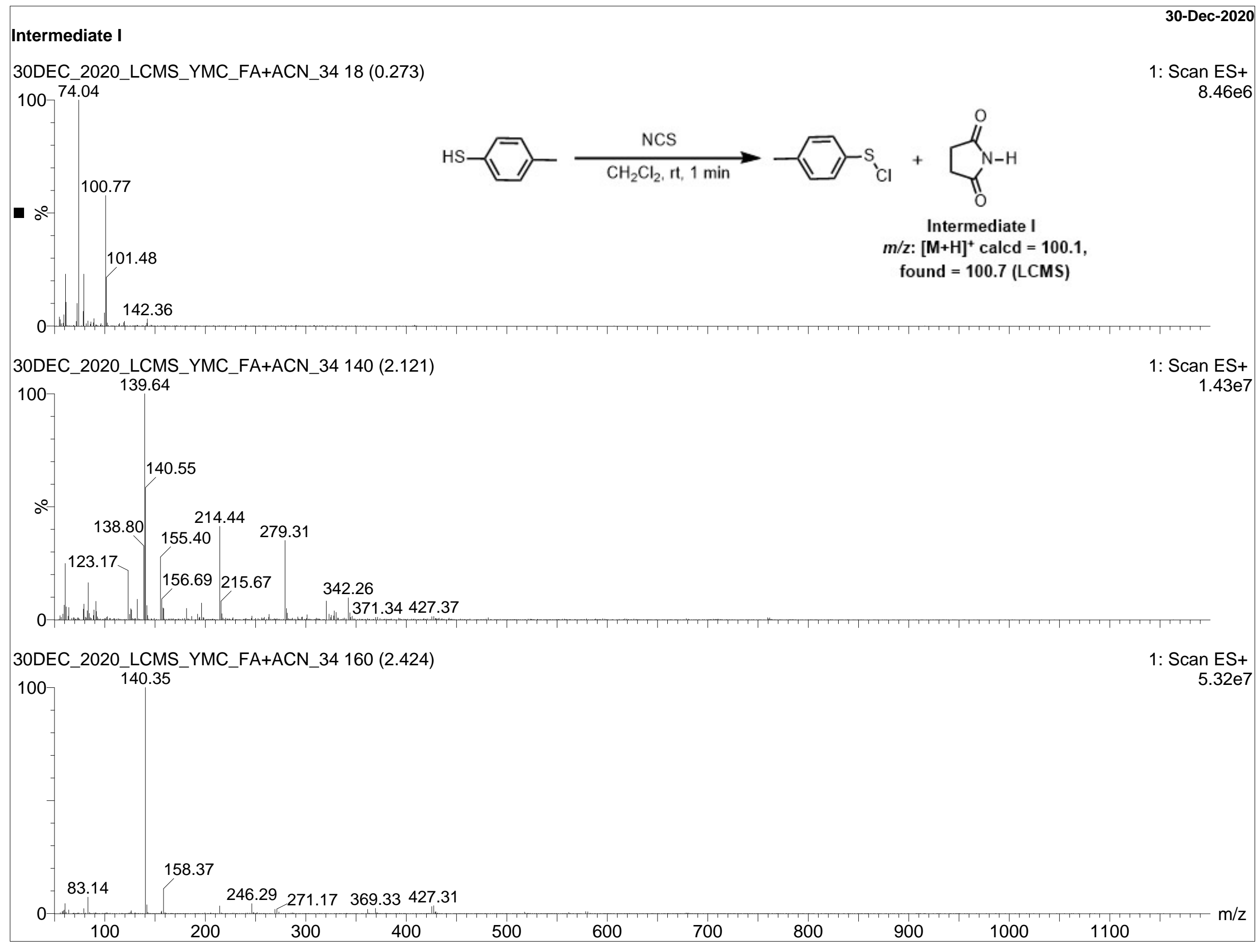

Figure S98: LC-MS Mass spectrum of Intermediate I 